\title{
Characterization of
}

\section{Mitochondrial Calcium Uniporter in Barth Syndrome Models}

\author{
Dissertation \\ for the award of the degree \\ "Doctor rerum naturalium" \\ of the Georg-August-Universität Göttingen
}

within the doctoral program

"Göttingen Graduate Center for Neurosciences, Biophysics and Molecular Biosciences (GGNB) - Molecular Biology of Cells" of the Göttingen Center for Molecular Biosciences (GZMB)

\author{
submitted by \\ Magnus Hartmann
}

from Hildesheim, Germany

Göttingen, 2020 


\section{Members of the Thesis Committee and Examination Board}

Prof. Dr. Peter Rehling

(Supervisor and first referee)

Prof. Dr. Doerthe Katschinski

(Second referee)

Prof. Dr. Blanche Schwappach
Department of Cellular Biochemistry

University Medical Center Göttingen

Göttingen, Germany

Department of Cardiovascular Physiology

Heart Center Göttingen

Göttingen, Germany

Department of Molecular Biology

University Medical Center Göttingen

Göttingen, Germany

\section{Further members of the Examination Board}

Prof. Dr. Ralph Kehlenbach

Prof. Dr. Detlef Doenecke

Prof. Dr. Henning Urlaub
Department of Molecular Biology

University Medical Center Göttingen

Göttingen, Germany

Department of Molecular Biology

University Medical Center Göttingen

Göttingen, Germany

Max Planck Institute for Biophysical Chemistry

Göttingen, Germany 


\section{Affidavit}

Hereby I declare, that my dissertation "Characterization of Mitochondrial Calcium Uniporter in Barth Syndrome Models" has been prepared independently. No more assistence and sources than quoted has been used.

Magnus Hartmann

Göttingen, 2020 


\section{Table of Contents}

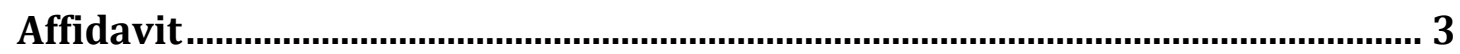

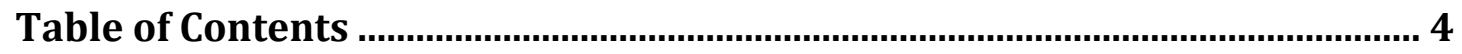

Table of figures ...........................................................Error! Bookmark not defined.

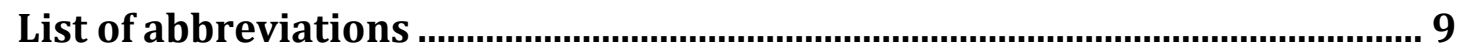

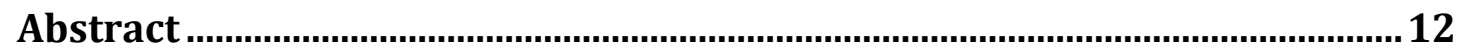

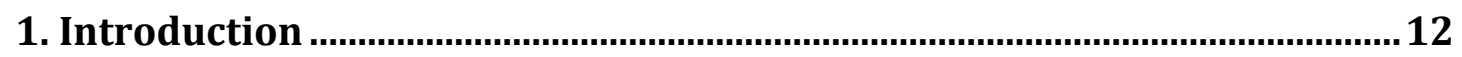

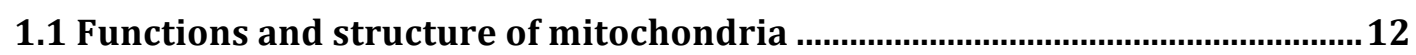

1.2 Cardiolipin: a crucial phospholipid for structural integrity and function of

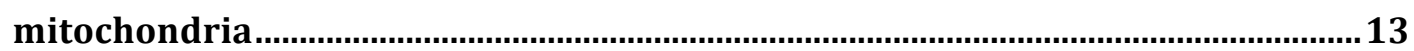

1.3 Cardiolipin - biosynthesis, remodeling and degradation ................................ 14

1.3.1 Cardiolipin biosynthesis ................................................................................................14

1.3.2 Cardiolipin remodeling........................................................................................16

1.3.3 Tafazzin - the key enzyme in cardiolipin remodeling .............................................17

1.3.4 The role of MLCLAT1 and ALCAT1 in cardiolipin remodeling.............................18

1.3.5 Cardiolipin degradation .........................................................................................18

1.4 Role of cardiolipin in mitochondrial functions .................................................... 19

1.4.1 Mitochondria-targeted protein translocation via TOM and TIM23 complex...19

1.4.2 Cardiolipin and its involvement in oxidative phosphorylation ...........................20

1.4.3 Role of cardiolipin: Mitophagy, apoptosis and morphology maintenance........22

1.5 Barth syndrome (BTHS) - Pathophysiology of tafazzin deficiency and

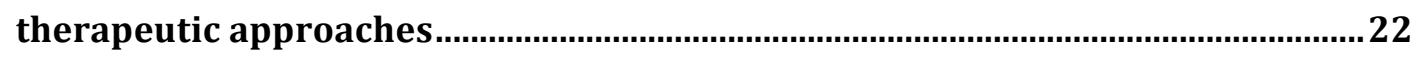

1.6 The role of mitochondria in calcium metabolism..........................................24

1.7 The Mitochondrial Calcium Uniporter (MCU) complex ..................................25

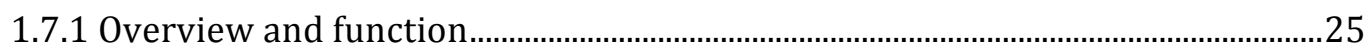

1.7.2 Mitochondrial Calcium Uniporter (MCU) protein ..................................................26

1.7.3 Mitochondrial Calcium Uptake 1 protein (MICU1).................................................27

1.7.4 Mitochondrial calcium uniporter regulator 1 (MCUR1) ........................................28

1.7.5 Mitochondrial Calcium Uniporter subunit b (MCUb) ............................................22

1.7.6 EMRE, the Essential MCU Regulator ………………..................................................29

1.7.7 Proteolytic regulation of MCU composition and activity .......................................30

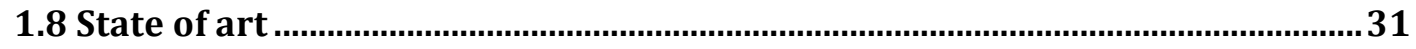

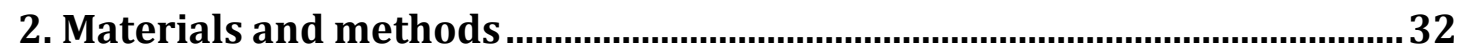


2.1.1 Chemicals

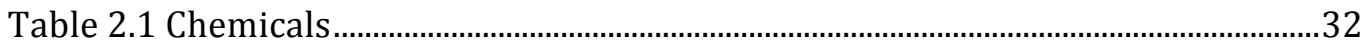

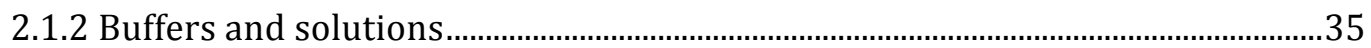

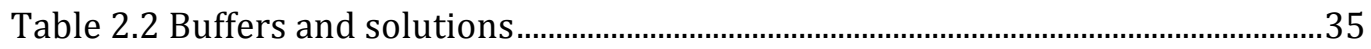

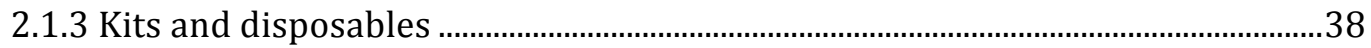

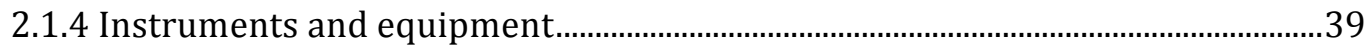

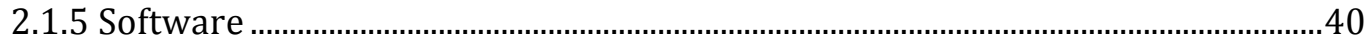

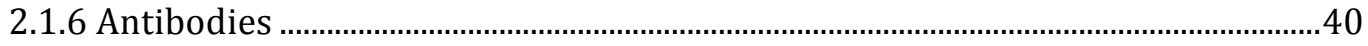

2.1.7 Oligonucleotides and plasmids ........................................................................... 41

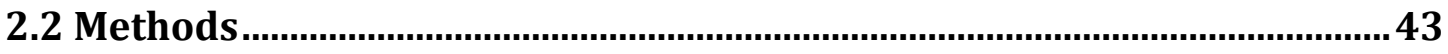

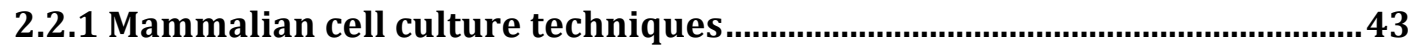

2.2.1.1 Cultivation of Mouse Embryonic Fibroblasts (MEF cells) ..................................4

2.2.1.2 Silencing of mRNA/protein expression levels via RNAi .....................................4

2.2.1.3 Electroporation of plasmid DNA............................................................................4

2.2.1.4 Control and patient-derived inducent pluripotent stem cell cardiomyocytes

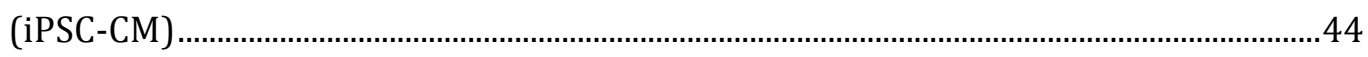

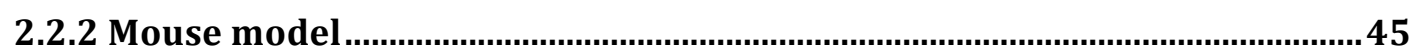

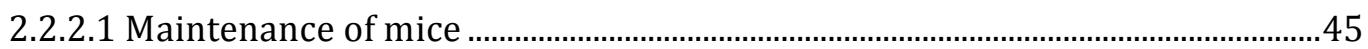

2.2.2.2 Mitochondria isolation from mouse tissues via differential centrifugation .45

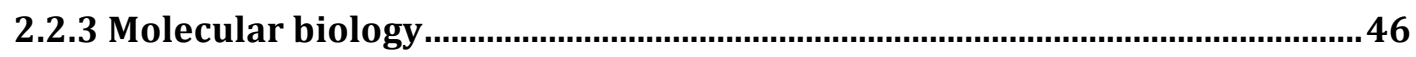

2.2.3.1 Transformation of plasmid into E.coli ………....................................................... 46

2.2.3.2 Plasmid DNA isolation from E.coli.........................................................................46

2.2.3.3 Polymerase chain reaction (PCR) .......................................................................

2.2.3.4 Agarose gel electrophoresis................................................................................4

2.2.3.5 Purification of PCR products …………………..................................................... 48

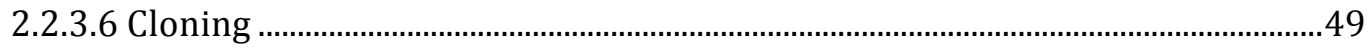

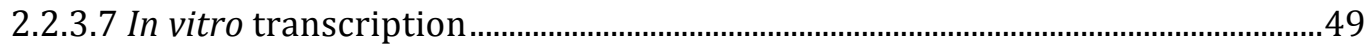

2.2.3.8 In vitro translation ..............................................................................................50

2.2.4 Protein separation and protein complex analysis ............................................50

2.2.4.1 Determination of protein concentration via Bradford assay .............................50

2.2.4.2 Protein separation via SDS-PAGE analysis ..........................................................51

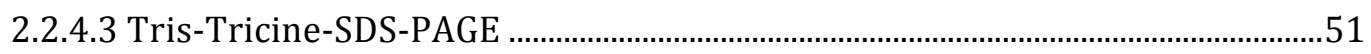

2.2.4.4 Blue Native Polyacrylamid Gel Electrophoresis (BN-PAGE) .............................52

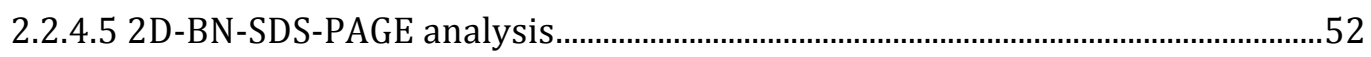

2.2.4.6 Western Blotting and Immunodetection................................................................53 
2.2.5.1 Crude isolation of mitochondrial fractions from mammalian cells via differential centrifugation

2.2.5.2 Carbonate extraction .55

2.2.5.3 Submitochondrial localization studies by swelling of mitochondria. .55

2.2.5.4 FLAG immunoprecipitation ................................................................................56

2.2.5.5 Antibody coating to Protein-A-Sepharose (PAS) ……………………………......57

2.2.5.6 Co-immunoprecipitation (Co-IP) with antibodies coated to PAS.......................57

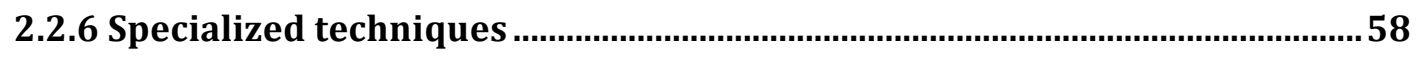

2.2.6.1 Radiolabeled in-vitro preprotein import into mitochondria..............................58

2.2.6.2 Lipid extraction and Thin Layer Chromatography (TLC) ...................................59

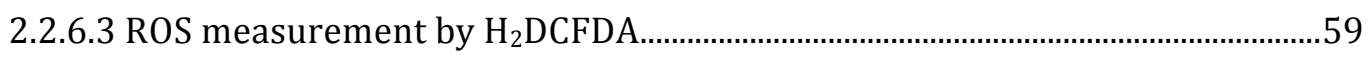

2.2.6.4 Measurements of mitochondrial calcium uptake ................................................6

\section{Results .......................................................................................................60}

3.1 Characterisation of MCU constituents in WT and TAZ KO MEF cells ...............61

3.1.1 Steady state levels of mitochondrial proteins ......................................................61

3.1.2 Mitochondrial localization of core constituents of MCU ......................................62

3.2 Analysis of MCU complex composition and organization in TAZ KO MEF cells

64

3.2.1 2D-BN-SDS-PAGE analysis of MCU/EMRE-containing complexes in MEF cells

3.2.2 EMRE-containing complexes interact with MCU oligomers...................................66

3.2.3 Verification of MCU signal specificity by MCU RNA silencing...............................67

3.2.4 The interaction of MCU and EMRE is not affected in TAZ KO MEF

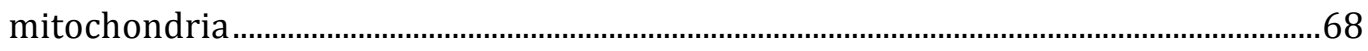

3.2.5 MICU1 pulldown via depletion IP impact MCU MDa complex...............................70

3.2.6 Complementation of TAZ KO MEF cells by exogenous tafazzin expression:....72

3.2.7 MCU complex dissociation and respiratory chain remodeling in TAZ KO MEF mitochondria are rescued by tafazzin complementation ................................................73

3.2.8 MCU complex stability in MEF cells is dependent on appropriate cardiolipin biosynthesis, but not reactive oxygen species (ROS) .......................................................75

3.2.9 Quantification of MCU complex ratio shift in WT and TAZ KO MEF cells..........76

3.3 Mitochondrial calcium uptake is affected in TAZ KO cells...............................77

3.4 Import and assembly of MCU and EMRE in WT/TAZ KO MEF mitochondria 79

3.4.1 Tafazzin deficiency does not affect translocases in MEF cells 
3.4.2 MCU and EMRE are presequence-containing mitochondrial proteins

3.4.3 Import of radiolabeled EMRE and motor-dependent import of OTC into MEF mitochondria is not affected by TAZ KO

3.4.4 Interaction of newly imported radioactive EMRE with MCU-FLAG is not affected in TAZ KO MEF mitochondria.

3.4.5 In-vitro precursor import of radiolabeled MCU into isolated WT and TAZ KO MEF mitochondria

3.4.6 EMRE interaction with imported MCU is not affected in TAZ KO MEF cell mitochondria

3.4.7 Turnover of newly imported MCU is unchanged in WT and TAZ KO MEF mitochondria

3.4.8 Insertion of proteins containing 2 transmembrane domains (TMDs) is not affected in TAZ KO

3.4.9 Lack of MICU1 does not affect MCU in-vitro import

3.4.10 Membrane potential sensitivity of MCU import in TAZ KO mitochondria .....89

3.5 MCU complexes in different Barth syndrome mouse organs. 90

3.5.1 MCU complexes are tissue-specifically organized

3.5.2 Protein steady state levels of respiratory chain components and MCU in mouse skeletal muscle

3.5.3 MCU/EMRE interaction in BTHS mouse skeletal muscle is unaffected. .93

3.5.4 MCU and respiratory chain complexes in BTHS mouse brain are dissociated 96 3.5.5 Tafazzin-deficiency does not affect MCU complex organization in BTHS mouse liver.

3.5.6.2 Steady state levels of MCU complexes are reduced in mitochondria of BTHS mouse hearts and BTHS patient derived cardiomyocytes 100

4. Discussion 102

4.1 MCU complex organization in BTHS models. 102

4.2. Increased basal mitochondrial calcium uptake and the role of MICU1 in TAZ KO MEF cells. 103

4.3 MCU complex formation and role of reactive oxygen species (ROS) 105

4.4 Role of cardiolipin on MCU and respiratory chain complexes in cardiac tissues

4.5 Mitochondrial import and stability of MCU precursor protein 109

5. Conclusion of results 
6.1 MCU reduction in BTHS cardiac mitochondria ............................................... 111

6.2 MCU interaction partners and complex composition......................................... 112

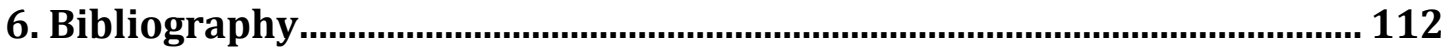

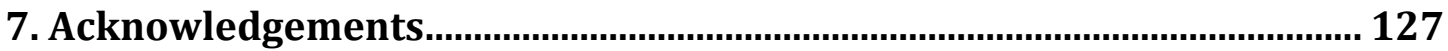

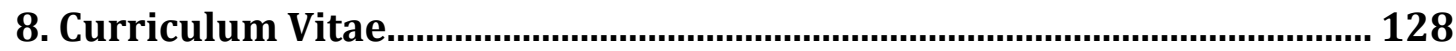

List of tables

List of contents 


\section{List of abbreviations}

$\begin{array}{ll}\text { ADP } & \text { Adenosine diphosphate } \\ \text { APS } & \text { Ammonium persulfate } \\ \text { ATP } & \text { Adenosine triphosphate } \\ \text { AVO } & \text { Antimycin A, valinomycin, oligomycin mixture } \\ \text { BN } & \text { Blue native } \\ \text { bp } & \text { Base pair(s) } \\ \text { BSA } & \text { Bovine serum albumin } \\ { }^{\circ} \text { C } & \text { Degree Celsius } \\ \text { CIII } & \text { Complex III } \\ \text { CL } & \text { Cardiolipin } \\ \text { COX/CIV } & \text { Cytochrome } c \text { oxidase/ complex IV } \\ \text { Cys } & \text { Cysteine } \\ \text { DMSO } & \text { Dimethyl sulfoxide } \\ \text { DNA } & \text { Deoxyribonucleic acid } \\ \text { ECL } & \text { Enhanced chemiluminescence } \\ \text { EDTA } & \text { Ethylenediaminetetraacetic acid } \\ \text { EGTA } & \text { Ethyleneglycoltetraacetic acid } \\ \text { EMRE } & \text { Essential MCU regulator } \\ \text { FCS } & \text { Fetal calf serum } \\ \text { g } & \text { gram } \\ \text { HEPES } & \text { 4-(2-hydroxyethyl)-1-piperazineethanesulfonic acid } \\ \text { HRP } & \text { Horseradish peroxidase } \\ \text { IgG } & \text { Immunoglobulin G } \\ \text { IMM } & \text { Inner mitochondrial membrane } \\ \text { IMS } & \text { Intermembrane space } \\ \text { kDa } & \text { Kilodalton } \\ \text { KD } & \text { Knockdown } \\ & \end{array}$




\begin{tabular}{|c|c|}
\hline $\mathrm{KO}$ & Knockout \\
\hline 1 & Liter \\
\hline LB & Lysogeny broth \\
\hline $\mathrm{m}$ & Mature protein \\
\hline M & Moles per liter \\
\hline $\mathrm{mA}$ & Milliampere \\
\hline $\mathrm{MCU}$ & Mitochondrial Calcium Uniporter \\
\hline MCUb & Mitochondrial Calcium Uniporter subunit b \\
\hline MCUR1 & Mitochondrial Calcium Uniporter regulator \\
\hline $\mathrm{MDa}$ & Megadalton \\
\hline MICU & Mitochondrial calcium uptake \\
\hline $\mathrm{mg}$ & Milligram \\
\hline MIA & Mitochondrial IMS assembly machinery \\
\hline MICOS & Mitochondrial contact site and cristae organizing system \\
\hline $\min$ & Minute(s) \\
\hline mito & Mitochondria \\
\hline $\mathrm{mM}$ & Millimoles per liter \\
\hline MOPS & 3-(N-morpholino)propanesulfonic acid \\
\hline mRNA & Messenger RNA \\
\hline NADH & Nicotinamide adenine dinucleotide \\
\hline $\mathrm{nm}$ & Nanometer \\
\hline NT & Non-targeting \\
\hline OD & Optical density \\
\hline OMM & Outer mitochondrial membrane \\
\hline ORF & Open reading frame \\
\hline OXPHOS & Oxidative phosphorylation \\
\hline $\mathrm{p}$ & precursor \\
\hline PAGE & Polyacrylamide gel electrophoresis \\
\hline PAM & Presequence translocase-associated motor \\
\hline PBS & Phosphate buffered saline \\
\hline $\mathrm{PC}$ & Phosphatidylcholine \\
\hline
\end{tabular}


PCR Polymerase chain reaction

PE Phosphatidylethanolamine

PG Phosphatidylglycerol

PK Proteinase $\mathrm{K}$

PMSF Phenylmethylsulfonyl fluoride

PVDF Polyvinylidene fluoride

RNA Ribonucleic acid

ROS Reactive oxygen species

rpm Revolutions per minute

SDH Succinate dehydrogenase

SEM Standard error of the mean

SDS Sodium dodecyl sulfate

SILAC Stable isotope labeling of amino acids in cell culture

siRNA Small interfering RNA

TBS Tris buffered saline

TCA Trichloroacetic acid

TEMED N,N,N',N'-Tetramethylethane-1,2-diamine

TIM22 Carrier translocase of the inner membrane

TIM23 Presequence translocase of the inner membrane

TLC Thin-layer chromatography

TMD Transmembrane domain

TOM Translocase of the outer membrane

Tris Tris(hydroxymethyl)aminomethane

UV Ultraviolet

V Volt(s)

WT Wild-type

$\mu 1 \quad$ Microliter(s)

$\mu \mathrm{g} \quad$ Microgram(s) 


\section{Abstract}

Fdf

\section{Introduction}

\subsection{Functions and structure of mitochondria}

Mitochondria are organelles of eukaryotic cells, which are known for their ability to convert chemical energy of lipids, amino acids and monosaccharides to a metabolically usable form by oxidative phosphorylation (OXPHOS). Besides the function of generating adenosine triphosphate (ATP) by respiration, mitochondria fulfill various metabolic functions, such as the metabolism of lipids and amino acids, formation of iron-sulfur clusters and heme, urea cycle and calcium homeostasis (Dudek et al., 2019; Friedman et al., 2011; Lill and Mühlenhoff, 2008). The exertion of all mitochondrial functions requires a conserved compartmentation by inner mitochondrial membrane (IMM) and outer mitochondrial membranes (OMM). The OMM connects mitochondria to other cell organelles, such as plasma membrane, lysosome, peroxisome, lipid droplets and endo- or sarcoplasmic reticulum (ER) via formation of contact sites (Elbaz-Alon et al., 2014). These contact sites facilitate intracellular communication and an exchange of lipids and small solute molecules (Tatsuta et al., 2016). Furthermore, mitochondria are partitioned in two compartments, the mitochondrial matrix and the intermembrane space (IMS), separated by the IMM. The IMM presents in two different morphological shapes. It forms invaginations, called cristae, where OXPHOS protein complexes reside. Additionally, the inner boundary membrane, which is in proximity to the OMM, localizes the translocases of the protein import machineries (Dudek et al., 2018). The compartmentation by the IMM allows the generation of a membrane potential as the crucial driving force for all mitochondrial functions (ATP production, mitochondrial 
protein import and biogenesis, etc.). The specific morphology of IMM as well as the well-orchestrated functional diversity of mitochondria requires the special phospholipid composing mitochondrial membranes known as cardiolipin. This dimeric phospholipid resides specifically in inner mitochondrial membrane and is involved in various mitochondrial functions, which are introduced in the following chapters.

\subsection{Cardiolipin: a crucial phospholipid for structural integrity and function of mitochondria}

The structural and functional role of lipids in biological membranes in eukaryotic cells has been extensively studied within the last decades. However, the composition of mitochondrial membranes and their crucial role for mitochondrial functions received less attention (Saric et al., 2016). Meanwhile, the role of phospholipids in mitochondrial membranes were highlighted in signaling pathways in connection with mitophagy, apoptosis and cell cycle regulation. The participation of membranes in cellular processes requires special properties including fluidity, lateral mobility and curvature maintenance (Kagan et al., 2014). In general, biological membranes in eukaryotic cells are composed of amphiphilic phospholipid bilayers. The head is the hydrophilic phosphate group esterified with two hydrophobic acyl chains (ColinaTenorio et al., 2020; Kagan et al., 2014). Mitochondrial membranes reveal differences in their phospholipid composition in comparison to other organelle membranes, enabling the crucial mitochondrial functions. In fact, the phospholipid-to-protein ratio is reduced compared to other cell organelles. The phosphatidylethanolamine (PE) and phosphatidylcholine (PC) content is exceptionally high in mitochondrial membranes and both phospholipids account for $80 \%$ of the total membrane phospholipid pool (Saric et al., 2016). Additional properties of mitochondrial membranes are the low levels of sphingolipids and sterols, but one particular phospholipid shows a strong enrichment in mitochondria and is indispensable for its function: Bis-(1,2-diacyl-snglycero-3-phospho)-1'-3'-sn-glycerol or cardiolipin, which is the hallmark polyglycerophospholipid in mitochondrial membranes in mammalian cells (Horvath and Daum, 2013). Cardiolipin owes its name from the first isolation from beef heart (Pangborn, 1946) and its biosynthesis pathway in mammalian cells was elucidated in 
rat liver (Hostetler et al., 1971). Cardiolipin was particularly found in the inner mitochondrial membrane and lesser in the outer mitochondrial membrane (Hovius et al., 1990, 1993). It has also been observed in mitochondrial contact sites (MCS), where the outer and inner mitochondrial membrane come to a proximal contact enabling variable functions in the regulation of cellular trafficking, apoptosis, immune response and organelle dynamics (Nicolay et al., 1990). Cardiolipin contains a glycerol backbone connected with two phosphatidylglycerols, forming a dimeric structure (Dudek et al., 2016; Hoch et al., 1992; Maguire et al., 2016). Each phosphatidylglycerol is esterified with two acyl chains, hence, the hydrophobic tail is composed of four acyl chains. The acyl chains in completely remodeled cardiolipin are unsaturated. The variety of cardiolipin species is reasoned by the diversity of mono- and polyunsaturated fatty acids in eukaryotic cells. In fact, bacterial and eukaryotic cardiolipins differ in two aspects. The acyl chains of cardiolipin differ in the degree of saturation and the chain length. Eukaryotic cardiolipins are polyunsaturated and longer (18-22 carbon atoms) than cardiolipins from prokaryotes, which are saturated or mono-unsaturated and have a shorter chain length. These differences were caused by the adaption from anaerobic to aerobic metabolism in the evolution from prokaryotes to eukaryotic cells. The double bonds of unsaturated acyl chains were oxidized by molecular oxygen, with the result that cardiolipin in mitochondria participates in signaling pathways (Saric et al., 2016; Tyurina et al., 2014). The predominant form in the mammalian heart is tetra-linoleic acid (Hoch et al., 1992). Impairments in the processes of cardiolipin biogenesis and remodeling cause severe pathophysiological disorders like Barth syndrome (Dudek et al., 2016, 2018).

To understand the unique role of cardiolipin in mitochondrial functions compared to the other phospholipids, it is worth considering the biogenesis and remodeling process of cardiolipin.

\subsection{Cardiolipin - biosynthesis, remodeling and degradation}

\subsubsection{Cardiolipin biosynthesis}

The majority of the phospholipids phosphatidylethanolamine (PE), phosphatidylserine 
(PS) and phosphatidic acid (PA) are synthesized in the endoplasmatic reticulum (ER) (Hatch et al., 2004; Horvath and Daum, 2013). In contrast, cardiolipin is exclusively synthesized in the inner mitochondrial membrane (Kagan et al., 2014; Mejia et al., 2014).

Newly synthesized phospholipids can be transported to mitochondrial membranes serving as precursors for membrane formation and precursors of cardiolipin biosynthesis (Connerth et al., 2012; Tamura et al., 2009). Cardiolipin biosynthesis in mitochondrial membranes is evolutionary conserved in eukaryotic cells (Schlame and Halder, 1993).

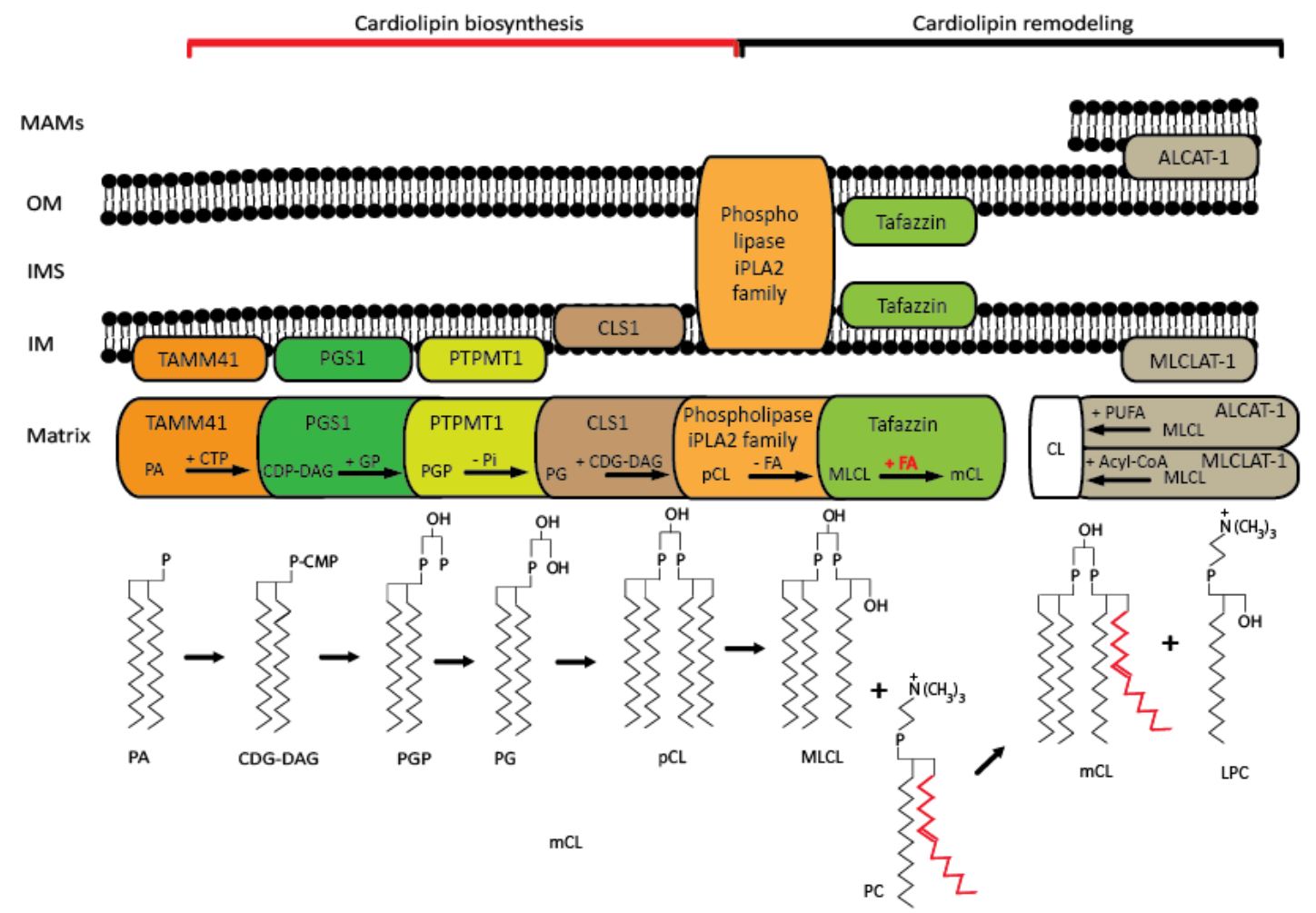

Figure 1.1: The different steps of cardiolipin biosynthesis and remodeling (Dudek et al., 2018). Cardiolipin biosynthesis takes place exclusively in mitochondrial membranes. Newly synthetized cardiolipin needs to undergo a remodeling process enabled mainly by the transacylase tafazzin, but MLCLA1 and ALCAT1 as well. IM: inner membrane, OM: outer membrane, IMS: intermembrane space, PUFA: poly unsaturated fatty acids, pCL: precardiolipin, mCL: mature cardiolipin

Enzymes involved in cardiolipin biosynthesis pathway are located in the inner leaflet of the inner mitochondrial membrane, but cardiolipin is found in the outer leaflet of the inner membrane and the other mitochondrial membrane as well (Figure 1.1) (Saric 
et al., 2016). The first catalytic step of cardiolipin biosynthesis is similar to phospholipid synthesis in ER: the conversion of PA. PA is the direct precursor of cardiolipin synthesis. In the first reaction, PA is converted into cytidine diphosphatediacylglycerol (CDP-DAG) and pyrophosphate, accompanied by the addition of cytidine triphosphate (CTP). This reaction is catalysed by CDP-DAG synthase (CDS) (Kuchler et al., 1986; Shen et al., 1996). The mitochondria-resident CDS is called TAMM41 and initiates the cardiolipin biosynthesis (Kutik et al., 2008; Tamura et al., 2006; Tamura and Endo, 2013). CDP-DAG and glycerol phosphate (GP) are the substrates of the second reaction step catalyzed by the phosphatidylglycerol phosphate synthase (PGS1), forming phosphatidylglycerol phosphate (PGP) and cytidine monophosphate (CMP) (Chang et al., 1998). PGS1 activity is regulated by phosphorylation and may adapt to metabolic changes and mitochondrial biogenesis in yeast (Shen et al., 1998; Zhong et al., 2003). PGP is dephophorylated to phosphatidylglycerol (PG) by protein-tyrosine phosphatase mitochondrial 1 (PTPMT1) (Xiao et al., 2011; Zhang et al., 2011). The final step of cardiolipin biosynthesis is realized by the cardiolipin synthase (CLS) (Chang et al., 1998; Chen et al., 2006; Lu et al., 2006), an integral inner membrane protein, which faces the matrix site (Schlame and Halder, 1993). The underlying reaction is condensation of CDPDAG with PG, generating cardiolipin in this step. Interestingly, PGS1 and CLS tend to assemble to a hetero-oligomeric complex which is associated to the inner mitochondrial membrane by interacting with cardiolipin-binding proteins like the MICOS complex, stomatin-like protein 2 and prohibitins (Serricchio et al., 2018).

\subsubsection{Cardiolipin remodeling}

The CLS reaction forms immature cardiolipin, which requires to be remodeled by an exchange of saturated acyl chains with unsaturated acyl chains (Chang et al., 1998; Chen et al., 2006; Lu et al., 2006). The deacylation step to form monolysocardiolipin (MLCL) is suggested to be performed by calcium-independent phospholipases (iPLA 2 family) (Figure 1.1) (Boynton et al., 2015; Mancuso et al., 2007; Malhotra et al., 2009; Yoda et al., 2010). Newly synthetized cardiolipin is characterized by an asymmetric central carbon atom of the esterified glycerol and contains saturated acyl chains of different lengths (Schlame et al., 2005; Schlame and Ren, 2006), whereas mature cardiolipin is characterized by symmetric unsaturated acyl chains where all 
acyl chains contain the same length and degree of saturation (Claypool and Koehler, 2012; Schlame et al., 2005).

Subsequently, the three different mitochondrial enzymes MLCLAT1, ALCAT1 and tafazzin are known to be capable of remodeling immature cardiolipin to the mature form.

\subsubsection{Tafazzin - the key enzyme in cardiolipin remodeling}

Tafazzin, a phospholipid-lysophospholipid transacylase, resides in the inner mitochondrial membrane as well as in the inner leaflet of the inner membrane (Brandner et al., 2005; Claypool et al., 2006; Gebert et al., 2009; Xu et al., 2015). It catalyzes the transfer of an unsaturated acyl chain from phospholipid to lysophospholipid (Saric et al., 2016). It is ubiquitously expressed and highly conserved across species. Tafazzin is encoded by the TAZ gene (TAZ, G4.5, OMIM 300394) and is named after an Italian comic character ("Tafazzi”). Deficiency of tafazzin causes a decrease in remodeled cardiolipin and an increased monolysocardiolipin-to-cardiolipin ratio, which serves as a diagnostic marker for Barth syndrome (Houtkooper et al., 2009). Tafazzin activity shows substrate specificity towards cardiolipin and phosphatidylcholin. One acyl chain hydrolysed from phosphatidylcholine/lecithin (PC) is esterified with monolysocardiolipin by tafazzin ( $\mathrm{Xu}$ et al., 2009). The substrate specificity could be explained by the properties of proximal lipid domains where tafazzin is embedded (Schlame et al., 2012). However, the substrate specificity does not refer to the various acyl chains, which can undergo transacylation. Tafazzin can act at the $s n-1$ and also at $s n-2$ positions on acyl chains (Lu et al., 2006; Malhotra et al., 2009). This leads to a high variety of cardiolipin species maturated by tafazzin in different organs. Hence, tafazzin deficiency like in Barth syndrome causes tissue specific alterations in cardiolipin pools (Cole et al., 2016; Dudek et al., 2016; Peyta et al., 2016). Furthermore, tafazzin activity requires a lipid environment, which is formed by the cochaperone DnaJ Heat Shock Protein Family Member C19 (DNAJC19) and cardiolipin-interacting scaffold proteins, called prohibitins (Richter-Dennerlein et al., 2014). 


\subsubsection{The role of MLCLAT1 and ALCAT1 in cardiolipin remodeling}

The coenzyme A-dependent monolysocardiolipin acyltransferase (MLCLAT1) was characterized as a linoleoyl specific transferase (Taylor et al., 2009). MLCLAT1 resides in the inner leaflet of the inner mitochondrial membrane (Figure 1.1) (Taylor et al., 2012). Silencing of MLCLAT1 in Hela cells led to a decreased incorporation of linoleic acid into cardiolipin (Taylor et al., 2012), whereas overexpression of MLCLAT1 extended reacylation of monolysocardiolipin in Barth Syndrome lymphoblasts and led to higher incorporation of linoleic acid into cardiolipin (Mejia et al., 2018; Taylor et al., 2012).

The third known enzyme involved in cardiolipin maturation is acylCoA:lysocardiolipin acyltransferase 1 (ALCAT1). ALCAT1 reside in Mitochondrial Associated Membrane of the ER (MAM) where phospholipid traffic between ER and mitochondria occurs (Figure 1.1). Contrary to MLCLAT1, ALCAT1 does reveal specificity for linoleic acid and incorporates long polyunsaturated acyl chains into cardiolipin. ALCAT1 action correlates with oxidative stress and damage because cardiolipin species formed by ALCAT1 are more susceptible to peroxidation ( $\mathrm{Ng}$ et al., 2005). Interestingly, observed consequences of ALCAT1 overexpression are oxidative stress, mitochondrial fragmentation and mtDNA instability (Li et al., 2012). Thus, ALCAT1 shows indications to link cardiolipin remodeling with oxidative stress and defects in mitochondrial fusion.

\subsubsection{Cardiolipin degradation}

The half-life of cardiolipin is relatively long, probably caused by the fact that cardiolipin interacts with protein complexes in mitochondrial membranes in cardiolipin-rich domains (Xu et al., 2014, 2016). Cardiolipin, which is not bound to protein complexes, is more accessible for degradation. The enzymes catalyzing the degradation of cardiolipin by deacylation are the members of the phospholipase family. One of the candidates is cytosolic $\mathrm{PLA}_{2}$, which degrades specifically monoand dilyso-cardiolipin but not cardiolipin (Buckland et al., 1998). The second candidate is phospholipase $\mathrm{D}$, which has been shown to hydrolyse cardiolipin in the outer mitochondrial membrane. However, the involvement of phospholipase D in cardiplipin turnover needs to be further clarified (Huang et al., 2011). The third 
candidate is phospholipase HSD10, which has been shown to detect oxidized cardiolipin and to mediate the cardiolipin degradation. Oxidized cardiolipin perturbes mitochondrial functions, indicating that HSD10 plays an important role of mitochondrial membrane homeostasis and functions (Boynton et al., 2015).

\subsection{Role of cardiolipin in mitochondrial functions}

\subsubsection{Mitochondria-targeted protein translocation via TOM and TIM23 complex.}

Most of the 1500 mitochondrial proteins are nuclear encoded and directed to the mitochondrial outer membrane by specific $\mathrm{N}$-terminal presequence and transported to their final destination in mitochondrial compartments (Chacinska et al., 2009; Morgenstern et al., 2017; Neupert et al., 2007; Voegtle et al., 2017). N-terminal presequences vary in size but have usually a length of 15-55 amino acids. The special property of $\mathrm{N}$-terminal presequence is a positively charged amphipathic $\alpha$-helix (von Heijne, 1986; Roise et al., 1986). The positive charge enables the translocation through inner mitochondrial membrane, which is electrostatically propelled by the membrane potential. The membrane potential is the driving force of mitochondrial protein import (Martin et al., 1991; Shariff et al., 2004; van der Laan et al., 2007).

The first translocase, which mediates recruiting of presequence-containing proteins is the translocase of the outer membrane (TOM). The TOM subunits, TOM20 and TOM22 act as receptors and recognize the hydrophobic and polar faces of the presequence (Saitoh et al., 2007; Yamano et al., 2008). From this point, proteins can be conducted to their final destination in the IMM, OMM, IMS or matrix. The structural integrity of the TOM complex and substrate translocation depends on remodeled cardiolipin (Figure 1.2).

Proteins, which are destined to the IMM or matrix are transported via the presequence pathway executed by the TIM23 complex (Figure 1.2). The precursor protein arises from the TOM $22^{\mathrm{IMS}}$ domain and interacts with TIM50 ${ }^{\mathrm{IMS}}$ with high affinity and can be conveyed to the TIM23 channel (Geissler et al., 2002; Schulz et al., 2011; Yamamoto et al., 2002). The TIM23 complex is embedded in the inner membrane and interacts with the translocase associated motor complex (PAM), facing the matrix side (Chacinska et al., 2009; Morgenstern et al., 2017; Neupert et al., 2007; Voegtle et al., 
2017). PAM drives the ATP-dependent precursor protein import and its association to TIM23 depends on cardiolipin (Tamura et al., 2006; van der Laan et al., 2007). The integrity of the TIM23 complex and the interaction with its receptor constituent TIM50 requires cardiolipin (Malhotra et al., 2017; Tamura et al., 2006; van der Laan et al., 2007). TIM23 and TIM17 form the channel but just TIM23 interacts with the substrate (Martinez-Caballero et al., 2007). Upon the protein import is ensured, Nterminal presequence-containing precursor proteins are processed via the mitochondrial processing peptidase (MPP) (von Heijne et al., 1989).

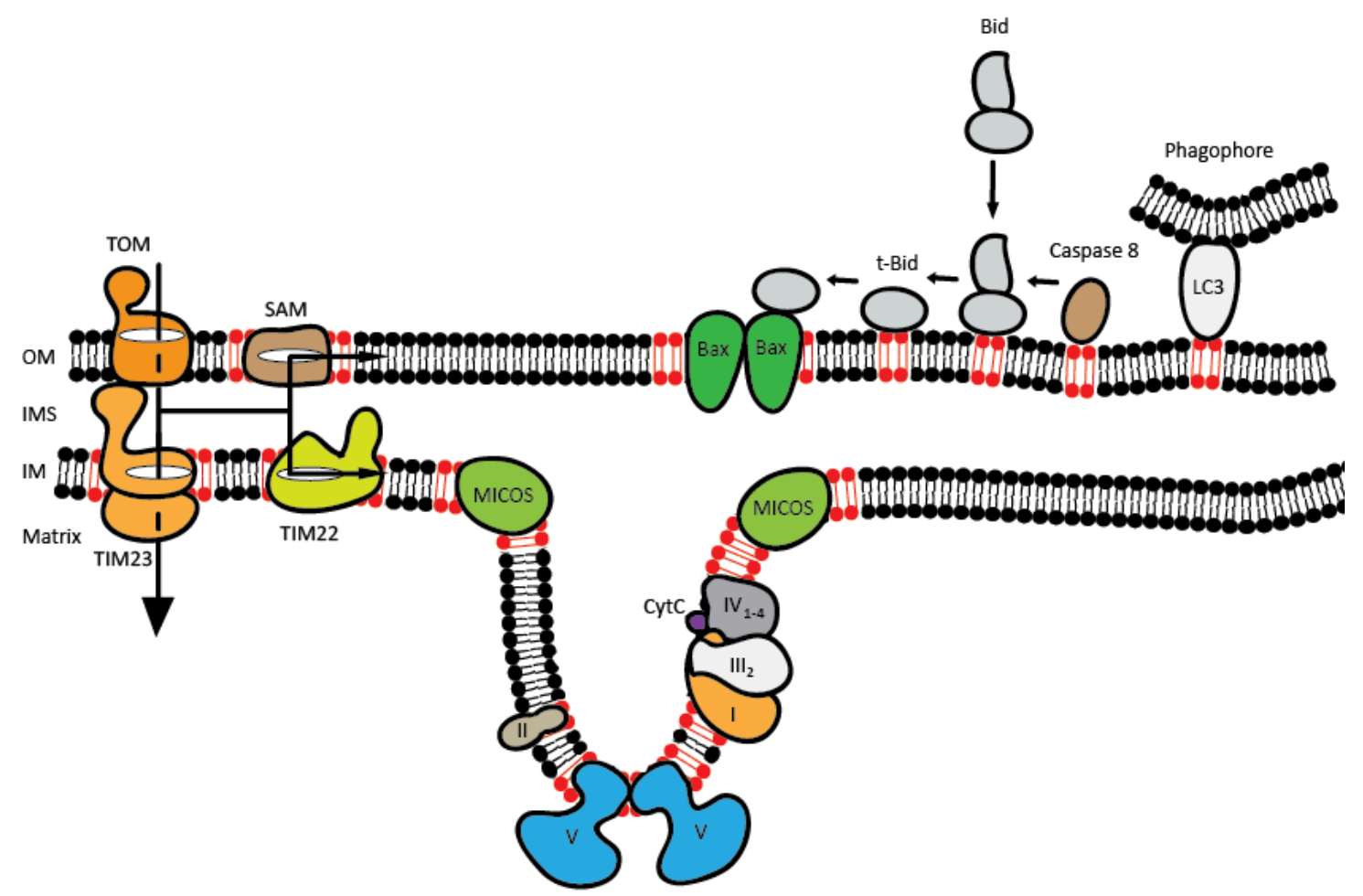

Figure 1.2: Cardiolipin participates and facilitates the key mitochondrial functions (Dudek et al., 2018).

Cardiolipin (red) plays a critical role in maintaining the structural integrity of the respiratory chain complexes, protein translocases and MICOS complex. Externalized cardiolipin domains act as a platform to initiate mitophagy by recruiting LC3 and apoptosis activation. IM: inner membrane, OM: outer membrane, IMS: intermembrane space

\subsubsection{Cardiolipin and its involvement in oxidative phosphorylation}

The key role of mitochondria is the conversion of chemical energy stored in the form of monosaccharides, lipids and amino acids into adenosine triphosphate (ATP) by 
oxidative phosphorylation. Around $95 \%$ of whole body ATP is generated by mitochondrial respiration (Dudek et al., 2018). The respiratory chain contains four protein complexes (CI-IV) and resides in the inner membrane. Reducing equivalents, such as NADH and $\mathrm{FADH}_{2}$, generated by catabolic pathways via oxidation of diverse carbon sources, transfer electrons on CI and CII, respectively. Those electrons are transported from CI and CII to CIII via ubiquinone. The soluble cytochrome c transfers electrons from CIII to the CIV for terminal reduction of molecular oxygen. The sequential redox reactions of the electron transport chain are exergonic reactions, which are coupled with endergonic pumping of protons from the matrix side to the intermembrane space via CI, CIII and CIV. The generated electromotive force or membrane potential drives the proton import back to the matrix through $\mathrm{CV}$ accompanied with ATP production. Cardiolipin was shown to be inevitable for appropriate activity of respiratory chain complexes (Fiedorczuk et al., 2016; Sharpley et al., 2006; Wenz et al., 2009). For instance, one molecule of cardiolipin binds to the interaction site of the CIII dimer and is tightly bound even after isolation of CIII (Palsdottir et al., 2004). Cardiolipin is also discussed of being involved in proton pumping of CIII and CIV. The negative charged "head" of cardiolipin could act as a trap for protons and resides close to the ubiquinone reduction site in CIII (Wenz et al., 2009). Moreover, cardiolipin has one binding site at the discussed proton-conducting channel in CIV and could be involved in proton pumping by CIV (Sedlak et al., 1999, 2006). Cardiolipin maintains the structural integrity of CIV and the CIII dimer, as well as the stability of CV oligomers. Cardiolipin binding is required for appropriate CV activity and may play a role in proton conduction through CV (Eble et al., 1990; Laird et al., 1986). CI and CII also require cardiolipin for their activity and structural integrity (Fiedorczuk et al., 2016; Schwall et al., 2012; Sharpley et al., 2006). CII levels are shown to be drastically reduced in tafazzin-deficient mouse hearts (Dudek et al., 2016).

CI, CIII and CIV form respiratory chain supercomplexes, which are also referred as respirasomes (Lenaz et al., 2009; Milenkovic et al., 2017; Schägger et al., 2002). Respirasomes increase the efficiency of the electron transport between redox pairs and may decrease the risk of reactive oxygen species (ROS) generation (Dudek et al., 2016). The lack of remodeled cardiolipin in different tafazzin-deficient models causes dissociation of respirasomes (Figure 1.2) (Brandner et al., 2005; Dudek et al., 2013; Zhang et al., 2002). 


\subsubsection{Role of cardiolipin: Mitophagy, apoptosis and morphology maintenance.}

In addition to the role on OXPHOS complexes, cardiolipin also affects other mitochondrial functions, such as apoptosis, function of metabolite carriers, mitochondrial morphology and mitophagy. Starting with mitophagy, mitophagy is a specific form of autophagy, which degrades damaged mitochondria by phagophore formation (Youle et al., 2012). Recruitment and activation of adapter proteins of the autophagic machinery to the outer membrane is facilitated by externalized cardiolipin under stress conditions. An increased content of cardiolipin in the outer membrane provides a signaling platform for LC3-dependent autophagosomal membrane recruitment (Figure 1.2) (Anton et al., 2016; Chu et al., 2013).

The cardiolipin externalization also plays a role in activation of apoptotic signaling at the outer membrane. Caspase- 8 can be recruited to the outer membrane, as long as cardiolipin is present. The pro-apoptotic member of the Bcl-2 family, Bid interacts with cardiolipin of the outer membrane and undergoes caspase-8-dependent processing (Figure 2.1). Thus, cardiolipin sustains the homeostasis of apoptotic Bcl-2 proteins at the outer mitochondrial membrane (Lutter et al., 2000).

The Mitochondrial contact site and cristae organizing system (MICOS) complex is located in the inner membrane and is critical for formation of cristae junctions and interaction with the outer membrane (van der Laan et al., 2012). Cardiolipin microdomains have been suggested to interact with MICOS components (Figure 1.2) (Koob et al, 2015; Weber et al., 2013).

Alterations in mitochondrial morphology caused by impairments in cardiolipin remodeling may lead to pleiotropic and severe physiological phenotypes. The consequence of impaired tafazzin function is illustrated by the Barth syndrome and is introduced in the following chapter.

\subsection{Barth syndrome (BTHS) - Pathophysiology of tafazzin deficiency and therapeutic approaches}

Barth syndrome (BTHS) is a monogenic and X-linked recessive syndrome, which shows an increased degree of infant mortality caused by heart failure or infections. 
The most characteristic symptoms are skeletal myopathy, dilated cardiomyopathy (DCM), neutropenia, growth retardation and high levels of 3-methylglutaconic acid in urine (Barth et al., 1983; Barth et al., 1999; Clarke et al., 2013; Spencer et al., 2006). The genetic origin of BTHS are pathogenic mutations including nonsense, missense, frameshift and splicing defects of the cardiolipin remodeling enzyme, TAZ (Bione et al., 1996; Ferri et al., 2016; Ronvelia et al., 2012). The X-chromosome-linked inheritance reasoned that most of the infant BTHS patients are male individuals, but even a female patient was reported (Cosson et al., 2012). The major clinical feature of BTHS is a left ventricular non-compaction (LVNC) associated with dilated cardiomyopathy (DCM) (Bunse et al., 2003; Merante et al., 1994; Sproudle and Kaufmann et al., 2008). Various additional abnormalities as sudden cardiac death, ventricular arrhythmia, lethargy, exercise intolerance, hypoglycemia may correlate with BTHS disease process (Spencer et al., 2005; 2006).

BTHS is characterized by reduced mature cardiolipin levels and an enhancement its precursor MLCL (Schlame et al., 2003). The MLCL/CL ratio serves to diagnose BTHS in patients and BTHS models (Houtkooper et al., 2009). On molecular level, different BTHS models show remodeling of respiratory chain complexes. These molecular phenotype correlates with decreased mitochondrial membrane potential and a reduction in respiratory performance (Dudek et al., 2016; Gonzalvez et al., 2013; McKenzie et al., 2006). Deficiency of cardiolipin remodeling causes alterations in mitochondrial morphology and altered cristae structure formation (Acehan et al., 2011). BTHS patient skeletal muscle, heart and liver biopsies show malformed mitochondria and fragmented and circular cristae bundles (Barth et al., 1983; Bissler et al., 2002; Orstavik et al., 1998).

Various therapeutic strategies are developed on the basis of these mitochondrial features. The aim of all therapeutic approaches is to enrich the pool of cardiolipin in relation to its precursor MLCL. First pharmacological inhibition of phospholipase activity in BTHS mouse models could rescue reduced cardiolipin levels (Malhotra et al., 2009). Second, administration of the precursor linoleic acid rescued sarcomere structure in BTHS iPSC-derived cardiomyocytes (Wang et al., 2014) and increased cardiolipin levels in BTHS fibroblasts (Valianpour et al., 2002). The overexpression of the cardiolipin-synthesizing enzyme MLCLAT1 in BTHS lymphoblasts could reduce reactive oxygen (ROS) production and increase cardiolipin level but did not revert the respiratory chain remodeling (Mejia et al., 2018). ROS scavenging is an 
additional mechanism to counteract the molecular phenotypes in BTHS. Szeto et al. could rescue mitochondrial respiration and cristae morphology (Szeto et al., 2014). The Szeto-Schiller peptide (SS-31) interacts with cardiolipin directly, accumulates at mitochondrial membranes and scanvenges ROS. Moreover, Wang et al. also used MitoTEMPO treatment for ROS scavenging leading to improved sarcomere organisation in patient iPSC-derived cardiomyocytes (Wang et al., 2014).

Besides the above addressed roles of cardiolipin, the mitochondrial calcium homeostasis is known to depend on sufficient amounts of remodeled cardiolipin. In fact, Kamer et al. demonstrated that the Mitochondrial Calcium Uniporter Uptake 1/2 dimer (MICU1/2), a Mitochondrial Calcium Uniporter (MCU) component, requires cardiolipin to be associated with the inner mitochondrial membrane (Kamer et al., 2017). The following chapter highlights the importance of MCU complex stability and constitution in connection to mitochondrial calcium metabolism.

\subsection{The role of mitochondria in calcium metabolism}

Mitochondrial calcium uptake and release mechanisms play a crucial role in cellular calcium homeostasis. Intracellular calcium can be uptaken and stored in the mitochondrial matrix preventing excess calcium-stimulated metabolism. Calcium is a second messenger of intracellular signal transduction and regulation (Belosludtsev et al., 2019; Giorgi et al., 2018; Kwong et al., 2017). Mitochondria are considered as one of the organelles, which regulates cellular calcium levels. Mitochondrial activity and metabolism are stimulated by calcium. Beside its participation in bioenergetics, calcium also plays a role in ROS production (Bertero et al., 2018), cell cycle regulation and proliferation (Koval et al., 2019), cell contractility (Eshima et al., 2018), migration (Paupe et al., 2018; Zheng et al., 2017), and the mitochondrial permeability transition pore (mPTP) opening (Penna et al., 2018).

Calcium homeostasis plays a critical regulatory role in excitation-contraction coupling in cardiac and skeletal muscle cells. The calcium-dependent excitation of the muscle or cardiac cell facilitates contraction, based on rhythmic cycles of calcium release/uptake to the cytosol and subsequent buffering by uptake into organelles, such as sarcoplasmic reticulum (SR) and mitochondria. The clearance of cytosolic calcium 
into the SR after an excitation-contraction cycle is performed by the sarcoplasmic/endoplasmic reticulum calcium ATPase (SERCA) in cardiac and skeletal muscle cells. Surprisingly, the calcium uptake by mitochondria is lower than $1 \%$ in cardiomyocytes. Conversely, in non-muscle cells, the Mitochondrial Calcium Uniporter undertakes a more weighted role in calcium clearance and performs intracellular calcium buffering by calcium uptake into the mitochondrial matrix (Bers et al., 2002; Fieni et al., 2012).

\subsection{The Mitochondrial Calcium Uniporter (MCU) complex}

\subsubsection{Overview and function}

Excess cellular calcium can be uptaken into the mitochondrial matrix by the Mitochondrial Calcium Uniporter, which is forced by the electrochemical gradient (Baughman et al., 2011; De Stefani et al., 2011). Hence, mitochondria are able to buffer excess cellular calcium and stimulate energy catabolism depending on metabolic demands. The MCU complex is a calcium-sensing and -transducing channel spanning the inner mitochondrial membrane (Figure 1.3) (Baughman et al., 2011; Kirichok et al., 2004; Tsai et al., 2016; Wang et al., 2019).

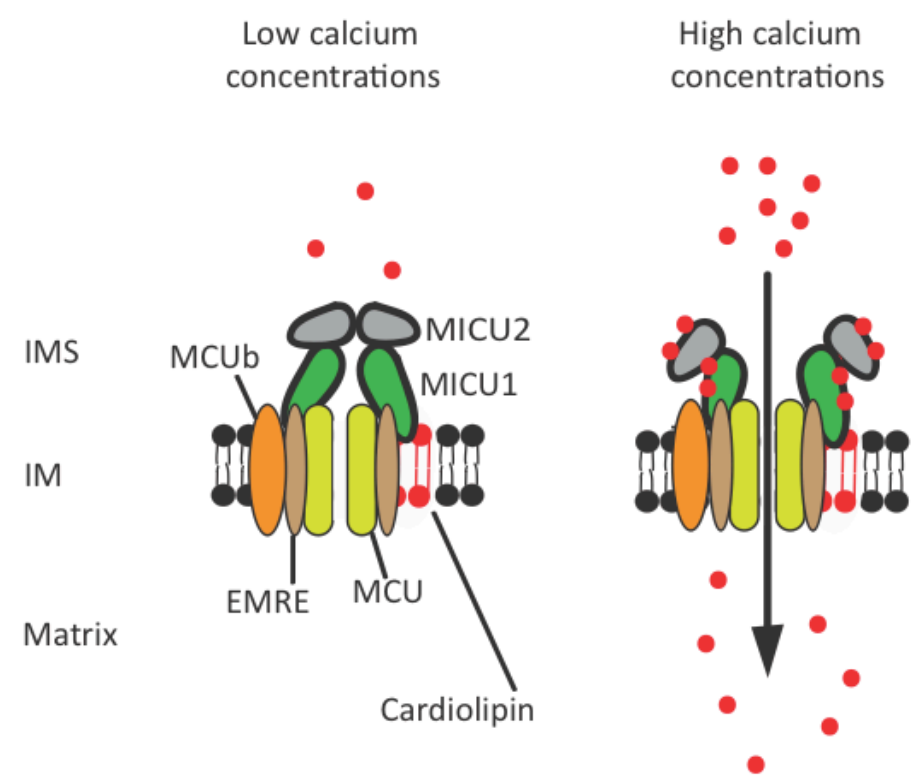

Figure 1.3: MCU components and their concerted actions to uptake cellular calcium. 
The main constituents of the Mitochondrial Calcium Uniporter complex are the integral membrane proteins $\mathrm{MCU}, \mathrm{EMRE}$ and $\mathrm{MCUb}$ forming the calcium-conducting pore in the IMM and the IMS-located MCU gatekeepers MICU1 and MICU2. Increased calcium transients cause dissociation of the gatekeepers from the pore and enable calcium conductance into mitochondrial matrix. MICU1/2 dimer is discussed to be IMM-associated via cardiolipin (Kamer et al., 2017).

The uniporter complex consists of six main constituents which are well studied, both functionally and structurally (Baradaran et al., 2018; Fan et al., 2018; Yoo et al., 2018). The core subunit of MCU is the MCU protein. It forms homooligomers, which generate the pore-forming subunit within the inner membrane. Cellular calcium is sensed in the IMS by calcium-binding EF-hand domains of inner membraneassociated gatekeeper proteins Mitochondrial Calcium Uptake 1 and 2 (MICU1 and MICU2) (Kamer et al., 2017; Liu et al., 2016; Paillard et al., 2018). Calcium binding to MICU1/2 exerts conformational changes, which are mediated to the Calciumconducting MCU pore through the integral inner membrane protein, Essential MCU regulator (EMRE) (Tsai et al., 2016). Two further transmembrane proteins interacting with MCU are MCUb and MCUR1, have been shown to exert tissue-specific regulatory mechanisms of mitochondrial calcium uptake (Oxenoid et al., 2016; Tsai et al., 2016; Wang et al., 2019).

In the next sections, these six MCU components, their interplay and the orchestrating actions in mitochondrial calcium uptake are introduced in more detail.

\subsubsection{Mitochondrial Calcium Uniporter (MCU) protein}

The Mitochondrial Calcium Uniporter protein (MCU) consists of two transmembrane spans permeating the inner mitochondrial membrane and is composed of 350 amino acids $(39,7 \mathrm{kDa})$. Structural informations shed light into the architecture of the uniporter domains. The structure of the N-terminal domain of MCU (MCU-NTD) was determined using nuclear magnetic resonance (NMR), while the coiled coil domain (CCD), the transmembrane domain (TMD) and the MCU-NTD were solved by cryoelectron microscopy (Cryo-EM). Four MCU protein monomers form a tetrameric ionselective pore and topology-wise, $\mathrm{N}$ - and $\mathrm{C}$-terminus of $\mathrm{MCU}$ face the matrix site (Baradaran et al., 2018; Fan et al., 2018; Oxenoid et al., 2016; Yoo et al., 2018). The 
TMD contains two transmembrane spans (TM1 and TM2). TM1 interacts directly with EMRE and TM2 forms the calcium pore (Fan et al., 2018). The two TM spans of MCU are linked by a loop in the intermembrane space and contain the highly conserved DXXE motive (X are hydrophobic amino acids). Thereby, aspartate (D) and glutamate $(\mathrm{E})$ residues of each $\mathrm{MCU}$ monomer form acidic rings and attract calcium selectively to the pore (Baughman et al., 2011; de Stefani et al., 2011; Oxenoid et al., 2016; Phillips et al., 2019).

\subsubsection{Mitochondrial Calcium Uptake 1 protein (MICU1)}

Mitochondrial Calcium Uptake 1 protein (MICU1) is a regulatory protein of MCU. Three MICU1 isoforms exist in mouse tissues composed of around 477 amino acids, having a molecular weight of around $55 \mathrm{kDa}$. MICU1 expression is ubiquitous, whereas the splicing isoform MICU1.1, containing an additional micro-exon between exons 5 and 6, is just expressed in brain and skeletal muscle (Vecellio Reane et al., 2016). MICU1 operates as a molecular gatekeeper of MCU while interacting with the DIME sequence of MCU at low calcium currents. This results in an inhibition of calcium uptake through the inner mitochondrial membrane (Kamer et al., 2017; Liu et al., 2016; Paillard et al., 2018). At higher cellular calcium levels, the MICU1 binding site towards MCU can be blocked by chelated calcium. MICU1 is a typical calciumbinding protein and contains two EF-hand domains. These domains are helix-loophelix motifs and are separated by an alpha helix (Kamer et al., 2017). This structural constitution enables divalent cation-chelating specificity. The cation-ligand interaction of both MICU EF-hands is specific to calcium, but not to manganese (Kamer et al., 2018). Kamer et al. revealed in 2018, that manganese can pass the MCU pore in a MICU1-KO situation (HEK293T cells), but is incapable to activate the conformational change of MICU1 and mediation of MCU pore opening (Kamer et al., 2018). The calcium-binding specificity of MICU1 was also shown in the yeast model system $S$. cerevisiae where coexpression of MICU1 and MCU prevents manganese forwarding through the inner mitchondrial membrane. Thus, the divalent cation-specificity of MICU1 prohibits mitochondrial manganese overload and toxicity (Kamer et al., 2018; Wettmarshausen et al., 2018). Lacking MICU1 causes mitochondrial calcium overload and can induce myopathic and neurological disorders in mice (Liu et al., 2016). In-vivo MICU1 deficiency leads to elevated resting 
mitochondrial calcium levels, decreased ATP production and changes in mitochondrial morphology. Similar phenotypes could be observed in MICU1deficient patients (Liu et al., 2016). MICU1 does not act alone to mediate calciumbinding. It is capable to form homodimers and heterodimers with its paralog MICU2 via disulfide bonds at the C-terminal domains, respectively (Kamer et al., 2017; Petrungaro et al., 2015). The disulfide bond is introduced by the oxidoreductase Mia40 in the IMS and requires MCU as a platform for MICU dimerization. At low calcium levels, MICU-dimers can interact with the pore-forming subunit of MCU. Conversely, upon increased calcium concentrations the MICU-dimer dissociates from the MCU pore. This finding implicates that MCU complex formation and stability take place in a calcium-dependent manner (Petrungaro et al., 2015). The membrane association of MICU1/2-dimer could spring from its selective interaction to the phospholipid cardiolipin, which was shown with cardiolipin-containing liposomes (Figure 1.3) (Kamer et al., 2017). Both, MICU1 and MICU2 have high affinity to calcium and more aspects of the cooperative action of uniporter-dependent calcium signal transduction in mitochondria are approached in the following chapter.

\subsubsection{Mitochondrial calcium uniporter regulator 1 (MCUR1)}

Mitochondrial calcium uniporter regulator 1 (MCUR1) occurs in three different isoforms in mouse tissues (38 kDa, $31 \mathrm{kDa}$ and 18,6 kDa;). MCUR1 also consists of two transmembrane domains similar to MCU, but N- and C-terminus face the IMS (Adlakha et al., 2019; Tomar et al., 2016). It is debatable whether it forms a core subunit of MCU. Originally, MCUR1 was published by Mallilankaraman et al. in 2012 as a component of MCU, inevitable for mitochondrial calcium uptake and stimulation of mitochondrial bioenergetics (Mallilankaraman et al., 2012). Later on, MCUR1 was published by E. Shoubridge lab of not being a MCU component and direct regulator, but more of an assembly factor of cytochrome c oxidase assembly (CIV) (Paupe et al., 2015). Suppression of MCUR1 in human fibroblasts causes depleted mitochondrial membrane potential, a CIV assembly defect and decreased mitochondrial calcium uptake (Paupe et al., 2015). Subsequently, it was shown that MCUR1 was an interaction partner of the MCU protein and an essential scaffold factor. MCUR1 acted as a positive regulator for MCU complex assembly, mitochondrial calcium import and stimulation of calcium-dependent mitochondrial 
metabolism (Adlakha et al., 2019; Chaudhuri et al., 2016; Mallilankaraman et al., 2015; Tomar et al., 2016; Vais et al., 2015). Structurally, MCUR1 contains two transmembrane spans and binds directly to the MCU-NTD (Adlakha et al., 2019). Depletion of MCUR1 via organ-specific knockout causes impaired calcium uptake capacity, reduced ATP production and increased autophagy in cardiomyocytes and endothelium cells (Tomar et al., 2016).

\subsubsection{Mitochondrial Calcium Uniporter subunit b (MCUb)}

Mitochondrial Calcium Uniporter subunit b (MCUb) is a dominant-negative constituent of MCU, which acts as a regulator of the Calcium channel activity of MCU (Checchetto et al., 2019; Lambert et al., 2019; Raffaello et al., 2013). Murine MCUb is composed of 345 amino $(39,8 \mathrm{kDa})$ and similar to MCU, MCUb is a putative two-transmembrane-spanned and integral membrane protein. MCUb forms a heterooligomeric pore with MCU but does not interact with the gatekeepers MICU1 and MICU2 (Checchetto et al., 2019; Lambert et al., 2019). As a consequence, MCUb alone is not capable to organize a calcium-sensing and -conductive channel through the inner mitochondrial membrane (Raffaello et al., 2013). MCUb plays an important role for mitochondrial calcium homeostasis because MCU-to-MCUb stoichiometry modulates mitochondrial calcium uptake. For instance, overexpression of MCUb in adult cardiac myocytes displaces MCU and its interacting gatekeepers MICU1 and MICU2 from the inner membrane, with the consequence that the MCU complex size is lowered. Hence, cooperative gatekeeper-dependent activation of MCU is diminished and the calcium-conductive function is reduced (Lambert et al., 2019). Paillard et al. showed that the MCU-to-MCUb ratio varies among different tissues and metabolic demands. For instance, the MCU-to-MCUb ratio is relatively high in skeletal muscle and reduced in cardiac tissues (Paillard et al., 2017). Thus, MCU-toMCUb stoichiometry determines the molecular composition and function of the uniporter complex and could be a regulatory mechanism to adapt to different physiologic conditions.

\subsubsection{EMRE, the Essential MCU Regulator}

The Essential Mitochondrial Calcium Uniporter Regulator (EMRE or SMDT1) is a 
TMD-containing inner mitochondrial protein composing of 107 amino acids (11 $\mathrm{kDa}$ ). Its $\mathrm{N}$-terminus faces the mitochondrial matrix and the $\mathrm{C}$-terminal domain is exposed to the IMS, respectively (Tsai et al., 2016; Wang et al., 2019; Yamamoto et al., 2016). Cryo-EM analysis of human MCU-EMRE complex structure demonstrates that one EMRE interacts with one MCU subunit. The resulting structure is a MCUEMRE tetramer, which turns out as the minimum composition for a calciumpermeable uniporter. Furthermore, EMRE is needed to initiate the oligomerizaton of two MCU-EMRE tetramers to a higher-order MCU complex. The dimerization of two MCU-EMRE complexes occurs along the MCU-N-terminal domains (NTDs) in the mitochondrial matrix. This MCU-EMRE oligomer is stabilized by the beta-hairpin structure of the N-terminal domain of EMRE. This EMRE-dependent oligomerization enables the coordination between proximal MCU complexes and functional coupling (Wang et al., 2019). Functional MCU in metazoans requires EMRE, whereas some primitive eukaryotes do not contain EMRE for appropriate MCU function (KovacsBogdan et al., 2014; Wang et al., 2019). In response to increased intracellular calcium transients, EMRE mediates the calcium-sensing function of MICU1 and MICU2 in the IMS with the calcium-conducting function of the MCU pore. This is enabled by the interaction of the conserved polyaspartic tail of EMRE (C-terminus) with MICU1 in the IMS (Tsai et al., 2016).

In conclusion, EMRE exerts a dual functionality. First, it orchestrates the uniporter response to cytosolic calcium signaling via its physical connection to MICU1 and second, it stabilizes and activates the MCU pore opening (Tsai et al., 2016).

\subsubsection{Proteolytic regulation of MCU composition and activity}

MCU assembly, function and turnover were shown to be regulated on transcriptional, post-transcriptional and post-translational levels (Nemani et al., 2018). Turnover of EMRE is facilitated by the mitochondrial m-AAA metalloproteases AFG3L2 and SPG7 (ATPases associated with diverse cellular activities) (König et al., 2016; Opalinska et al., 2018). Once assembled in MCU, EMRE degradation is inhibited. Loss of AFG3L2 causes inhibition of EMRE degradation and accumulation of constitutively active MCU-EMRE sub-complexes (Tsai et al., 2017). Excess MCUEMRE subcomplexes could cause calcium leakage into mitochondria, mitochondrial permeability transition pore (mPTP) opening and neuronal death in mouse brain 
(König et al., 2016). Moreover, SPG7 KO cells reveal elevated EMRE protein levels and alterations in the MCU stoichiometry (Hurst et al., 2019).

\subsection{State of art}

The aim of the following study has been the characterization of MCU in different

Barth Syndrome Models under biochemical perspectives. Recent studies about the structural integrity of mitochondrial protein complexes showed that respiratory chain complexes, translocases, metabolite carriers, MICOS complexes, etc. were depended on appropriate action of tafazzin (Dudek et al, 2016; Dudek et al., 2018). The dependency of MCU function, complex assembly and stability on tafazzin has never been investigated before in mammalian Barth Syndrome models. The importance of MCU in the role of mitochondrial and cellular calcium homeostasis in cardiac and skeletal muscle function is well studied. Therefore, it was worth checking the biochemical properties of MCU in tafazzin lacking models. Two mammalian Barth Syndrome models were used in this study. First, WT and TAZ KO MEF cells, a murine clonal cell line, and second, different organs from the Rosa shTAZ/BTHS mouse. The respiratory chain complexes are remodeled in BTHS cardiomyocytes. Steady state levels of complex II are specifically reduced in BTHS mouse hearts (Dudek et al., 2016). Putative alterations in the expression levels of MCU constituents or complex composition and organization of MCU could affect MCU function and mitochondrial calcium homeostasis. These open questions was approached by BlueNative PAGE analysis of MCU complex organization in tafazzin-depleted models and interaction studies of MCU constituents via coimmunprecipitation.

The impact of reactive oxygen species (ROS) on MCU activity and complex composition is still under debate (Dong et al., 2019). ROS levels are increased in TAZ KO MEF cells (Chowdhury et al., 2018) and BTHS cardiomyocytes (Dudek et al., 2016). Therefore, MCU complexes in TAZ KO MEF mitochondria upon ROS scavenging will be investigated.

The impact of remodeled cardiolipin on translocase stability and activity is not well studied. The import and assembly process of MCU components in TAZ KO MEF mitochondria will be addressed. 


\section{Materials and methods}

\subsection{Materials}

\subsubsection{Chemicals}

Table 2.1 Chemicals

\begin{tabular}{|l|l|}
\hline Chemicals & Supplier \\
\hline$\left[{ }^{35}\right.$ S]-L-methionine & $\begin{array}{l}\text { Hartmann Analytic (Braunschweig, } \\
\text { Germany) }\end{array}$ \\
\hline 2-Mercaptoethanol & Sigma-Aldrich \\
\hline 4- Nitro blue tetrazolium chloride & Roth \\
\hline 6-Aminocaproic acid & Sigma-Aldrich \\
\hline Acetic acid & Roth \\
\hline Acetone & Roth \\
\hline Acrylamide/bisacrylamide (37.5:1) solution & Roth \\
\hline Acrylamide, 2x crystallized & Roth \\
\hline Agarose NEEO ultra-quality & Roth \\
\hline Ampicillin & AppliChem \\
\hline Anti-FLAG M2 Affinity Gel & Sigma-Aldrich \\
\hline Antimycin A & Sigma-Aldrich \\
\hline ATP (Adenosine-5'-triphosphate) & Roche \\
\hline Bis-Tris Buffer grade & AppliChem \\
\hline Bovine serum albumin (BSA) & Sigma-Aldrich \\
\hline Bromophenol Blue & Merck \\
\hline Cardiolipin & Avanti \\
\hline Chloroform & Roth \\
\hline cOmplete, EDTA-free protease inhibitor & \\
\hline Tablet & Serva \\
\hline Coomassie Brilliant Blue R-250 & Roche \\
\hline
\end{tabular}




\begin{tabular}{|l|l|}
\hline Copper(II)sulfate pentahydrate & Merck \\
\hline Creatine kinase & Roche \\
\hline Creatine phosphate & Roche \\
\hline Coomassie Brilliant Blue R-250 & Serva \\
\hline Cycloheximide & Applichem \\
\hline Cytochrome $c$ & Calbiochem \\
\hline Diaminobenzidine & Sigma-Aldrich \\
\hline Digitonin & Calbiochem \\
\hline Dipotassium phosphate & Roth \\
\hline Disodium phosphate & Roth \\
\hline DMSO (dimethylsulfoxide) & Applichem \\
\hline EDTA & Roth \\
\hline EGTA & Roth \\
\hline Ethanol & Roth \\
\hline Ethidium Bromide (0.025\%) & Roth \\
\hline Fetal Bovine Serum & Biochrom \\
\hline Glucose & Roth \\
\hline Glutamine & Life Technologies \\
\hline Glycerol & Sigma-Aldrich \\
\hline Glycine & Roth \\
\hline HEPES & Roth \\
\hline Hydrochloric acid, 37\% & Roth \\
\hline Hydrogen peroxide & Sigma-Aldrich \\
\hline IgG from human serum & Sigma-Aldrich \\
\hline IgG protein standard & BioRad \\
\hline Imidazole & Roth \\
\hline Magnesium acetate & Merck \\
\hline Magnesium chloride & Rerck \\
\hline Magnesium sulfate & Applichem \\
\hline Malate & \\
\hline Mannitol & Rotha-Aldrich \\
\hline Methanol & \\
\hline
\end{tabular}




\begin{tabular}{|c|c|}
\hline Methionine & Roth \\
\hline MOPS & Roth \\
\hline NADH & Roche \\
\hline $\begin{array}{l}\text { N,N-Methylenebisacrylamide, } \\
\text { crystallized }\end{array}$ & Serva \\
\hline Oligomycin & Sigma \\
\hline o-phosphoric acid & Merck \\
\hline Opti-Mem & Life Technologies \\
\hline OrangeG & Sigma-Aldrich \\
\hline Oxaloacetic acid & Sigma-Aldrich \\
\hline Penicillin Streptomycin & Life Technologies \\
\hline Phenol & Roth \\
\hline Phenol-Chloroform & Roth \\
\hline Phosphatidylcholine & Avanti \\
\hline Phosphatidylethanolamine & Avanti \\
\hline Phosphatidylglycerol & Avanti \\
\hline PMSF & Roth \\
\hline Potassium chloride & Roth \\
\hline Potassium cyanide & Sigma-Aldrich \\
\hline Potassium hydrogen phosphate & Merck \\
\hline Potassium dihydrogen phosphate & Roth \\
\hline Protein-A sepharose & GE Healthcare \\
\hline Proteinase $\mathrm{K}$ & Roche \\
\hline PVDF membrane (Immobilon-P) & Merck \\
\hline Roti-Quant ${ }^{\circledR}$ reagent & Roth \\
\hline SDS (sodium dodecyl sulfate) & Serva \\
\hline SDS marker broad range & Biorad \\
\hline SDS-PAGE protein standard & Serva \\
\hline Sodium chloride & Roth \\
\hline Sodium hydroxide & Roth \\
\hline Sodium pyruvate & Sigma-Aldrich \\
\hline Sodium succinate & Sigma-Aldrich \\
\hline
\end{tabular}




\begin{tabular}{|l|l|}
\hline Sucrose & Roth \\
\hline Tetramethylethylenediamine (TEMED) & Roth \\
\hline Tetracycline hydrochloride & Sigma-Aldrich \\
\hline Trehalose & Roth \\
\hline Tricine & Roth \\
\hline Trichloroacetic acid & Roth \\
\hline Triethylamine & Roth \\
\hline Tris (tris(hydroxymethyl)aminomethane) & Roth \\
\hline Triton-X-100 & Roth \\
\hline Tween-20 & Roth \\
\hline Urea & Roth \\
\hline Valinomycin & Sigma-Aldrich \\
\hline
\end{tabular}

\subsubsection{Buffers and solutions}

Table 2.2 Buffers and solutions

\begin{tabular}{|c|c|}
\hline${ }^{3}$ S-labeling medium & $\begin{array}{l}\text { DMEM (Dulbecco's Modified Eagle Medium) } \\
\text { with or without fetal calf serum, without } \\
\text { sodium pyruvate, L-Glutamine, L-Methionine } \\
\text { and L-Cysteine (filtered) }\end{array}$ \\
\hline AVO mix & $\begin{array}{l}0.8 \mathrm{mM} \text { antimycin, } 0.1 \mathrm{mM} \text { valinomycin, } 2 \mathrm{mM} \\
\text { oligomycin in ethanol }\end{array}$ \\
\hline Blotting buffer & $\begin{array}{l}\text { 20mM Tris, } 150 \mathrm{mM} \text { glycine, } 0.02 \% \text { SDS, } 20 \% \\
\text { methanol }\end{array}$ \\
\hline BN anode buffer & 50mM Bis-Tris/HCl pH 7.0 \\
\hline BN cathode buffer & $\begin{array}{l}50 \mathrm{mM} \text { Tricine, } \mathrm{pH} 7.0,15 \mathrm{mM} \text { Bis-Tris, with or } \\
\text { without } 0.02 \% \text { Coomassie Brilliant Blue G- } 250\end{array}$ \\
\hline BN gel buffer & $\begin{array}{l}\text { 66.67mM } 6 \text {-aminocaproic acid, } 50 \mathrm{mM} \text { Bis- } \\
\text { Tris/HCl pH } 7.0\end{array}$ \\
\hline
\end{tabular}




\begin{tabular}{|c|c|}
\hline BN sample loading buffer & $\begin{array}{l}\text { 0.5\% Coomassie Brilliant Blue G-250, 50mM 6- } \\
\text { aminocaproic acid, 10mM Bis-Tris, pH 7.0 }\end{array}$ \\
\hline BN solubilization buffer & $\begin{array}{l}\text { 1\% Digitonin, } 20 \mathrm{mM} \text { Tris } / \mathrm{HCl} \mathrm{pH} 7.4,0.1 \mathrm{mM} \\
\text { EDTA, pH 8, 50mM NaCl, } 10 \% \text { Glycerol, } 1 \mathrm{mM} \\
\text { PMSF }\end{array}$ \\
\hline Cell culture medium & $\begin{array}{l}\text { DMEM (Dulbecco's Modified Eagle Medium) } \\
\text { supplemented with } 10 \%(\mathrm{v} / \mathrm{v}) \text { fetal calf serum } \\
\text { (FCS), } 1 \mathrm{mM} \text { sodium pyruvate, } 2 \mathrm{mM} \text { L- } \\
\text { Glutamine, and with or without penicillin } \\
\text { streptomycin (filtered) }\end{array}$ \\
\hline Coomassie staining solution & $\begin{array}{l}2.5 \mathrm{~g} / \mathrm{L} \text { Coomassie Brilliant } \text { Blue } \mathrm{R}-250,40 \% \\
\text { ethanol, } 10 \% \text { acetic acid }\end{array}$ \\
\hline Coomassie destaining solution & $40 \%$ ethanol, $10 \%$ acetic acid \\
\hline Hypertonic buffer & 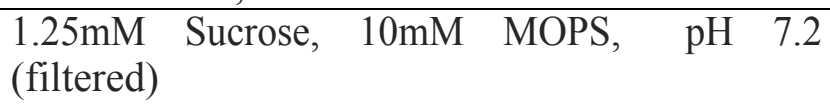 \\
\hline Hypotonic buffer & 100mM Sucrose, 10mM MOPS, pH 7.2, 1mM \\
\hline Import buffer & $\begin{array}{l}250 \mathrm{mM} \text { Sucrose, } 5 \mathrm{mM} \text { magnesium acetate, } \\
80 \mathrm{mM} \text { potassium acetate, } 5 \mathrm{mM} \text { methionine, } \\
10 \mathrm{mM} \text { sodium succinate, } 5 \mathrm{mM} \text { ATP, } 20 \mathrm{mM} \\
\text { Hepes, pH } 7.4\end{array}$ \\
\hline $\begin{array}{l}\text { Isolation buffer for mitochondria } \\
\text { isolation from mouse tissues }\end{array}$ & $\begin{array}{l}20 \mathrm{mM} \text { Hepes } \mathrm{pH} 7,6(0,95 \mathrm{~g} / 200 \mathrm{~mL}) \\
220 \mathrm{mM} \text { Mannitol 88,02g/200 mL) } \\
1 \mathrm{mM} \text { EDTA }(74 \mathrm{mg} / 200 \mathrm{~mL}) \\
70 \mathrm{mM} \text { Mannitol }(4,79 \mathrm{~g} / 200 \mathrm{~mL}) \\
\text { In } 50 \mathrm{~mL} \text { add } 1 \mathrm{~mL} \text { Roche protease inhibitor }\end{array}$ \\
\hline Isolation buffer & $\begin{array}{l}75 \mathrm{mM} \text { Mannitol, } 225 \mathrm{mM} \text { Sucrose, } 10 \mathrm{mM} \\
\text { MOPS, pH 7.2, 1mM EGTA (filtered) }\end{array}$ \\
\hline Krebs-Ringer solution & $\begin{array}{l}135 \mathrm{mM} \mathrm{NaCl}, 5 \mathrm{mM} \mathrm{KCl}, \mathrm{MgSO}_{4} 1 \mathrm{mM} \text {, } \\
\mathrm{K}_{2} \mathrm{HPO}_{4} \text { 0,4 mM, Glucose 5,5 mM, HEPES } 20 \\
\mathrm{mM}, \mathrm{pH} 7,4,1 \mathrm{mM} \mathrm{CaCl}_{2}\end{array}$ \\
\hline Lysogeny broth (LB medium) & $1 \% \mathrm{NaCl}, 0,5 \%$ yeast extract, $1 \%$ tryptone \\
\hline Potassium phosphate buffer, $\mathrm{pH} 7.4$ & $19 \% \mathrm{KH}_{2} \mathrm{PO}_{4}, 81 \% \mathrm{~K}_{2} \mathrm{HPO}_{4}$ \\
\hline
\end{tabular}




\begin{tabular}{|c|c|}
\hline PBS (phosphate-buffered saline) & \\
\hline Resolving gel (BN-PAGE) & $\begin{array}{l}137 \mathrm{mM} \mathrm{NaCl}, 2.7 \mathrm{mM} \mathrm{KCl}, 10 \mathrm{mM} \mathrm{Na} \mathrm{HPO}_{4} \text {, } \\
1.8 \mathrm{mM} \mathrm{KH}_{2} \mathrm{PO}_{4} \text { with or without } 1 \mathrm{mM} \mathrm{EDTA} \\
\text { (autoclaved) }\end{array}$ \\
\hline Resolving gel (SDS-PAGE) & $\begin{array}{l}\text { 4-16.5\% acrylamide/bisacrylamide (49.5\%/3\%), } \\
1 \times \text { BN gel buffer (see above), 2-30\% Glycerol }\end{array}$ \\
\hline Resolving gel (Tricine-SDS-PAGE) & $\begin{array}{l}\text { 10-18\% acrylamide/bisacrylamide (49.5\%/3\%), } \\
1 x \text { Tris-Tricine gel buffer (see below), } 0-13 \% \\
\text { glycerol }\end{array}$ \\
\hline ROS assay buffer & $\begin{array}{l}20 \mathrm{mM} \text { Tris, } 150 \mathrm{mM} \mathrm{NaCl}, 0.1 \% \text { Triton, } 3 \mathrm{mM} \\
\text { ADP, } 10 \mathrm{mM} \text { Succinate, } \mathrm{pH} 7.4\end{array}$ \\
\hline Stacking gel (BN-PAGE) & $\begin{array}{l}2 \% \text { acrylamide/bisacrylamide }(49.5 \% / 3 \%), 1 \mathrm{x} \\
\text { BN gel buffer }\end{array}$ \\
\hline Stacking gel (SDS-PAGE) & $\begin{array}{l}5 \% \text { acrylamide/bisacrylamide }(37.5 / 1), 80 \mathrm{mM} \\
\text { Tris- } \mathrm{HCl} \mathrm{pH} 6.8,0.1 \% \text { SDS }\end{array}$ \\
\hline Stacking gel (Tricine-SDS-PAGE) & $\begin{array}{l}4 \% \text { acrylamide/bisacrylamide }(49.5 \% / 3 \%), 1 \mathrm{x} \\
\text { Tris-Tricine gel buffer }\end{array}$ \\
\hline SDS running buffer & $25 \mathrm{mM}$ Tris, $192 \mathrm{mM}$ glycine, $0.1 \% \mathrm{SDS}$ \\
\hline SDS sample buffer & $\begin{array}{l}10 \% \text { glycerol, } 2 \% \text { SDS, } 0.01 \% \text { bromophenol } \\
\text { blue, } 60 \mathrm{mM} \text { Tris/HCl pH } 6.8,1 \% \text { beta- } \\
\text { mercaptoethanol }\end{array}$ \\
\hline SEM buffer & $\begin{array}{l}\text { 250mM sucrose, 1mM EDTA, 10mM EDTA, } \\
\text { pH } 7.2\end{array}$ \\
\hline TAE buffer & 40mM Tris/acetate $\mathrm{pH} 8.0,2 \mathrm{mM}$ EDTA \\
\hline TBS (Tris-buffered saline) & 20mM Tris/HCl, $\mathrm{pH} 7.5,125 \mathrm{mM} \mathrm{NaCl}$ \\
\hline TBS-T & $\begin{array}{l}\text { 20mM Tris/HCl, } \mathrm{pH} 7.5,125 \mathrm{mM} \mathrm{NaCl}, 0.1 \% \\
\text { Tween-20 }\end{array}$ \\
\hline THE-buffer & $\begin{array}{l}10 \mathrm{mM} \text { Hepes, } 10 \mathrm{mM} \mathrm{KCl}, 300 \mathrm{mM} \text { trehalose } \\
\text { with or without } 0.1 \% \mathrm{BSA} \text { (filtered) }\end{array}$ \\
\hline Tris-Tricine anode buffer & $0.2 \mathrm{M}$ Tris, $\mathrm{pH} 8.9$ \\
\hline Tris-Tricine cathode buffer & $0.1 \mathrm{M}$ Tricine, $0.1 \mathrm{M}$ Tris, $0.1 \% \mathrm{SDS}, \mathrm{pH} 8.25$ \\
\hline Tris-Tricine gel buffer & $1 \mathrm{M}$ Tris, $0.1 \%$ SDS (autoclaved), $\mathrm{pH} 8.45$ \\
\hline
\end{tabular}




\subsubsection{Kits and disposables}

Table 2.3 Kits and disposables

\begin{tabular}{|c|c|}
\hline Product & Supplier \\
\hline Blotting paper & Heinemann Labortechnik \\
\hline $\begin{array}{l}\text { Complex IV Human Specific Activity } \\
\text { Microplate Assay Kit }\end{array}$ & abcam \\
\hline FastDigest restriction enzymes & Thermo Scientific \\
\hline Flexi® Rabbit Reticulocyte Lysate System & Promega \\
\hline GeneRuler DNA Ladder Mix & Fermentas \\
\hline KOD Hot Start DNA Polymerase & Novagen \\
\hline Microtube $1.5 \mathrm{~mL}$ and $2 \mathrm{~mL}$ & Sarstedt \\
\hline $\begin{array}{l}\text { MITOSOX }{ }^{\mathrm{IM}} \text { Redmitochondrialsuperoxide } \\
\text { indicator for live-cell imaging }\end{array}$ & Invitrogen \\
\hline $\begin{array}{l}\text { Pierce }{ }^{\circledR} \text { ECL Western Blotting Detection } \\
\text { Reagent }\end{array}$ & Thermo Scientific \\
\hline Pipette tips $10,200 \mu \mathrm{L}, 1 \mathrm{~mL}$ & Sarstedt \\
\hline $\begin{array}{l}\text { Precision Blue Protein }{ }^{\mathrm{IM}} \text { Standards All Blue } \\
(10- \\
250 \mathrm{kDa})\end{array}$ & BioRad \\
\hline Rapid DNA Ligation Kit & Thermo Scientific \\
\hline SP6 mMESSAGE mMACHINE® Kit & Ambion \\
\hline 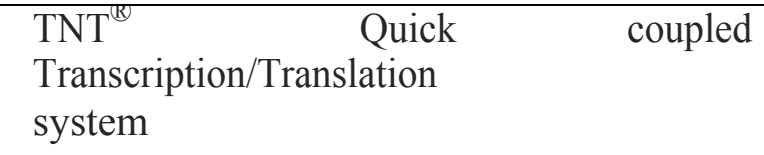 & Promega \\
\hline $\begin{array}{llll}\text { Unstained } & \text { SDS-PAGE } & \text { Protein } & \text { marker } \\
(6.5- & & & \\
200 \mathrm{kDa}) & & & \\
\end{array}$ & Serva \\
\hline $\begin{array}{l}\text { Wizard® Plus SV Gel and PCR DNA } \\
\text { Purification System }\end{array}$ & Promega \\
\hline $\begin{array}{lrlll}\text { Wizard }{ }^{\circledR} & \text { SV } & \text { Plus } & \text { Mini-Prep } & \text { DNA } \\
\text { Purification Kit } & & & \end{array}$ & Promega \\
\hline X-ray films & $\begin{array}{l}\text { Foma Bohemia (Hradec Kralove, } \\
\text { Czech Republic) }\end{array}$ \\
\hline
\end{tabular}




\subsubsection{Instruments and equipment}

Table 2.4 Instruments and equipment

\begin{tabular}{|c|c|}
\hline Instrument & Supplier \\
\hline 5415 R (centrifuge) & Eppendorf \\
\hline 5417 R (centrifuge) & Eppendorf \\
\hline 5424 (centrifuge) & Eppendorf \\
\hline 5804 R (centrifuge) & Eppendorf \\
\hline Cary 50 Bio UV-Visible Spectrophotometer & Varian \\
\hline Countess $^{\mathrm{TM}}$ automated cell counter & Thermo Scientific \\
\hline Curix 60 (developing machine) & AGFA \\
\hline Dounce homogenizer (glass-glass, BBI-8540705) & Sartorius \\
\hline EPS 601 power supply & GE Healthcare \\
\hline FACS Canto $^{\mathrm{TM}}$ II & BD Biosciences \\
\hline Fluorescence scanner FLAG-9000 & Fujifilm \\
\hline GeneAmp® PCR System 9700 (thermo cycler) & Applied Biosystems \\
\hline GD-5040 vacuum gel dryer & Scie-Plas \\
\hline Heraeus ${ }^{\circledR}$ Biofuge pico (centrifuge) & Thermo Scientific \\
\hline Heraeus ${ }^{\circledR}$ Hera cell 150 (incubator) & Thermo Scientific \\
\hline Heraeus ${ }^{\circledR}$ Hera safe (sterile hood) & Thermo Scientific \\
\hline Hoefer SE600 Ruby Blue native system & GE Healthcare \\
\hline HPTLC Silicagel60 $\mathrm{F}_{254}$ glass plates & Merck \\
\hline NanoVue ${ }^{\mathrm{TM}}$ Spectrophotometer & GE Healthcare \\
\hline Potter S (Dounce homogenizer) & Sartorius \\
\hline Semi Dry Blotting Chamber & PEQLAB Biotechnologie \\
\hline Storm $^{\mathrm{TM}} 820$ Phosphoimager & GE Healthcare \\
\hline Storage Phosphor screen & GE Healthcare \\
\hline Thermomixer comfort & Eppendorf \\
\hline Typhoon FLA 9500 Phosphoimager & GE Healthcare \\
\hline Universal 320 (centrifuge) & Hettich \\
\hline Vaccubrand $\AA 2$ C Gel pump & Scie-Plas \\
\hline VortexGenie2 & Scientific Industries \\
\hline
\end{tabular}




\subsubsection{Software}

Table 2.5 Software

\begin{tabular}{|l|l|}
\hline Software & Producer \\
\hline Microsoft Office & Microsoft \\
\hline Adobe Illustrator CS6 & Adobe \\
\hline Adobe Photoshop CS6 & Adobe \\
\hline Genious Pro & Biomatters Ltd. \\
\hline FiJi/ImageJ & OpenSource \\
\hline ImageQuant TL & GE Healthcare \\
\hline GraphPad Prism & GraphPad \\
\hline
\end{tabular}

\subsubsection{Antibodies}

Table 2.6 Primary antibodies

\begin{tabular}{|l|l|}
\hline Antibody & Source \\
\hline Rabbit polyclonal ANTI-RIESKE & Home-made \\
\hline Rabbit polyclonal ANTI-COX1 & Home-made \\
\hline Rabbit polyclonal ANTI-SDHA & Home-made \\
\hline Rabbit polyclonal ANTI-ATP5B & Home-made \\
\hline Rabbit polyclonal ANTI-LONP1 & Home-made \\
\hline Rabbit polyclonal ANTI-TIM23 & Home-made \\
\hline Rabbit polyclonal ANTI-TIM21 & Home-made \\
\hline Rabbit polyclonal ANTI-TIM22 & Home-made \\
\hline Rabbit polyclonal ANTI-COX6a & Home-made \\
\hline Rabbit polyclonal ANTI-AGK & Home-made \\
\hline Rabbit polyclonal ANTI-NDUFB8 & Home-made \\
\hline Rabbit polyclonal ANTI-TIM50 & Home-made \\
\hline Rabbit polyclonal ANTI-TOM70 & Home-made \\
\hline Rabbit polyclonal ANTI-TOM40 & Home-made \\
\hline Rabbit polyclonal ANTI-TOM20 & Home-made \\
\hline Rabbit polyclonal ANTI-COX5a & Home-made \\
\hline Rabbit polyclonal ANTI-COX4I1 & Home-made \\
\hline
\end{tabular}




\begin{tabular}{|l|l|}
\hline Rabbit polyclonal ANTI-VDAC3 & Home-made \\
\hline Rabbit polyclonal ANTI-TIM21 & Home-made \\
\hline Rabbit polyclonal ANTI-COX2 & Home-made \\
\hline Rabbit polyclonal ANTI-TACO1 & Home-made \\
\hline Rabbit polyclonal ANTI-TIM44 & Abcam, ab168649 \\
\hline Rabbit polyclonal EMRE & Santa Cruz, sc-86337 \\
\hline Rabbit polyclonal MCU & Sigma, HPA016480 \\
\hline Rabbit monoclonal MICU1 & Cell Signaling, 12524 \\
\hline Rabbit polyclonal MCUR1 & Proteintech, 24948-1 \\
\hline Rabbit polyclonal MCUb & Sigma, HPA048776 \\
\hline Rabbit polyclonal COX4I2 & Proteintech, 11463-1 \\
\hline Rabbit polyclonal CRLS1 & Abcam, ab156882 \\
\hline Rabbit polyclonal MICU2 & Abcam, ab101465 \\
\hline
\end{tabular}

Table 2.7 Secondary antibodies

\begin{tabular}{|l|l|}
\hline Antibody & Source \\
\hline Goat anti-rabbit IR880 & LI-COR, Lincoln, NE, USA \\
\hline Goat anti-mouse IR880 & LI-COR, Lincoln, NE, USA \\
\hline Goat anti-mouse HRP & Dianova, Hamburg, Germany \\
\hline Goat anti-rabbit HRP & Dianova, Hamburg, Germany \\
\hline
\end{tabular}

\subsubsection{Oligonucleotides and plasmids}

Table 2.8 siRNAs

\begin{tabular}{|l|l|}
\hline Antibody & Source \\
\hline & \\
\hline & \\
\hline & \\
\hline & \\
\hline & \\
\hline
\end{tabular}

Table 2.9 Primers 


\begin{tabular}{|l|l|}
\hline Antibody & Source \\
\hline & \\
\hline & \\
\hline & \\
\hline & \\
\hline & \\
\hline
\end{tabular}

Table 2.10 Plasmids

\begin{tabular}{|c|c|c|c|c|}
\hline Plasmid name & Purpose & Features & Marker & Reference \\
\hline pTNT & $\begin{array}{l}\text { Vector for in vitro } \\
\text { Transcription and } \\
\text { translation }\end{array}$ & $\begin{array}{l}\text { SP6, } \quad \mathrm{T7} \\
\text { promoter }\end{array}$ & Amp & Promega \\
\hline pGEM4Z & $\begin{array}{l}\text { Vector for in vitro } \\
\text { Transcription and } \\
\text { translation }\end{array}$ & $\begin{array}{l}\text { SP6, } \quad \mathrm{T7} \\
\text { promoter }\end{array}$ & Amp & Promega \\
\hline pcDNA5/FRT/TO & $\begin{array}{l}\text { Genomic } \\
\text { integration } \\
\text { vector for } \\
\text { tetracycline- } \\
\text { inducible } \\
\text { expression of } \\
\text { recombinant } \\
\text { proteins in } \\
\text { mammalian cells }\end{array}$ & $\begin{array}{l}\text { CMV } \\
\text { promotor }\end{array}$ & $\begin{array}{l}\text { Amp, } \\
\text { Hygro } \\
\text { mycin }\end{array}$ & $\begin{array}{l}\text { Thermo } \\
\text { Fisher } \\
\text { Scientific }\end{array}$ \\
\hline $\begin{array}{l}\text { pcDNA5/FRT/TO- } \\
\text { MCU-FLAG } \\
\text { (human) }\end{array}$ & $\begin{array}{l}\text { Electroporation } \\
\text { into MEF cells for } \\
\text { transient } \\
\text { expression }\end{array}$ & $\begin{array}{l}\text { CMV } \\
\text { promotor }\end{array}$ & $\begin{array}{l}\text { Amp, } \\
\text { Hygro } \\
\text { mycin }\end{array}$ & Jan Dudek \\
\hline $\begin{array}{l}\text { pcDNA5/FRT/TO- } \\
\text { TAZ-FLAG isoform } \\
1 \text { (mouse) }\end{array}$ & $\begin{array}{l}\text { Electroporation } \\
\text { into MEF cells for } \\
\text { transient } \\
\text { expression }\end{array}$ & $\begin{array}{l}\text { CMV } \\
\text { promotor }\end{array}$ & $\begin{array}{l}\text { Amp, } \\
\text { Hygromy } \\
\text { cin }\end{array}$ & $\begin{array}{l}\text { Arpita } \\
\text { Chowdhury }\end{array}$ \\
\hline $\begin{array}{l}\text { pcDNA5/FRT/TO- } \\
\text { TAZ-FLAG isoform } \\
2 \text { (mouse) }\end{array}$ & $\begin{array}{l}\text { Electroporation } \\
\text { into MEF cells for } \\
\text { transient } \\
\text { expression }\end{array}$ & $\begin{array}{l}\text { CMV } \\
\text { promotor }\end{array}$ & $\begin{array}{l}\text { Amp, } \\
\text { Hygromy } \\
\text { cin }\end{array}$ & $\begin{array}{l}\text { Arpita } \\
\text { Chowdhury }\end{array}$ \\
\hline OTC & $\begin{array}{l}\text { Vector for in vitro } \\
\text { Transcription and } \\
\text { Translation of OTC }\end{array}$ & $\begin{array}{l}\text { SP6, } \quad \mathrm{T7} \\
\text { promoter }\end{array}$ & Amp & $\begin{array}{l}\text { Mick et al., } \\
2012\end{array}$ \\
\hline
\end{tabular}




\begin{tabular}{|l|l|l|l|l|}
\hline $\begin{array}{l}\text { pGEM4Z-MCU } \\
\text { (human) }\end{array}$ & $\begin{array}{l}\text { Vector for in vitro } \\
\text { Transcription and } \\
\text { Translation of } \\
\text { MCU }\end{array}$ & $\begin{array}{l}\text { SP6, T7 } \\
\text { promoter }\end{array}$ & Amp & Jan Dudek \\
\hline $\begin{array}{l}\text { pGEM4Z-EMRE } \\
\text { (human) }\end{array}$ & $\begin{array}{l}\text { Vector for in vitro } \\
\text { Transcription and } \\
\text { Translation of } \\
\text { EMRE }\end{array}$ & $\begin{array}{l}\text { SP6, T7 } \\
\text { promoter }\end{array}$ & Amp & Jan Dudek \\
& & & \\
\hline
\end{tabular}

\subsection{Methods}

\subsubsection{Mammalian cell culture techniques}

\subsubsection{Cultivation of Mouse Embryonic Fibroblasts (MEF cells)}

H-ras transformed and SV40-large T immortalized Mouse Embryonic Fibroblasts (MEF cells) were cultured in 10\% fetal bovine serum (FBS) (GIBCO, Invitrogen) containing DMEM (Dulbecco's Modified Eagle Medium) at 5\% $\mathrm{CO}_{2}$ humidified atmosphere and $37{ }^{\circ} \mathrm{C}$. For cell passaging, MEF cells were washed once with PBS and detached from flask surface with Trypsin/EDTA for $3 \mathrm{~min}$. Trypsin was inactivated by adding an appropiate amount of FBS-containing DMEM. Cells were centrifuged at $1500 \mathrm{rpm}$ for $5 \mathrm{~min}$. at RT. Subsequently, the cell pellet was resuspended in 5-10 $\mathrm{ml}$ DMEM and 0,5-1 $\mathrm{ml}$ cell suspension were seeded to a new flask for maintenance.

\subsubsection{Silencing of mRNA/protein expression levels via RNAi}

MEF cells were seeded and grown in DMEM w/o antibiotics until a confluency of 50$80 \%$ to ensure a proliferating metabolic state. Cells were harvested and counted by Neubauer counting chamber. In between, siRNA (Eurogentec)/Lipofectamine (RNAiMax)/Optimem mix was prepared in a disposable culture tube. The final concentration of siRNA in culture was $50 \mathrm{nM}$. Therefore, the cells-to-siRNA ratio was prepared as follows: $600.000 \quad$ cells/27 $\quad \mu 1 \quad$ siRNA $\quad(20 \mu M) / 18 \quad \mu 1$ 
Lipofectamine/2,24 $\mathrm{ml}$ Optimem. The siRNA/Lipofectamine/Optimem mix was mixed by pipetting for 10 times preventing bubbles and vortexing and incubated for 20 min. at RT. The desired number of MEF cells was added to the siRNA/Lipofectamine/Optimem mix and completed with DMEM ad $10 \mathrm{ml}$. Nontarget siRNA and Targetgen siRNA were seeded to $10 \mathrm{~cm}$ plates and cultivated at $5 \%$ humidified atmosphere and $37^{\circ} \mathrm{C}$ for 3 days.

\subsubsection{Electroporation of plasmid DNA}

Plasmid DNA was transiently transfected to cultured MEF cells by electroporation via the NEON transfection system (Invitrogen). MEF cells required to be splitted one day in advance to obtain a cell confluency of $60-80 \%$. Cell detachment and harvesting was performed as described in 2.2.1.1. The cell number was determined by Neubauer counting chamber. 100.000 cells each were aliquoted to $1,5 \mathrm{ml}$ Eppendorf tubes and centrifuged at $2000 \mathrm{rpm}, 5 \mathrm{~min}$. Afterwards, cells were washed with PBS and centrifuged again. The cell pellet is resuspended in R-buffer from the kit and mixed with $1 \mu \mathrm{g}$ plasmid DNA via pipetting up and down mildly. The cell/plasmid DNA mix was then electroporated at these conditions: Program HEKB, Voltage 1150, Width 20, pulses 2. Electroporated cells were transferred immediately to prepared, warm DMEM medium and seeded in appropiate plates for growing. After 2-3 days cells were harvested at a confluency of $80-100 \%$.

\subsubsection{Control and patient-derived inducent pluripotent stem cell cardiomyocytes (iPSC-CM)}

Control and patient-derived inducent pluripotent stem cell cardiomyocytes (iPSCCMs) were generated and characterized as already described (Dudek et al., 2016). The iPSC-CM line TAZ 10 was obtained from a BTHS patient, which carried the TAZ mutation c.590 G > T (Dudek et al., 2013). The iPSC-CMs were seeded as adherent cultures on Geltrex-coated cell culture plates in E8 Essential medium (Life Technologies). iPSCs were differentiated to cardiomyocytes within 30 days via modulation of canonical Wnt pathway. Addition of $4 \mathrm{mM}$ lactate in substitution of glucose for 6 days caused enrichment of cardiomyocytes (Tohyama et al., 2013). 
iPSC-CMs were cultivated in RPMI 1640 medium (HEPES/GlutaMax, Life technologies) supplemented with insulin and B27 (Life technologies). iPSC-CMs were detached from dishes by $0,25 \%$ trypsin-EDTA (Life technologeis) and reseeding.

\subsubsection{Mouse model}

\subsubsection{Maintenance of mice}

The study and the maintenance of all mice were conducted in accordance with the German Animal Welfare Act and confirmed by the Landesamt für Verbraucherschutz und Lebensmittelsicherheit Niedersachsen, Germany (AZ: 33.9-42502-04-15/1991). WT C57BL/6J and transgenic mice (ROSA26H1/tetO-shRNA:TAZ) were feeded by standard rodent chow and administration of doxycyline $(625 \mathrm{mg} / \mathrm{kg}$ ) (Acehan et al., 2011; Soustek et al., 2011). Doxycycline treatment was annuled during mating to prevent infertility oft he males. Females were only treated with doxycyline until one week before mating. Doxycycline treatment was proceeded when copulatory plugs were noticed. The pups were treated for 8 weeks continuously. Assessment oft he genotype was performed using PCR (Acehan et al., 2011).

\subsubsection{Mitochondria isolation from mouse tissues via differential centrifugation}

Mouse tissues (heart, skeletal muscle, brain, liver) were dissected and cut in small pieces by dissection scissors. The minced organ pieces were resuspended with $2 \mathrm{ml}$ of mouse tissue isolation buffer (Table 2.2) and dounced with 20 gentle handstrokes in a glass-glass Dounce homogenizer (Sartorius). The potter was kept on ice. The suspension was centrifuged at $1000 \mathrm{~g}, 10 \mathrm{~min} ., 4{ }^{\circ} \mathrm{C}$., supernatant was kept and again centrifuged to discard remnant insolved material. Transfer of suspension was performed on a mild way with plastic Pasteur pipettes or decanting. Cleared supernatant was spinned down at $10.000 \mathrm{~g}, 10 \mathrm{~min} ., 4{ }^{\circ} \mathrm{C}$ to obtain pelleted mitochondrial fraction. Mitochondrial pellet was resuspendend with 50-200 $\mu$ l mouse tissue isolation buffer receiving an end concentration of around $10 \mu \mathrm{g} / \mu \mathrm{l}$. 


\subsubsection{Molecular biology}

\subsubsection{Transformation of plasmid into E.coli}

The ligated plasmid of previous cloning was transformed into bacteria to amplify the yield. Therefore, rubidium-competent bacteria (Escherichia coli XL-1 blue) were thawed on ice. $50 \mu \mathrm{l}$ of bacteria were mixed with $5 \mu \mathrm{l}$ plasmid and incubated on ice for $15 \mathrm{~min}$. After that, the plasmid/bacteria mix was heatshocked at $42{ }^{\circ} \mathrm{C}$ for $2 \mathrm{~min}$. Mix was put on ice for $2 \mathrm{~min}$ and $1 \mathrm{ml} \mathrm{LB}$ medium (lysogeny broth) was added and incubated at $37{ }^{\circ} \mathrm{C}$ for 1 hour. Bacteria were centrifuged at full speed for 1 min and pellet was resuspended in $100 \mu \mathrm{LB}$ medium. The resuspension was plated on a Ampicillin (0,1 g/l)-resistent LB agar (15 g/ml agar) and incubated over night at 37 ${ }^{\circ} \mathrm{C}$. On the next day clones were picked by a yellow pipette tip and inoculated into 5 ml LB medium (Ampicillin-resistent). Bacteria grew over night at $37{ }^{\circ} \mathrm{C}$ under constant shaking and next day the bacteria suspension was either reinoculated into $100 \mathrm{ml} \mathrm{LB}$ medium to reach higher yield of plasmid (Maxiprep) or plasmid was purified on a lower scale (Miniprep) to examine the insert sequence by sequencing or plasmid digestion.

\subsubsection{Plasmid DNA isolation from $E$.coli}

Plasmid isolation on a larger scale (Maxiprep) was used to reach plasmid concentrations of $5 \mu \mathrm{g} / \mu \mathrm{l}$, which was nessecary for electroporation of plasmids to cells (2.2.1.3). The optical density (OD) of a $100 \mathrm{ml} \mathrm{LB}$ medium containing E. coli bacteria was measured. The bacteria suspension should have a OD of 2,3 - 2,6, otherwise the suspension was diluted with LB medium. Culture was pelleted in $50 \mathrm{ml}$ falcon tubes at $3400 \mathrm{~g}$ for $10 \mathrm{~min}$ and the supernatant was discarded. The Maxiprep was perfromed with the ZymoPURE Plasmid Maxiprep kit. Bacteria pellet was resuspended with $14 \mathrm{ml}$ of ZymoPURE P1 resuspension and lysing buffer and mixing by inverting six times. Then, $14 \mathrm{ml}$ of ZymoPure P2 buffer were added, gently mixed but thoroughly by inversion. ZymoPure P3 neutralizaiton buffer was added (14 ml) and mixed by inversion. It took 6-8 min to reach precipitation of the suspension. The suspension was transferred to a $50 \mathrm{ml}$ syringe. The suspension was pushed through a 
filter by a plunger into a falcon tube to clear the solution from precipitations. $14 \mathrm{~mL}$ ZymoPURE Binding Buffer was added to the cleared lysate and mixed by inverting several times. The ZymoSpin Column reservoir was assembled onto a vacuum manifold. The lysate was added to the ZymoSpin Column reservoir and vacuum was turned on causing, that all of liquid has passed completely through the column. The upper part of the reservoir was removed from the column assembly and $5 \mathrm{ml}$ of ZymoPURE Wash 1 buffer was added. Vacuum was kept on and washing buffer passed through the column. The washing step was repeated twice with $5 \mathrm{ml}$ of ZymoPURE Wash 2 buffer. Column was transfered to a table-top centrifuge. Residual washing buffer was removed by centrifugation (10000g for $1 \mathrm{~min})$. Column was transfered to a clean $1,5 \mathrm{ml}$ tube and kept for $2 \mathrm{~min}$ at RT. $250 \mu 1$ pre-warmed deionizied water were added slowly to the column. Column was incubated for $2 \mathrm{~min}$ at RT and centrifuged at $10000 \mathrm{~g}$ for $1 \mathrm{~min}$. The plasmid concentration was determined and the concentration was adjusted to $5 \mu \mathrm{g} / \mu \mathrm{l}$. If the concentration was too low, the plasmid solution was subjected to a SpeedVac to evaporate water and concentrate the plasmid.

Plasmid isolations from E. coli were performed on a smaller scale (Miniprep) as well. Therefore, PureYield Plasmid Miniprep System kit by Promega was used and was followed by manufacturers instructions.

\subsubsection{Polymerase chain reaction (PCR)}

DNA fragments from purified plasmids were amplified by the KOD Hot Start DNA Polymerase kit. Manufacturers instructions were followed. The reaction mixture had a total volume of $50 \mu \mathrm{l}$ and contained $1 \mathrm{x}$ KOD reaction buffer, $0.3 \mu \mathrm{M}$ forward and reverse primer pairs, $0.2 \mathrm{mM}$ dNTPs, plasmid or cDNA (template), $1 \mathrm{mM} \mathrm{MgSO}_{4}$, $0.2 \mathrm{mM}$ dNTPs, and 1 unit of KOD DNA polymerase. The PCR started with a heating step $\left(2 \mathrm{~min}\right.$ at $\left.95^{\circ} \mathrm{C}\right)$ and 35 cycles followed. The heating step of the PCR cycle was $95^{\circ} \mathrm{C}$ for $10 \mathrm{sec}$. The time and temperature of the annealing step was depending on the primer pairs $\left(10-15 \mathrm{sec}\right.$. at $\left.52^{\circ}-55^{\circ} \mathrm{C}\right)$. Elongation step took $20-100 \mathrm{sec}$. at $70^{\circ} \mathrm{C}$ and was depending on the PCR product size.

\subsubsection{Agarose gel electrophoresis}


Agarose gel electrophoresis was performed to confirm the presence of a specific PCR product and to examine the correct size after the PCR. The separation of DNA was conducted in a $1 \%$ agarose gel. Therefore, dry agarose was dissolved in $1 \mathrm{x}$ TAE buffer (Table 2.2). The agarose solution was heated in a microwave and colled under gentle shaking. One drop of DNA intercalating ethidium bromide was added and solution was casted in the gel apparature. After solidification of the agarose gel $5 \mu \mathrm{L}$ of the PCR reaction were mixed with $5 \mu \mathrm{L}$ of $2 \mathrm{x}$ loading dye. The samples and the ladder mix (Gene Ruler) were loaded into the slots of the gel and run for $20 \mathrm{~min}$ at 120 V. UV-light visualized the DNA fragments.

\subsubsection{Purification of PCR products}

The Wizard ${ }^{\circledR}$ Plus SV Gel and PCR DNA Purification kit was used to purify PCR products according to manufacturer's instructions. PCR products were either dissolved from gel slices after agarose gel electrophoresis or purification was performed with the PCR solution directly after amplification. Following electrophoresis, DNA band of interest was excised from gel and gel slice was placed in a 1,5 ml microcentrifuge tube. $10 \mu \mathrm{l}$ of membrane binding buffer per $10 \mathrm{mg}$ of gel slice were added. The solution was incubated at $50-65^{\circ} \mathrm{C}$ under harsh agitation until the gel slice was completely dissolved. For the second variant, equal amount of membrane binding buffer was added to the $50 \mu$ of PCR product. The SV Minicolumn was inserted into the Collection Tube. Prepared PCR product or dissolved gel mixture were transferred to the Minicolumn assembly and incubated for $1 \mathrm{~min}$ at RT. Minicolumn was centrifuged at $16000 \mathrm{~g}$ for $1 \mathrm{~min}$ and flowthrough was discarded. Minicolumn was inserted into the Collection Tube. To wash the column, $700 \mu \mathrm{l}$ Membrane Wash Solution were added and centrifuged at $16000 \mathrm{~g}$ for $1 \mathrm{~min}$. This washing step was repeated with $500 \mu \mathrm{l}$ Membrane Wash Solution. Minicolumn was centrifuged at $16000 \mathrm{~g}$ for $5 \mathrm{~min}$. The Collection Tube was emptied and the column assembly was centrifuged for $1 \mathrm{~min}$ with the microcentrifuge lid open to allow evaporation of remnant ethanol. The Minicolumn was transferred to a clean 1,5 $\mathrm{ml}$ microcentrigue tube. $50 \mu 1$ of nuclease-free water were added to the Minicolumn and incubated for $1 \mathrm{~min}$ at RT. To elute the DNA from the column, it was centrifuged at $16000 \mathrm{~g}$ for $1 \mathrm{~min}$ at RT. The purified DNA was quantified by a NanoVue ${ }^{\mathrm{TM}}$ spectrophotometer and stored at $4{ }^{\circ} \mathrm{C}$. 


\subsubsection{Cloning}

Purified PCR products were inserted into plasmids. Both, PCR product and the plasmid underwent restriction digestion using Fast Digest restriction enzymes (Thermo Scientific). The total volume of the digestion of the PCR product was $30 \mu 1$. $1 \mu \mathrm{l}$ of both restriction enzymes, $5 \mathrm{ml}$ of nuclease-free water, $3 \mu 1$ of 10x Fast Digest buffer and $20 \mu \mathrm{l}$ of the PCR reaction were mixed and incubated for $30 \mathrm{~min}$ at $37{ }^{\circ} \mathrm{C}$ under mild agitation. $1 \mu \mathrm{l}$ plasmid vector was mixed with $1 \mu \mathrm{l}$ of both restriction enzymes, $2 \mu 1$ of 10x Fast Digest buffer and $15 \mathrm{ml}$ nuclease-free water. Plasmid and PCR product were digested for 1-2 hours at $37{ }^{\circ} \mathrm{C}$. The plasmid was dephosphorylated by adding alkaline phosphatase $(1 \mu 1)$ and incubation for $45 \mathrm{~min}$ at $37{ }^{\circ} \mathrm{C}$. Both samples were purified by PCR purification kit. Ligation of plasmid and PCR product was performed using the Rapid DNA Ligation Kit (Thermo Scientific). $2 \mu \mathrm{l}$ of digested plasmid, $1 \mu \mathrm{L}$ T4 DNA ligase, $60 \mathrm{ng}$ of digested PCR product and $1 \mathrm{x}$ ligation buffer were mixed to a total volume of $20 \mu$. The ligation mix was incubated for $30 \mathrm{~min}$ at $22{ }^{\circ} \mathrm{C}$ at mild agitation. The plasmid was transformed into XL Blue E. coli bacteria (2.2.3.1) and isolated from colonies (2.2.3.2). Constructs were tested by sequencing (SEQLAB, Göttingen, Germany) and restriction digestion followed by separation via agarose gel electrophoresis (2.2.3.4)

\subsubsection{In vitro transcription}

The SP6 mMESSAGE mMACHINE® Kit by Ambion was used to perform in vitro transcription from purified PCR products, which contained a SP6 promotor. Due to manufacturer's instructions, $2 \mu 1$ of 10x NTP/CAP, $10 \mu 1$ of $2 x$ reaction buffer, $6 \mu 1$ of purified PCR product (independently from the concentration) and $2 \mu \mathrm{L}$ of enzyme mix were mixed to a total volume of $20 \mu \mathrm{l}$. The transcription mix was incubated for 90 min at $37^{\circ} \mathrm{C}$ under mild agitation. $30 \mu \mathrm{L}$ of lithium chloride (7.5M and 50mM EDTA) were added to precipitate the synthesized RNA over night at $-20{ }^{\circ} \mathrm{C}$. Samples were centrifuged at $20000 \mathrm{~g}$ for $15 \mathrm{~min}$ and supernatants were removed. The pellet was resuspended with $70 \%$ ethanol and centrifuged at $20000 \mathrm{~g}$ for $15 \mathrm{~min}$. Supernatant was discarded carefully and residual ethanol was evaporated at RT. The pellet was resuspended in nuclease-free water and incubated on ice for $30 \mathrm{~min}$. The volumes 
were depending on the size of the pellet $(10-40 \mu l)$. Synthezised RNA was quantified by a NanoVueTM spectrophotometer. RNA was stored at $-80^{\circ} \mathrm{C}$.

\subsubsection{In vitro translation}

The Flexi ${ }^{\circledR}$ Rabbit Reticulocyte Lysate kit (Promega) was used to perform in vitro translation of mitochondrial precursor proteins from mRNA. Therefore, $33 \mu 1$ of Flexi ${ }^{\circledR}$ Rabbit Reticulocyte Lysate were mixed with $1 \mu \mathrm{g}$ of mRNA in a final volume of $50 \mu \mathrm{l}$. Due to manufacturer's instructions, $1 \mu \mathrm{L}$ of amino acid mixture without methionine $(1 \mathrm{mM})$, magnesium acetate $(0-2 \mathrm{mM})$, potassium chloride $(70-120 \mathrm{mM})$ and ${ }^{35} \mathrm{~S}$-methionine were added and incubated for $90 \mathrm{~min}$ at $30^{\circ} \mathrm{C}$. After that, $5 \mu 1$ of non-radioactive methionine were added for $1 \mathrm{~min}$. Sucrose was added for an additional minute. The protein lysate was kept on ice for an in-vitro precursor import into freshly purified mitochondria (2.2.5.1).

The second approach to synthesize mitochondrial precursor proteins for in vitro import reactions was a combined transcription and translation by the TNT® Quick coupled Transcription/Translation kit (Promega). Transcription and translation were performed directly from the plasmid (pTNT backbone), which contained the open reading frame of the precursor protein. $200 \mathrm{ng}$ of the plasmid were mixed with $40 \mu \mathrm{l}$ of the TNT® Quick Master Mix and $50 \mu \mathrm{Ci} 35 \mathrm{~S}-$ methionine for 90 min at $30{ }^{\circ} \mathrm{C} .5 \mu \mathrm{l}$ of non-radioactive methionine were added for $1 \mathrm{~min}$. Sucrose was added for an additional minute. The protein lysate was kept on ice for an in-vitro precursor import into freshly purified mitochondria (2.2.5.1).

\subsubsection{Protein separation and protein complex analysis}

\subsubsection{Determination of protein concentration via Bradford assay}

Protein concentrations of mitochondrial or cellular fractions were determined via Bradford assay (Roti ${ }^{\circledR}-Q u a n t$ (Serva)) to obtain equal protein amount adjustments in subsequent experiments.

The protein amount in the sample was calculated by linear regression curve of the bovine immunoglobulin standard (IgG standard). The IgG standard solution was 
diluted to $0,7.5,15,30$ and $60 \mu \mathrm{g} / \mu \mathrm{l}$ and mitochondrial/cellular suspensions were diluted $1 / 10$. Water was added to obtain a final volume of $86 \mu \mathrm{L} .1 / 5$ diluted Roti ${ }^{\circledR}$ Quant reagent was appended to all solutions and the optical density was measured at 595 nm (Cary 50 Bio UV-visible spectrophotometer (Varian)).

\subsubsection{Protein separation via SDS-PAGE analysis}

Protein samples were denatured by SDS/Laemmli sample buffer (Table 2.2) at $95{ }^{\circ} \mathrm{C}$, 5 min. under reducing conditions (w/DTT) or at $37^{\circ} \mathrm{C}, 15 \mathrm{~min}$. under non-reducing conditions (w/o DTT). Denatured protein samples were applied to 10-16,5\% SDSPAGE (Laemmli, 1970) to separate proteins electrophoretically according to their molecular weight.

The $10-16,5 \%$ running gel was prepared by $386 \mathrm{mM}$ Tris/ $\mathrm{HCl} \mathrm{pH} 8.8,0.1 \%$ SDS, $30 \% / 0.8 \%$ acrylamide/bisacrylamide solution (Roth). The stacking gel was prepared by $80 \mathrm{mM}$ Tris $/ \mathrm{HCl}, \mathrm{pH} 6.8$ and $0.1 \%$ SDS, 4\% acrylamide. APS and TEMED initiated and catalyzed acrylamide polymerization. Denatured protein samples were loaded to polymerized stacking gel wells and were electrophoretically separated at $250 \mathrm{~V}, 25 \mathrm{~mA} / \mathrm{gel}, 2-3$ hours in $1 \mathrm{x}$ SDS running buffer (GE Healthcare, Electrophoresis Power Supply EPS 601).

\subsubsection{Tris-Tricine-SDS-PAGE}

Tris-Tricine polyacrylamide gradient gels (10-18\%) were utilized to improve the separation of the whole molecular weight range and to increase the resolution of small proteins with a size lower than $15 \mathrm{kDa}$. 10-18\% Tris-Tricine-SDS-PAGE resolving gels was prepared by $1 \mathrm{M}$ Tris/ $\mathrm{HCl} \mathrm{pH} 8.45,49.5 \% / 3 \%$ acrylamide/bis-acrylamide, $0.1 \%$ SDS and $13 \%$ glycerol (for $18 \%$ ). $10 \%$ and $18 \%$ solutions were casted with a homemade gradient mixer. The $4 \%$ stacking gel comprised $1 \mathrm{M}$ Tris $/ \mathrm{HCl}, 4 \%$ acrylamide, $\mathrm{pH} 8.45$ and $0.1 \%$ SDS and was casted on top of the resolving gradient gel. APS and TEMED initiated and catalyzed acrylamide polymerization. Denatured protein samples were loaded to polymerized stacking gel wells and were electrophoretically separated at $80 \mathrm{~V}, 25 \mathrm{~mA} / \mathrm{gel}$, overnight in Tris-Tricine cathode and anode buffer (Table 2.2) (GE Healthcare, Electrophoresis Power Supply EPS 601). 


\subsubsection{Blue Native Polyacrylamid Gel Electrophoresis (BN-PAGE)}

Native protein complex separation was performed by Blue Native Polyacrylamide Gel Electrophoresis (BN-PAGE) (Schägger \& Jagow, 1991; Dekker et al., 1996) in a gradient gel system (4-13\%). Resolving gradient gel solution (13\%) was composed of acrylamide/bis-acrylamide (49.5\%/3\%), 66.67mM 6-aminocaproic acid, 50mM BisTris/HCl pH 7.0 and $20 \%$ glycerol. Stacking gel solution contained 4\% acrylamide/bis-acrylamide (49.5\%/3\%), 66.67mM 6 -aminocaproic acid and 50mM Bis-Tris/ $\mathrm{HCl} \mathrm{pH}$ 7.0. BN resolving gels were casted in a homemade gradient-mixer and polymerized with TEMED and APS.

Isolated mitochondrial fractions and cell lysates were spun down $(10,000 \mathrm{x} \mathrm{g}, 5 \mathrm{~min}$, $4{ }^{\circ} \mathrm{C}$ ) and the pellet was resuspended in 1\%-digitonin-containing solubilization buffer (Table 2.2) by pipeting 20 times, vigorously. The end concentration was $1-2 \mu \mathrm{g}$ protein $/ \mu \mathrm{L}$ solubilization buffer depending on how much amout of protein was required to detect certain proteins by western blotting. Mitochondrial/cellular fractions were incubated on ice for $20 \mathrm{~min}$. and mixed slightly every $5 \mathrm{~min}$. The following centrifugation step $\left(20,000 \mathrm{x} \mathrm{g}, 10 \mathrm{~min}, 4^{\circ} \mathrm{C}\right)$ cleared the solubilized mitochondrial/cellular micelles from insoluble material. 10x Blue-Native Loading dye (Table 2.2) was added to the supernatant, which were centrifuged for a last clearing step $\left(20,000 \mathrm{x} \mathrm{g}, 3 \mathrm{~min}, 4^{\circ} \mathrm{C}\right)$. Depending on the proteins of interest, which were supposed to be detected by western blotting, from $25 \mu \mathrm{g}$ (respiratory chain complexes) up to $200 \mu \mathrm{g}$ (MCU-containing complexes) were loaded to each BNPAGE slot.

Protein complex separation via BN-PAGE was performed in a cooled tank (SE600 Ruby, Hoefer, Amersham Biosciences). BN-PAGE gels were run at $4{ }^{\circ} \mathrm{C}$ first at $400 \mathrm{~V}$ and $15 \mathrm{~mA} /$ gel for 1,5 hours and Coomassie Brilliant Blue G-250-containing cathode buffer was exchanged with cathode buffer without Coomassie Brilliant Blue G-250. Following this, BN-PAGE gel was running at $100 \mathrm{~V}$ and $15 \mathrm{~mA} / \mathrm{gel}$ overnight.

\subsubsection{2D-BN-SDS-PAGE analysis}

After native protein complex separation in BN-PAGE (2.2.4.4) single BN stripes were cut out and assembled into a denaturating SDS-PAGE apparatus to separate and 
detect proteins of interest, which were not detectable in first $\mathrm{BN}$ dimension via Western Blot analysis. As described in 2.2.4.4 mitochondrial or cellular fractions were solubilized and separated on BN-PAGE. Completely cutted single BN lanes were denatured in 1\% DTT-containing 1x SDS running buffer for $30 \mathrm{~min}$. at RT. The BN lane was assembled into glass plates for a regular-sized SDS-PAGE gels (2.2.4.2). Base gel and afterwards the SDS resolving gel solution (16,5\%) were casting as described above (2.2.4.2). After running gel polymerization the $\mathrm{BN}$ stripe was implemented into the SDS stacking gel (4\%). A two-well comb was added to stacking gel to gain two lanes for the molecular weight marker and the input control. Run $16,5 \%$-SDS-PAGE at $80 \mathrm{~V}, 25 \mathrm{~mA} / \mathrm{gel}, 12$ hours.

\subsubsection{Western Blotting and Immunodetection}

Semi-dry blotting chambers by PEQLAB (Biotechnologie $\mathrm{GmbH}$ ) were used to transfer proteins and protein complexes after electrophoretic separation in polyacrylamide gels to Polyvinylidene fluoride (PVDF) membranes (Merck). Those were activated by methanol and washed with 1x blotting buffer (Table 2.2). Both, the polyacrylamide gel and the PVDF membrane were assembled with filter papers (Heinemann Labortechnik) soaked in 1x blotting buffer and incorporated into blotting chamber. SDS-PAGE gels were transfered at 25V, $250 \mathrm{~mA}, 2.5$ hours and BN-PAGE gels at $25 \mathrm{~V}, 400 \mathrm{~mA}, 3$ hours.

Afterwards, the PVDF membrane was stained with Coomassie Brilliant Blue solution (Table 2.2) for $3 \mathrm{~min}$. and destained for $5 \mathrm{~min}$. to visualize Coomassie-bound protein bands. The membrane was cut according to the molecular size of the proteins to be analysed for immunodetection. Methanol was utilized to destain and reactivate the membrane to prevent interference with immunodetection. The membrane was blocked for at least 2 hours at RT in skimmed milk (10\% milk powder in 1x TBS-T (Table 2.2)). After blocking, membrane stripes underwent primary antibody decoration overnight at $4{ }^{\circ} \mathrm{C}$. Homemade antibodies were diluted in $5 \%$ skimmed milk and commercial antibodies were hosted by $1 \mathrm{x}$ TBS-T (Table 2.6). Membranes were mildly washed in 1x TBS-T three times at RT for $10 \mathrm{~min}$. Horseradish peroxidaseconjugated seecondary antibody (Table $2.7,1 / 5000$ or $1 / 10000$ in $5 \%$ skimmed milk) was added to the membrane for 1 hour at RT. The membrane was washed three times in TBS-T at RT. For immunodetection membranes were incubated with Pierce ${ }^{\circledR}$ ECL 
Western Blotting Detection Reagent (Thermo Scientific) and exposed to X-ray films. These X-ray films were developed by

\subsubsection{Autoradiography}

Digital autoradiography was used to visualize and to assess radioactivly labeled proteins. Radioactive samples were separated via electrophoresis and blotted to PVDF membranes as described above (2.2.4.6). The membrane was stained and destained with Coomassie and destainer solution (Table 2.2) for several minutes and dried by a hairdryer. The molecular weight marker was marked with radioactive ink and was sellotaped twice. Radioactive signals were exposed for at least one day to storage phosphor screens (GE Healthcare). A Storm820 scanner scanned and digitalized the radioactive signals, which were quantified by ImageJ software.

\subsubsection{Mitochondria}

\subsubsection{Crude isolation of mitochondrial fractions from mammalian cells via differential centrifugation}

MEF cells were seeded and harvested at a confluency of 80-100 \% 1-3 days later. In advance, $50 \mathrm{ml}$ falcon tubes (Sarstedt) were weighed to determine the cell pellet weight later. Medium in $20 \mathrm{~cm}$ plates were discarded and washed with PBS once. 5 $\mathrm{ml}$ PBS per plate was added and cells were scraped with rubber policeman and transferred to $50 \mathrm{ml}$ falcon tubes to be centrifuged at $400 \mathrm{~g}, 5 \mathrm{~min}$., $4{ }^{\circ} \mathrm{C}$. Cell pellets were resuspendend in cold Isotonic buffer (Table 2.2) and centrifuged at 1000g, 5 $\min , 4{ }^{\circ} \mathrm{C}$. Supernatants were carefully pipetted off and cell pellets were weighed. Cells were resuspended in Hypotonic buffer (Table 2.2) with plastic Pasteur pipettes ( $5 \mathrm{ml} / \mathrm{g}$ cells).

Cells were homogenized gently with 10 handstrokes by glass-glass Dounce homogenizer (Sartorius). Cells were allowed to swell for 5-7 minutes to release mitochondria upon hypotonic conditions. Hypertonic buffer (Table 2.2) was added $(1,1 \mathrm{ml} / \mathrm{g}$ cells $)$ to obtain isotonic conditions and one volume of BSA-containing Isotonic buffer was added. Cell suspension was centrifuged at $1000 \mathrm{~g}, 10 \mathrm{~min}, ., 4{ }^{\circ} \mathrm{C}$ 
and mitochondria-enriched supernatant was centrifuged again to discard residual unsolved material. Mitochondrial fractions were gained by a $10.000 \mathrm{~g}$ step. Supernatant was discarded and mitochondrial pellet was resuspended with 50-200 $\mu 1$ Isotonic buffer receiving an end concentration of around $10 \mu \mathrm{g} / \mu \mathrm{l}$.

\subsubsection{Carbonate extraction}

Carbonate extraction was used to determine the topology of mitochondrial membrane proteins. Carbonate solutions ( $\mathrm{pH} \mathrm{10,8}$ and $\mathrm{pH} 11,5)$ were made freshly. Three times $60 \mu \mathrm{g}$ mitochondria were pelleted at $10000 \mathrm{~g}, 5 \mathrm{~min} ., 4{ }^{\circ} \mathrm{C}$ and resuspended in three different solutions, respectively. First $60 \mu \mathrm{g}$ of mitochondria were resuspended in 100 $\mu \mathrm{l} \mathrm{pH} 10,8$ carbonate solution and second $60 \mu \mathrm{g}$ in $100 \mu \mathrm{pH} 11,5$ carbonate solution. Third $60 \mu \mathrm{g}$ mitochondria were resuspended in $100 \mu 10,1 \%$ Triton X-100 solution. All incubations were on ice for $20 \mathrm{~min}$. All suspensions were centrifuged at $100.000 \mathrm{~g}$, 45 min., $4{ }^{\circ} \mathrm{C}$. Supernatants were divided from pellets and pellets were resuspenden in $100 \mu \mathrm{l}$ SEM buffer. Proteins from all solutions were precipitated by TCA precipitation (trichloroacetic acid) for $30 \mathrm{~min}$. on ice. Therefore $21 \mu \mathrm{L} 72 \%$ TCA were added at RT to gain 17\% TCA solution, samples were vortexed and incubated for $30 \mathrm{~min}$. . Precipitates were centrifuged at $20000 \mathrm{~g}$ for $30 \mathrm{~min}$. at $4{ }^{\circ} \mathrm{C}$ and pellets were resuspended with 2x Laemmli buffer and resolved via SDS-PAGE analysis (2.2.4.2).

\subsubsection{Submitochondrial localization studies by swelling of mitochondria}

$100 \mu \mathrm{g}$ of pelleted mitochondria were resuspended in $100 \mu \mathrm{l}$ of isotonic SEM buffer to maintain mitochondrial membranes. This was was aliquoted to $3 \times 30 \mu 1$. In parallel, $100 \mu \mathrm{g}$ of pelleted mitochondria were gently resuspended in hypotonic EM buffer to swell mitochondria, disrupt the outer membrane and gain mitoplasts. Mitochondrial solutions in isotonic as well as in hypotonic buffer were incubated on ice for $25 \mathrm{~min}$.. Afterwards, proteinase $\mathrm{K}(\mathrm{PK})$ was added to the three aliquots per treatment in different amounts $(0,5$ and $10 \mu \mathrm{l})$. PK had access to inner mitochondrial membrane localized proteins, whereas PK could only degrade outer mitochondrial proteins in SEM resuspended intact mitochondria. Meanwhile, additional $60 \mu \mathrm{g}$ mitochondria were resuspended in $60 \mu \mathrm{l} \mathrm{EM}$ buffer. $10 \mu \mathrm{PK}$ was added to one of those samples 
and both samples were sonicated for 20 seconds each. Sonication generated micelles from mitochondria causing complete access of PK to mitochondrial proteins of all compartments. All samples were PK-treated for 10 min. on ice and samples were centrifuged at $13000 \mathrm{~g}$ at $4{ }^{\circ} \mathrm{C}$. Mitochondrial pellets were resuspended in $1 \mathrm{x}$ Laemmli buffer. Samples were boiled at $95{ }^{\circ} \mathrm{C}$ for $10 \mathrm{~min}$. to inactivate PK activity. Denatured samples should be loaded and resolved on SDS-PAGE immediately (2.2.4.2).

\subsubsection{FLAG immunoprecipitation}

FLAG immunoprecipitation (FLAG-IP) was performed from whole cells or from isolated mitochondria. FLAG-tagged proteins were transiently transfected to MEF cells via electroporation (2.2.1.3) and FLAG-IP was performed 3 days later. A untransfected WT control was seeded in parallel as a negative control of the FLAGIP. Purified mitochondria or cell suspensions were centrifuged at $10000 \mathrm{~g}$ for $5 \mathrm{~min}$. The amount of mitochondria was dependeing on the purpose of the IP and could range between $0,5 \mathrm{mg}$ and $5 \mathrm{mg}$ of mitochondria or cell lysates. The supernatant was removed and the pellet was resuspended in $200 \mu$ solubilization buffer (final: $50 \mathrm{mM}$ Tris/HCl, $50 \mathrm{mM} \mathrm{NaCl}, 10$ \% Glycerol, 5 mM EGTA, 1 \% Digitonin, $200 \mu \mathrm{M}$ PMSF) by pipetting 20 times up and down. $600 \mu \mathrm{L}$ of solubilization buffer was added and again mixed by pipetting for 10 times. Lysates were incubated on ice for $30 \mathrm{~min}$. and centrifuged at $20000 \mathrm{~g}$ for $10 \mathrm{~min}$ at $4^{\circ} \mathrm{C}$. Unsolubilized debris was removed. Supernatant was taken and added to a prepared mobicol column (M2 Affinity Matrix (Sigma), 4G). To equilibrate the column, $40 \mu 1$ FLAG beads slurry were added to the mobicol column and washed 3 times by adding washing buffer (final: $50 \mathrm{mM}$ Tris/HCl, $50 \mathrm{mM} \mathrm{NaCl}, 10 \%$ Glycerol, 5 mM EGTA, 0,3\% Digitonin, $200 \mu \mathrm{M}$ PMSF) and centrifugation at $100 \mathrm{~g}, 30 \mathrm{sec}$. . The lysate was incubated with the FLAG beads for 1 hour at $4{ }^{\circ} \mathrm{C}$ on a rotating wheel. Afterwards, unbound material was centrifuged at $200 \mathrm{~g}, 1 \mathrm{~min}$. . FLAG beads were washed 5 times with $500 \mu \mathrm{l}$ washing buffer (centrifugation steps $100 \mathrm{~g}, 30 \mathrm{sec}, 4{ }^{\circ} \mathrm{C}$ ). To elute proteins interacting with FLAG beads, $40 \mu \mathrm{g}$ FLAG peptides (180 $\mu \mathrm{l}$ washing buffer and $20 \mu \mathrm{l}$ FLAG peptides) were added to the column and incubated for $30 \mathrm{~min}$. at $4{ }^{\circ} \mathrm{C}$. Lysate were centrifuged for $1 \mathrm{~min}$. at $200 \mathrm{~g}$. Elution was mixed with SDS- or BN-PAGE loading dye (Table 2.2), depending on the aim of the experiment and loaded on SDS- (2.2.4.2) 
or BN-PAGE (2.2.4.4) for further separation.

\subsubsection{Antibody coating to Protein-A-Sepharose (PAS)}

To address endogenous protein-protein interactions, co-immunoprecipitations (Co-IP) were performed with antibodies (raised in rabbit) coated to Protein-A-Sepahrose (PAS). Therefore, $75 \mu 150 \%$ PAS were added to mobicol column (M2 Affinity Matrix (Sigma), 4G) and washed twice with $500 \mu 10.1 \mathrm{M} \mathrm{KPi} \mathrm{pH} \mathrm{7,4} \mathrm{by} \mathrm{centrifuging}$ at $100 \mathrm{~g}$ for $30 \mathrm{sec}$. In parallel, $150 \mu \mathrm{l}$ antibody serum were diluted in $150 \mu \mathrm{KPi}$ solution. PAS beads and antibodies were incubated for 1 hour at RT while mixing on a rotating wheel. The crosslinker dimethyl pimelimidate (DMP) was thawed and a 5 $\mathrm{mg} / \mathrm{ml}$ solution in $0,1 \mathrm{M}$ sodium borate $\mathrm{pH}$ 9,0 was prepared. The column was washed twice with $0.1 \mathrm{M}$ sodium borate and $350 \mu \mathrm{l}$ of the DMP solution was added. Beads and DMP were incubated for $30 \mathrm{~min}$ at RT while mixing. DMP was removed by cnetrifugation $\left(200 \mathrm{~g}, 1 \mathrm{~min}, 4^{\circ} \mathrm{C}\right)$. Column was washed twice with $500 \mu 11 \mathrm{M}$ Tris $\mathrm{pH} 7,4$. Beads were incubated with Tris for additional 2 hours at RT and stored at 4 ${ }^{\circ} \mathrm{C}$.

\subsubsection{Co-immunoprecipitation (Co-IP) with antibodies coated to PAS}

Purified mitochondria or cell suspensions were centrifuged at $10000 \mathrm{~g}$ for $5 \mathrm{~min}$. The amount of mitochondria was dependeing on the purpose of the IP and could range between $0,5 \mathrm{mg}$ and $5 \mathrm{mg}$ of mitochondria or cell lysates. The supernatant was removed and the pellet was resuspended in $200 \mu \mathrm{l}$ solubilization buffer (final: $50 \mathrm{mM}$ Tris/HCl, 50 mM NaCl, 10 \% Glycerol, 5 mM EGTA, $1 \%$ Digitonin, $200 \mu \mathrm{M}$ PMSF) by pipetting 20 times up and down. $600 \mu \mathrm{L}$ of solubilization buffer was added and again mixed by pipetting for 10 times. Lysates were incubated on ice for $30 \mathrm{~min}$. and centrifuged at $20000 \mathrm{~g}$ for $10 \mathrm{~min}$ at $4^{\circ} \mathrm{C}$. Unsolubilized debris was removed. Supernatant was taken and added to a prepared mobicol column (M2 Affinity Matrix (Sigma), 4G). To equilibrate the column, it was washed 3 times by adding washing buffer (final: $50 \mathrm{mM}$ Tris/ $\mathrm{HCl}, 50 \mathrm{mM} \mathrm{NaCl}, 10 \%$ Glycerol, $5 \mathrm{mM}$ EGTA, 0,3 \% Digitonin, $200 \mu \mathrm{M}$ PMSF) and centrifugation at $100 \mathrm{~g}, 30 \mathrm{sec}$. The lysate was incubated with the beads for 1 hour at $4{ }^{\circ} \mathrm{C}$ on a rotating wheel. Afterwards, unbound material was centrifuged at $200 \mathrm{~g}, 1 \mathrm{~min}$. Beads were washed 5 times with $500 \mu 1$ 
washing buffer (centrifugation steps $100 \mathrm{~g}, 30 \mathrm{sec}, 4{ }^{\circ} \mathrm{C}$ ). To elute proteins interacting with the coated antibody, glycin $(0,1 \mathrm{M}, \mathrm{pH} 2,8)$ was added to the column and incubated for $7 \mathrm{~min}$. at RT. Lysate was centrifuged for $1 \mathrm{~min}$. at $200 \mathrm{~g}$. Elution was mixed with $2 \mathrm{x}$ SDS loading dye (2.2.4.2), and subjected to further SDS-PAGE analysis. The unbound fraction of the Co-IP was subjected to 2D-BN-SDS-PAGE analysis (2.2.4.5) to study compositions of protein complexes.

\subsubsection{Specialized techniques}

\subsubsection{Radiolabeled in-vitro preprotein import into mitochondria}

Mitochondria from MEF cells were isolated via differential centrifugation. After the finalizing centrifugation step $(10.000 \mathrm{~g})$, mitochondria were resuspendend in Isolation buffer (Table 2.2) and adjusted to a relatively high-end concentration of around 20 $\mu \mathrm{g} / \mu \mathrm{l}$. Further pelleting and an additional resuspension step should have been prevented to avoid mechanical stress. Concentrated mitochondrial suspensions were aliquoted to single import time points. Simutaneously, preprotein lysate was prepared by in-vitro translation (2.2.3.8). One import reaction contained $30 \mu \mathrm{g}$ in $30 \mu \mathrm{l}$ mammalian Import reaction buffer (Table 2.2) and 1,5-3 $\mu 1$ preprotein lysate (2-10\%) were added for depicted time points at $30{ }^{\circ} \mathrm{C}$ and gentle shaking. $1 \mu \mathrm{AVO}$ mix (final: $8 \mu \mathrm{M}$ antimycin, $1 \mu \mathrm{M}$ valinomycin, $20 \mu \mathrm{M}$ oligomycin) was given to the negative control sample for $3 \mathrm{~min}$. to dissipate the membrane potential and to inhibit preprotein import. The same volume of Ethanol was added to the other samples. After 3, 7 and 15 min. respectively, mitochondrial import reactions were stopped by adding $1 \mu \mathrm{A}$ AVO mix and putting the samples back on ice. Samples were treated with Proteinase $\mathrm{K}(1 \mathrm{mg} / \mathrm{ml})$ for $10 \mathrm{~min}$. on ice and this digestion of non-imported precursors was inactivated with PMSF (200 mM in Ethanol) for $10 \mathrm{~min}$. on ice. Mitochondria were centrifuged at $18.000 \mathrm{~g}, 10 \mathrm{~min} ., 4{ }^{\circ} \mathrm{C}$. Supernatant was discarded, $150 \mu 1$ SEM buffer (Table 2.2) was added to wash out residual radioactivity and samples were pelleted again. Samples were further analysed by separation on SDSPAGE (2.2.4.2) to separate the imported mature form and non-imported preprotein under various time points to visualize the kinetics of in-vitro import reaction. As a second opportunity, mitochondria were solubilized by Blue-native solubilization 
buffer (Table 2.2) and applied to protein complex separation on BN-PAGE (2.2.4.4). Gels were proceeded for western blotting and analysis via digital autoradiography (2.2.4.7).

\subsubsection{Lipid extraction and Thin Layer Chromatography (TLC)}

Thin layer chromatography (TLC) is a chromatographic method, which was used to separate phospholipids. In the first place, lipids from purified mitochodnria were extracted. $1 \mathrm{mg}$ of purified mitochondria were resuspended with 1,5 $\mathrm{ml}$ Chloroformmethanol $(2: 1, \mathrm{v} / \mathrm{v})$ in a small glass test tube and shaken for 1 hour vigorously. While shaking the glass tubes resealed with parafilm at $4{ }^{\circ} \mathrm{C}$. Water $(300 \mu \mathrm{l})$ was added to the samples and vortexed for $1 \mathrm{~min}$. The samples were centrifuged at $1000 \mathrm{rpm}$ for 5 min. The glass tubes were fixed with a strip of filter paper in a $15 \mathrm{ml}$ falcon, so that they can be put into the centrifuge. The upper phase was the aqueous phase and was discarded after a centrifugation step. The lower phase was the solvent phase and was washed with a methanol/water mix $(1: 1, \mathrm{v} / \mathrm{v})$. The mix was vortexed and centrifuged as before. The solvent phase was transferred to a new glass tube. The samples were kept under a fume hood over night to dry the samples. The lipids were dissolved in $100 \mu \mathrm{l}$ of chloroform and subjected to further TLC analysis. The TLC glass plate was marked with pencil. From the bottom $2 \mathrm{~cm}$ space was left. Samples were applied to the TLC plate using glass capillaries and were spot several times on top of eachother. The TLC tank was filled with $100 \mathrm{ml}$ of Chloroform/Triethylamine/Ethanol/Water (30:35:35:7). 30 min prior to the run. The plate was put inside the TLC tank, which was filled with mobile phase solvent and the lid was closed. The run took about 3-4 hours to complete. In addtion to the samples some lipid standards were spotted beside.

Only one drop was sufficient. Common standards were cardiolipin, phosphatidylcholin and phosphatidylglycerol. After the TLC run the glass plate $i$ staken from the chamber and dried under a fume hood for several min. An oven was preheated at $180{ }^{\circ} \mathrm{C}$. The plate was submerged in the staining solution $\left(10 \% \mathrm{CuSO}_{4}\right.$ in $10 \%$ o-Phosphoric acid) and kept for 3-5 seconds. The plate was immediately incubated at $180{ }^{\circ} \mathrm{C}$ for $10 \mathrm{~min}$ until the spots on the plates appeared stong enough.

\subsubsection{ROS measurement by $\mathrm{H}_{2}$ DCFDA}


Reactive oxygen species (ROS) was detected by CM-H ${ }_{2}$ DCFDA (5-(and-6)chloromethyl-2',7'-dichlorodihydro fluorescein diacetate, acetyl ester). Isolated mitochondria from MEF cells were resuspended in assay buffer (Table 2.2). $100 \mu \mathrm{g}$ mitochondria were added to $0,3 \mathrm{ml}$ assay buffer containing $2 \mu 100 \mathrm{mM} \mathrm{CM}$ $\mathrm{H}_{2}$ DCFDA dye. Using a fluorescence spectrophotometer (Hitachi F-7000) changes in fluorescence was determined for 700 seconds (Excitation $498 \mathrm{~nm} /$ emission $525 \mathrm{~nm}$ ). ROS measurements were performed in triplicates.

\subsubsection{Measurements of mitochondrial calcium uptake}

Mitochondrial calcium uptake in MEF cells was measured in $1 \mathrm{mM}$ calcium (KrebsRinger) solution under the Cell Observer High Speed microscope fluorescence microscope (Zeiss). Mitochondrial calcium could interact with a calcium-binding site of the ratiometric sensor D3circular permuted Venus (D3cpv) causing that the donor fluorophor CFP come into Foerster radius with the acceptor YFP. D3cpv was a genetically encoded, mitochondrial matrix-targeted calcium sensor, which was transfected to MEF cells via electroporation (2.2.1.3). Only MEF cells with mitochondrial imported calcium sensor were measured. The signal of the donor fluorophor was substracted from the resulting FRET signal. The ratio of the FRET to donor (CFP) signal was calculated by the AxioVision software (Zeiss). Technically, transfected cells were seeded on coverslips and grown for 24 hours. Afterwards, the cover slip, which contained the adherent cells was put into a reservoir, where $1 \mathrm{ml}$ of Krebs Ringer solution was added. Then, basal mitochondrial calcium levels were monitored via the FRET/donor ratio under the microscope for $2 \mathrm{~min}$. $100 \mu \mathrm{M}$ ATP were added to stimulate calcium uptake into the cell and the development of stimulated mitochondrial calcium uptake was monitored for $5 \mathrm{~min}$. Measurements were performed in the lab of Ivan Bogeski.

\section{Results}




\subsection{Characterisation of MCU constituents in WT and TAZ KO MEF cells}

\subsubsection{Steady state levels of mitochondrial proteins}

Steady state levels of mitochondrial proteins were studied to obtain putative alterations in protein biosynthesis and turnover, mitochondrial protein import or posttranslational modifications. Alterations of protein steady state levels in BTHS models were already observed. Complex II levels are reduced in BTHS mouse hearts, whereas respiratory chain protein levels of complex I, III and IV are unaffected (Dudek et al., 2016). MEF cells were the main model system of this study and steady state levels of mitochondrial proteins in WT and TAZ KO MEF cells were addressed. Mitochondrial proteins of the respiratory chain complexes were detected after separation on SDS-PAGE and immunoblotting. Constituents of complex I (NDUFB8), complex II (SDHA, SDHB), complex III (RIESKE and complex V (ATP5B) did not show any altered protein levels in TAZ KO MEF mitochondria. However, the CIV components COX1, COX2 and COX4I-1 displayed reduced protein steady state levels in TAZ KO MEF mitochondria (Figure 3.1B). The protein levels of Mitochondrial Calcium Uniporter (MCU) constituents MCU, EMRE, MICU1, and MCUR1 were not affected in TAZ KO (Figure 3.1A). MCUb levels showed a slight reduction.

Concluding, protein steady state levels of MCU were not altered in TAZ KO MEF mitochondria. 


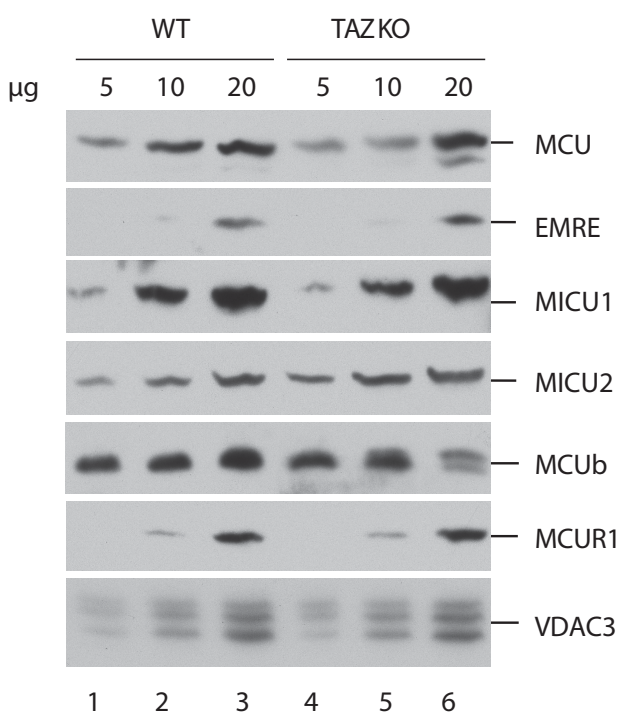


accessed and cleaved proteins exposed to the IMS, such as TIM23. MICU1 and MICU2 were degraded by PK in mitoplasts, but not in mitochondria, indicating that the MICU gatekeepers were localized at the IMS side of the IMM. Instead, TIM44 is localized to the mitochondrial matrix and protected against PK treatment. Sonication and PK treatment of mitoplasts degraded mitochondrial proteins of all compartments. Furthermore, the topology and the membrane-association of MICU gatekeepers was defined in WT and TAZ KO MEF mitochondria. MICU1 and MICU2 contain a Nterminal MTS, which is cleaved after protein import (Petrungaro et al., 2015), but it was still unclear, whether MICU1 and MICU2 are integral membrane proteins or just inner membrane-associated in the IMS. Carbonate and Triton X-100 extractions of membrane-bound mitochondrial proteins provide information, if a mitochondrial protein contains a transmembrane span (Figure 3.2B). Carbonate solution of $\mathrm{pH} \mathrm{10,8}$ extracted the membrane-associated protein TACO1 from the pellet fraction partly and could extract TACO1 completely into the soluble fractions upon $\mathrm{pH} 11,5$. The integral membrane protein TOM20 was extracted from membranes only by Triton X100. The majority of MICU2 was extracted into the soluble fraction by carbonate treatment in both carbonate conditions, indicating that MICU2 is not an integral protein, but membrane-associated. MICU1 and MICU2 contain cardiolipin-binding domains (Kamer et al., 2017) and cardiolipin remodeling might be essential for membrane association. Thus, the carbonate extraction pattern of MICU2 in WT mitochondria was compared with its pattern in TAZ KO. The hypothesis was, that less membrane association could be displayed by a higher share of soluble MICU2, but MICU2 extraction pattern did not show differences. MICU2 association to the inner membrane was not taffazin-dependent. In fact, TACO1 was more extracted from membranes in TAZ KO.

Concluding, the MCU gatekeepers MICU1 and MICU2 are associated to the inner mitochondrial membrane and exposed to the innermembrane space. MICU2 is not a transmembrane span-containing mitochondrial protein and MICU2 association to the innermitochondrial membrane is not affected in TAZ KO MEF mitochondria. 
A

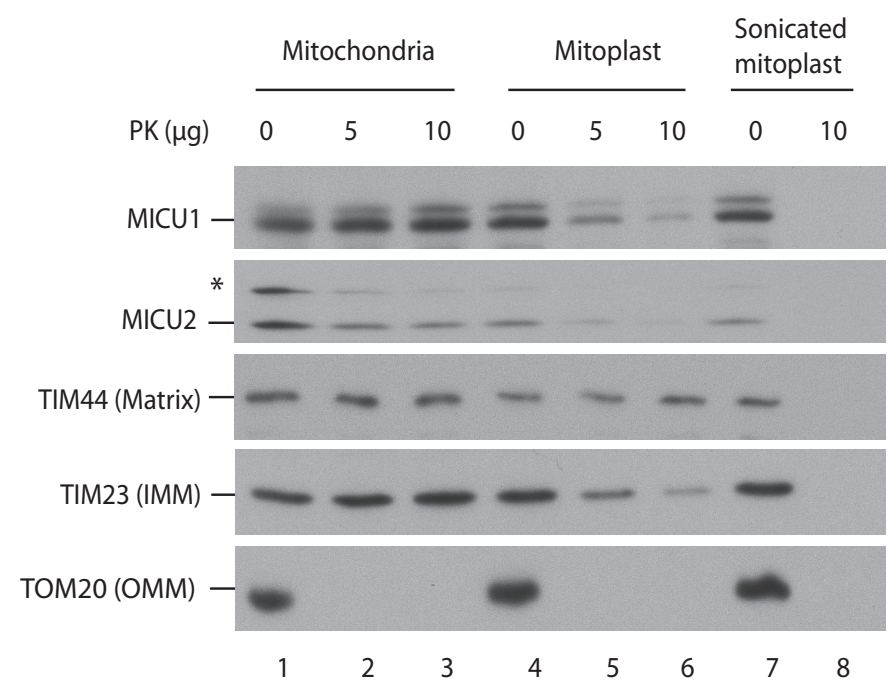

B

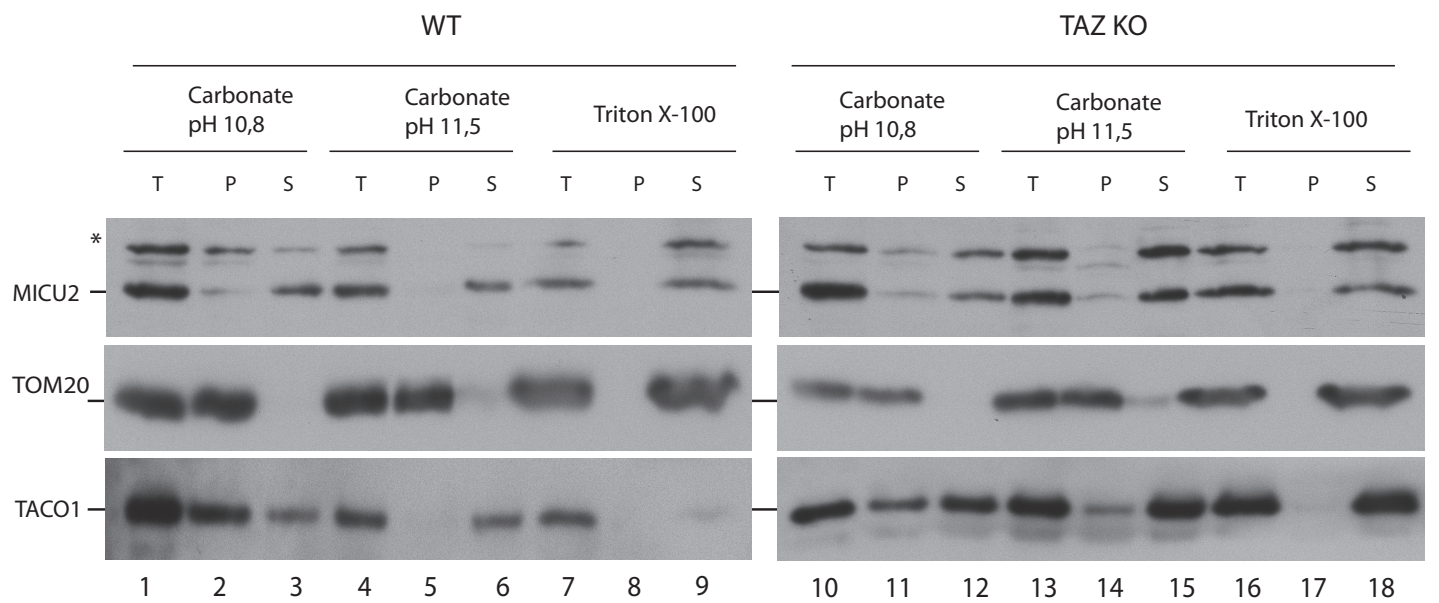

Figure 3.2: Micu1 and Micu2 are associated to the inner mitochondrial membrane and exposed to the innermembrane space

(A) Mitochondria from WT MEF cells were resuspended in isotonic buffer to maintain mitochondrial outer membrane. Mitochondria were swelled in hypotonic buffer for 15 minutes to gain mitoplasts. Samples were separated on a SDS-PAGE resolving gel (12,5\%). Indicated proteins were detected by immunoblotting. (IMM, Inner mitochondrial membrane; OMM, Outer mitochondrial membrane, PK, Proteinase $\mathrm{K}$, * unspecific band

(B) Mitochondria from WT and TAZ KO MEF cells were resuspended in freshly prepared carbonate (pH 10,8 and $\mathrm{pH} 11,5$, respectively) and Triton X-100 solutions. Samples were pelleted at $100.000 \mathrm{~g}$ for 45 minutes. Pellets and supernatants were divided and separated on a SDS-PAGE resolving gel $(12,5 \%)$. Indicated proteins were detected by immunoblotting. T, Total, P, Pellet, S, Soluble fraction,

* unspecific band

\subsection{Analysis of MCU complex composition and organization in TAZ KO MEF} cells 


\subsubsection{D-BN-SDS-PAGE analysis of MCU/EMRE-containing complexes in MEF cells}

MCU complexes in the MegaDalton (MDa) range of mouse brains and HEK cells undergo reorganization processes depending on the turnover and expression of EMRE (König et al., 2016). To address, if MCU complexes display altered organization or composition in a different lipid environment, mitochondria were purified, solubilized in digitonin, and protein complexes separated by BN-PAGE analysis. MCU complexes were detected by immunoblotting, and two separate MCU complexes with different molecular weights were detected (Figure 3.3A). WT mitochondria contained a $1000 \mathrm{kDa}$ (named MCU-2) and a $500 \mathrm{kDa}$ MCU complex (named MCU-3), where MCU was more abundant in the higher molecular weight form. MCU complexes in TAZ KO mitochondria showed a ratio shift. MCU was more distributed to the lower complex in TAZ KO. Both MCU complexes in TAZ KO were slightly shifted potentially caused by the different lipid composition of the micelles in BN-PAGE analyses. The interaction partner EMRE could not be detected in the first BN-PAGE dimension. The separated mitochondrial fractions were again separated on a SDSPAGE in the second dimension (Figure 3.3B). A third MCU complex in the higher MDa range was apparent (named MCU-1 complex), which was not present in TAZ $\mathrm{KO}$ mitochondria. These analyses showed the MCU complex ratio shift between both phenotypes. EMRE signals comigrated with MCU signals. The MCU antibody detected an additional signal in the MDa range, which was not comigrating with EMRE signals.

Concluding, EMRE-containing MCU complexes display altered complex organization in TAZ KO MEF mitochondria. MCU protein is shifted to lower molecular weight forms of MCU in TAZ KO MEF cells. 


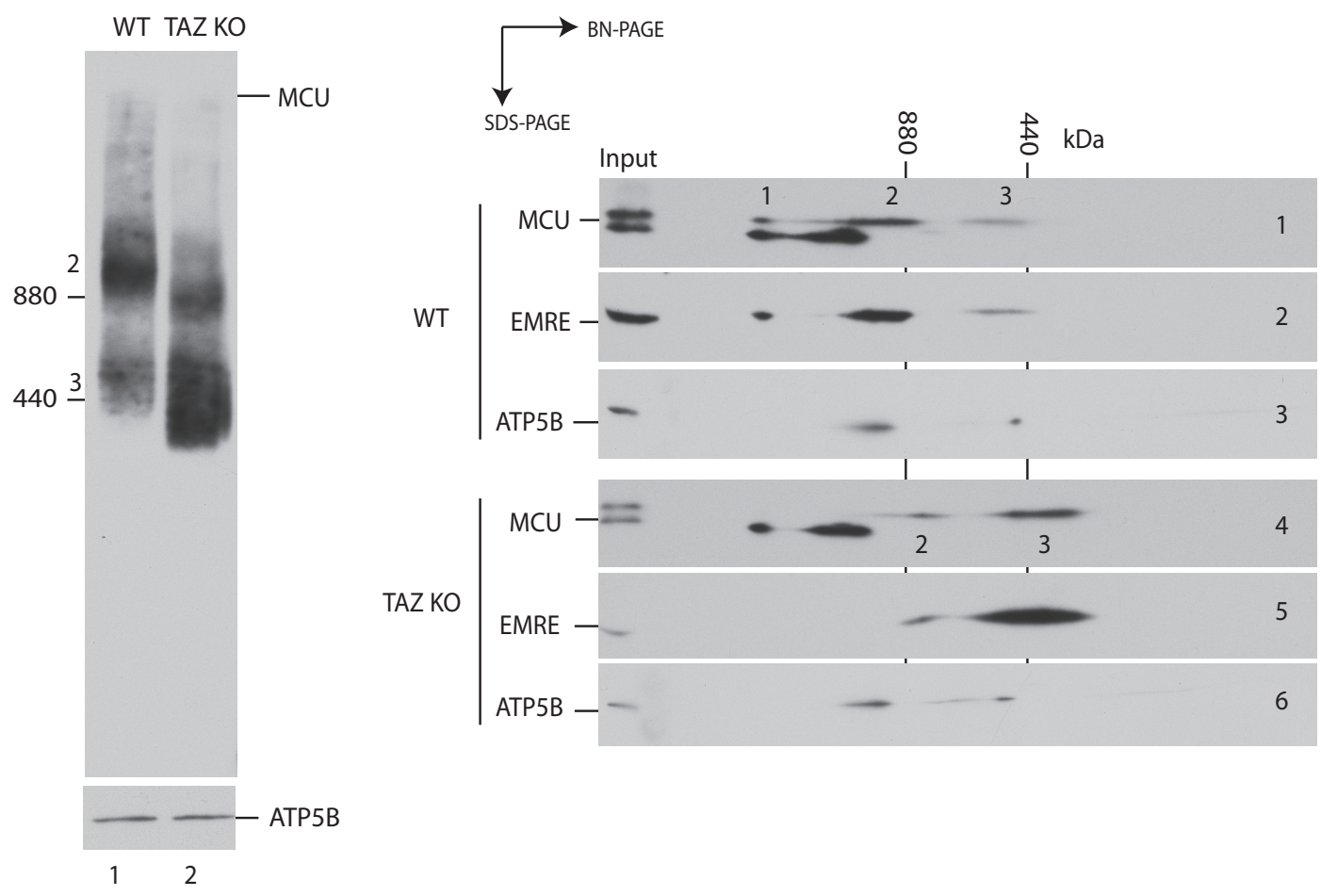

Figure 3.3: EMRE-containing MCU complexes undergo a ratio shift in TAZ KO MEF mitochondria

(A) Mitochondria from WT and TAZ KO MEF cells were solubilized by $1 \%$ digitonin and separated by a gradient BN-PAGE resolving gel (4-13\%). Indicated proteins were detected by immunoblotting.

(B) Mitochondria from WT and TAZ KO MEF cells were solubilized and separated as described in (A). Single BN-PAGE lanes were cutted out and separated on SDS-PAGE in the second dimension (2D-BN-SDS-PAGE analysis). Protein complexes were detected by western blotting against MCU, EMRE and ATP5B

\subsubsection{EMRE-containing complexes interact with MCU oligomers}

To get more information about the composition of MCU complexes in the MDa range, an EMRE depletion coimmunprecipitation (Co-IP) analysis was performed. EMRE and its interaction partner MCU were pulled down by an EMRE antibody (Figure 3.4A). The two protein bands detected by the MCU antibody displayed different interaction pattern with EMRE. The upper band was pulled down by the EMRE antibody and the lower band was unaffected. The unbound fractions of the CoIP were analysed by 2D-BN-SDS-PAGE analyses (Figure 3.4B). The two MDa MCU complexes (named MCU-1 and MCU-2, Figure 3.4B lane 1) were exposed in the control fractions, which comigrated with EMRE. Those complexes disappeared after 
pulldown of EMRE. Thus, EMRE-containing complexes were not just comigrating with MCU complexes, those complexes were EMRE-containing MCU complexes. However, the unknown signal in the MDa range remained stable (Figure 3.4B, lane 1 and 4) and it was not coimmunoprecipitated with EMRE and MCU. Therefore, the unknown signal in the MDa range was not an EMRE-containing protein complex.

A
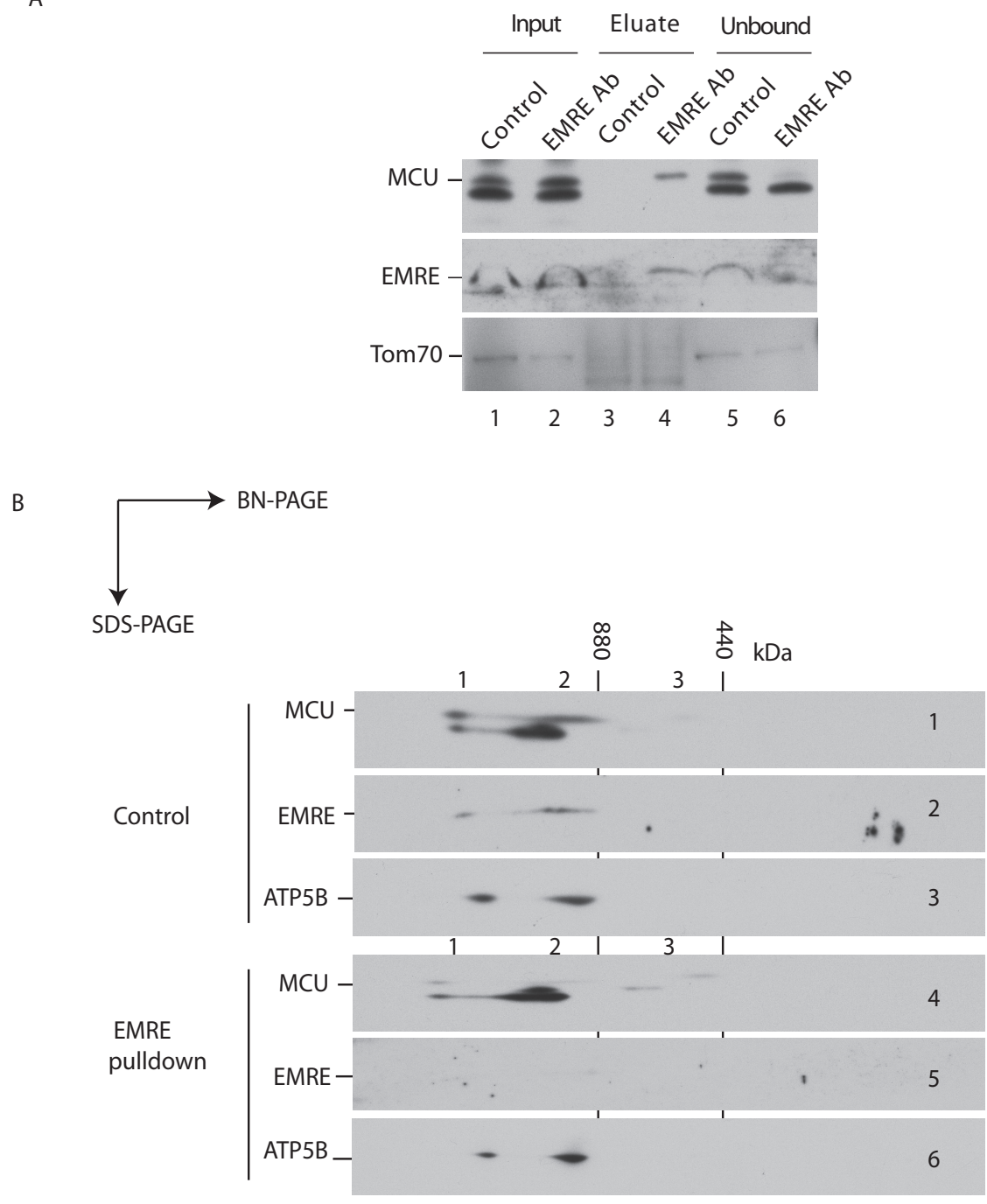

Figure 3.4: EMRE-containing complexes interact with MCU oligomers

(A) Mitochondria from WT MEF cells were coimmunprecipitated by EMRE and Tafazzin antibody in $1 \%$ digitonin and EGTA-containing solubilization buffer. Samples were separated on 10-18 \% gradient SDS-PAGE and immunoblotted against EMRE and MCU

(B) Native unbound fractions from (A) were separated by 2D-SDS-BN-PAGE analysis and immunoblotted against EMRE, MCU and ATP5B.

\subsubsection{Verification of MCU signal specificity by MCU RNA silencing}


The second approach to charaterize the double band detected by the MCU antibody after immunoblotting was a knockdown of MCU mRNA and protein levels. MCU RNA silencing by siRNA could address the specificity of the MCU antibody. After 3 days of MCU RNA knockdown MEF cell lysates (Figure 3.5A) and mitochondria (Figure 3.5B) were harvested. The comparison of MCU from WT and non-target siRNA (NT) transfection showed that the treatment itself did not affect MCU levels (Figure 3.5A). Two siRNAs against MCU mRNA were tested and it was proceeded with siRNA number 2 . The knockdown of MCU after mitochondria isolation revealed that EMRE and Micu1 levels were reduced as well (Figure 3.5B). The upper band of the MCU antibody double band was strongly reduced and the lower band remained unaffected (Figure 3.5A+B). These findings clarified that the lower band was not MCU.

A

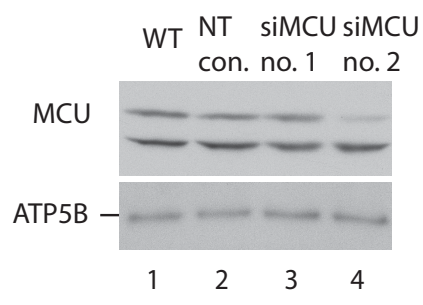

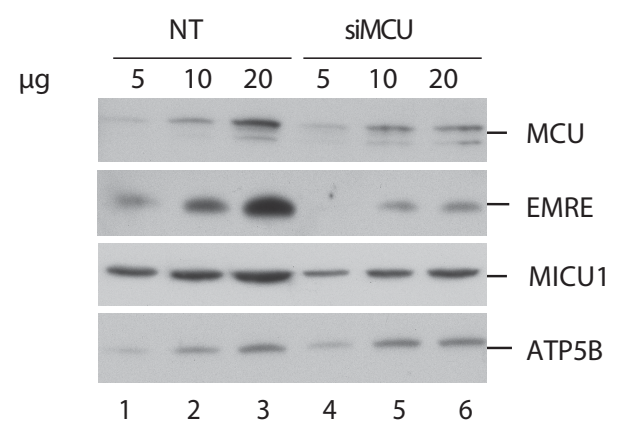

Figure 3.5: MCU antibody (sigma) reveals a double band and the upper one is specific (A) Non-target control siRNA (NT con.) and siRNA against MCU mRNA were transfected to WT MEF cells $(50 \mathrm{ng} / \mu \mathrm{L})$. After 3 days cells were harvested and lysed by a denaturing buffer. Samples were separated on SDS-PAGE $(12,5 \%)$ and immunoblotted against MCU and ATP5B

(B) After MCU mRNA silencing as described in (A), mitochondria was isolated by differential centrifugation. Samples were separated on a 10-18\% gradient SDS-PAGE and immunoblotted against EMRE, MCU, Micu1 and ATP5B

\subsubsection{The interaction of MCU and EMRE is not affected in TAZ KO MEF mitochondria}

The MCU complexes shifted between higher and lower molecular weight forms depending on tafazzin activity as shown before (Figure 3.3). The MCU complexes have always been interacting with EMRE. The interaction of MCU and EMRE was addressed. Therefore, FLAG-tagged human MCU was overexpressed in WT MEF cells and endogenous EMRE was coimmunprecipitated by FLAG beads (Figure 
3.6A). The degree of EMRE and MCU-FLAG interaction was unaffected in TAZ KO MEF cells. The sequence homology of murin and human mature MCU is $100 \%$, so that the different origins of MCU-FLAG (human) and EMRE (mouse) did not affect the interaction. This was confirmed by coimmunprecipitation of MCU-FLAG and EMRE and visualization of EMRE-containing MCU complexes after 2D-BN-SDSPAGE analysis (Figure 3.6B). The native eluate of the MCU-FLAG-IP from WT cells was loaded and separated on BN-PAGE. MCU-FLAG was organized in the two MDa MCU complexes (complexes MCU-1 and MCU-2) recapitulating earlier experiments (Figures 3.3 and 3.4). Immunoblotting against endogenous MCU complexes showed that MCU-FLAG contained endogenous MCU as well. Indicating, that MCU-FLAG and endogenous MCU formed heterogenous MCU pores which were interacting with EMRE. The finding of unaltered MCU-EMRE interaction in TAZ KO MEF cells was further confirmed by coimmunprecipitation of EMRE as a different bait (Figure 3.6C). Other MCU interaction partners known from the literature, such as $\mathrm{MCUb}$, MCUR1 and the gatekeepers MICU1 and MICU2 could not be detected by diverse approaches (specific depletion IPs, RNAi, antibody shift assays or variation of antibodies for IP or immunoblotting).

The Co-IP studies of MCU and EMRE displayed, that the interaction of MCU and EMRE was not affected in TAZ KO MEF mitochondria. 
A

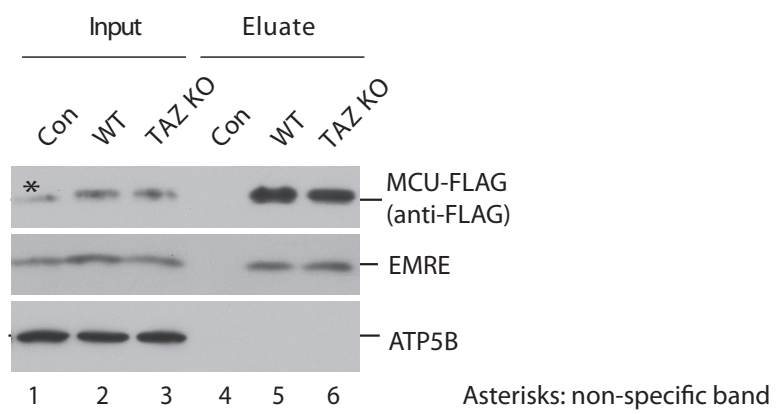

B

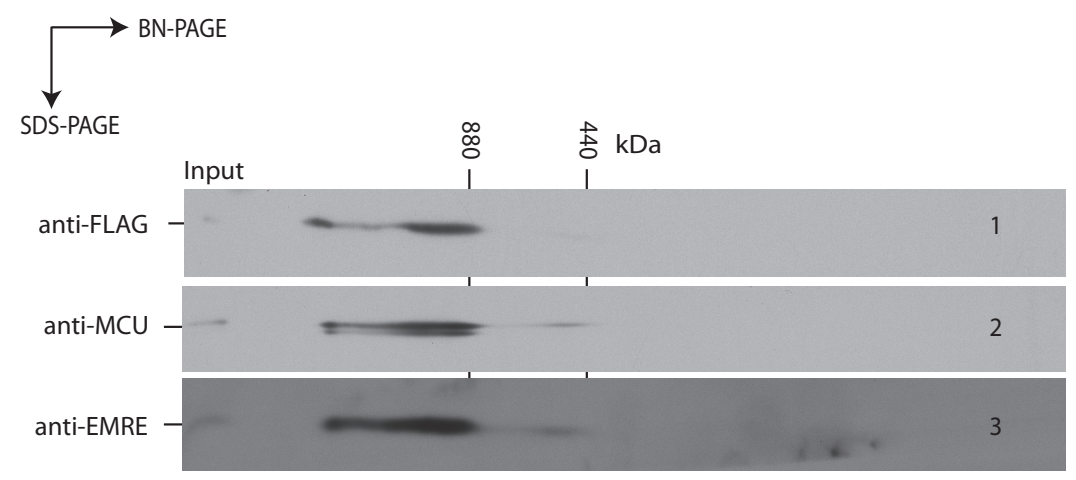

$\mathrm{C}$

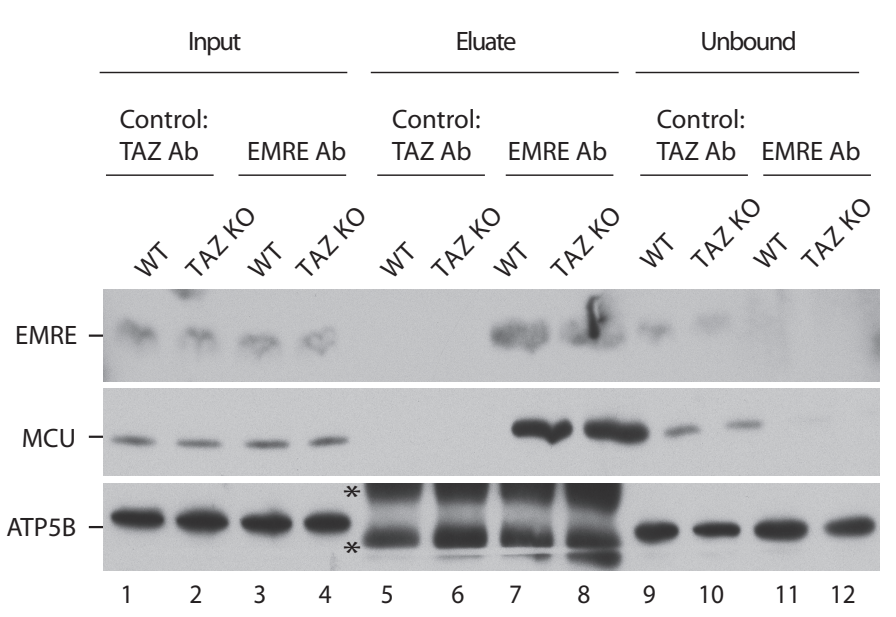

Asterisks: IgG chains

Figure 3.6: Interaction of MCU and EMRE is unaffected in TAZ KO MEF cells

(A) WT and TAZ KO MEF cells were transiently transfected with human MCU-FLAG construct. After 3 days, FLAG-immunoprecipitation was performed and samples separated on 12,5\% SDS-PAGE

(B) MCU-FLAG was overexpressed in WT MEF cells. Native eluate fractions from FLAGimmunoprecipitation (A) were separated on BN-PAGE (4-13\% resolving gel). Single BN gel stripes were further separated on SDS-PAGE (16,5\% resolving gel) and immunoblotted against FLAG, EMRE and MCU.

(C) Mitochondria from WT and TAZ KO cells were coimmunprecipitated by EMRE and tafazzin antibodies conjugated to protein A sepharose. Samples probed on SDS-PAGE were immunoblotted against EMRE, MCU and ATP5B

\subsubsection{MICU1 pulldown via depletion IP impact MCU MDa complex}


The EMRE depletion Co-IP in section 3.2.2 illustrated that MEF MCU complexes contained EMRE. To address the further composition of MCU, two hypotheses were considered. First, MCU complexes in the MDa range (MCU-1, MCU-2) could dissociate in MEF TAZ KO mitochondria, causing the lack of subunits in MDa MCU complexes. The MICU gatekeepers were considered to interact with MCU tendentially in one of the MDa MCU complexes and not in the $\mathrm{kDa}$ MCU complexes. The other possibility was, that the $1000 \mathrm{kDa}(\mathrm{MCU}-2)$ and the $500 \mathrm{kDa} \mathrm{MCU}$ (MCU3) complexes are just oligomers. Size-wise it was indicative that both complexes are MCU monomers and dimers. To test which of the both hypothesis was true, a MICU1 depletion Co-IP was performed. The unbound fractions were loaded and separated on 2D-BN-SDS-PAGE (Figure 3.7). The MICU1 antibody pulled down the higher MDa MCU complex (MCU-1) and the $1000 \mathrm{kDa}$ complex (MCU-2) remained unaffected. This was an indication, that the MICU gatekeepers reside in the higher MDa form (MCU-1) and not in the kDa complexes (MCU-2 and MCU-3). The higher MDa complex (MCU-1) was not detectable in TAZ KO (Figure 3.3) indicating that MCU complexes in TAZ KO cells did not interact with MICU1.

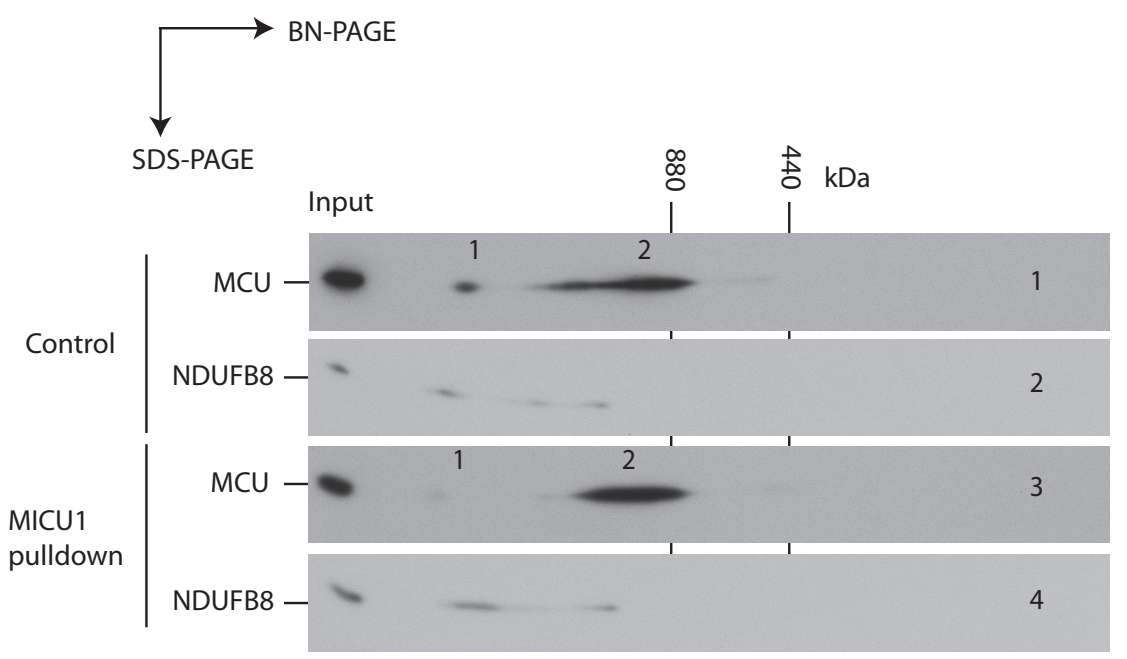

Figure 3.7: The MDa MCU complex contains the gatekeeper MICU1

WT MEF cell lysates ( $1 \mathrm{mg}$ ) were solubilized by a $0.5 \%$ digitonin- and an EGTA-containing buffer. Micul antibody (Cell signaling) and the control antibody (raised against tafazzin, homemade) were coated to protein A sepharose. Micul and putative interaction partners were coimmunprecipitated from WT cell lysates. Unbound fractions of the coimmunprecipitation were separated on a 4-13\% BN-PAGE resolving gel. Single BN gel stripes were further separated on SDS-PAGE (16,5\% resolving gel) and immunoblotted against MCU and NDUFB8. 


\subsubsection{Complementation of TAZ KO MEF cells by exogenous tafazzin expression: Isolation of Taz-FLAG Isoform 2 and functional analysis via TLC}

It was necessary to clarify, if the MCU complex reorganization in TAZ KO cells was caused by the lack of tafazzin activity or by other unspecific origins. Therefore, 2 different mouse TAZ-FLAG isoforms were cloned by Arpita Chowdhury and transiently transfected to MEF cells via electroporation. Both TAZ-FLAG isoforms were weakly expressed in MEF cell lysates and were not detectable via western blotting (Figure 3.8A). Thus, the FLAG-tagged tafazzin isoforms were immunoprecipitated and expression was confirmed. Isoform 2 was sufficiently expressed and further experiments were performed with this isoform.

The expression of exogenous TAZ-FLAG in TAZ KO MEF cells was characterized on the functional level. Thin-layer-chromatography (TLC) showed, if the genetic modification of TAZ KO cells affected the lipid composition (Figure 3.8B). The ratio of cardiolipin (CL) to its precursor monolysocardiolipin (MLCL) might be reduced TAZ KO MEF cells. We wanted to check whether genetic complementation of tafazzin in TAZ KO cells could shift the CL/MLCL ratio to the WT lipid composition or not. WT MEF cells revealed cardiolipin, but no monolysocardiolipin. The precursor MLCL appeared in TAZ KO MEF cells and disappeared in TAZ-FLAG expressing TAZ KO cells. Levels of other phospholipid, such as PG and PC were not affected in this approach.

Transient transfection and expression of Tafazzin-FLAG in TAZ KO MEF cells were established and rescued the CL/MLCL ratio in TAZ KO MEF cells. 


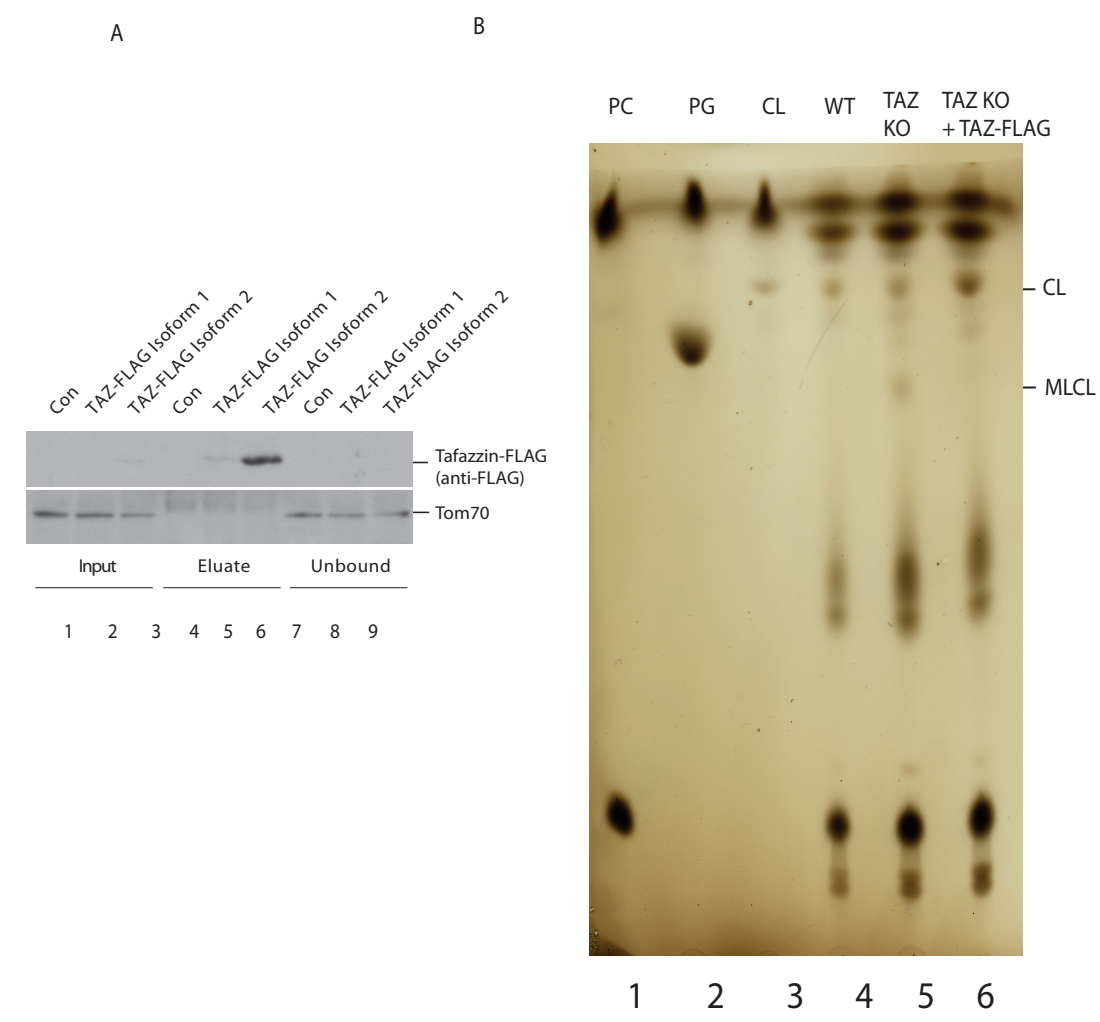

Figure 3.8: Altered lipid composition of TAZ KO MEF cells can be rescued by complementation with tafazzin.

(A) Mouse tafazzin isoforms were transfected to TAZ KO MEF cells by electroporation. Native immunoprecipitation of FLAG-tagged taffazin was performed with $1 \mathrm{mg}$ of total cell lysates.

(B) Lipids were extracted from WT, TAZ KO and TAZ-FLAG containing TAZ KO MEF mitochondria by thin-layer chromatography (TLC). Lipid migration was controlled by parallel separation of standard lipid solutions (PC, phosphatidylcholine; CL, cardiolipin; MLCL, monolysocardiolipin; PG, phosphatidylglycerol)

\subsubsection{MCU complex dissociation and respiratory chain remodeling in TAZ KO MEF mitochondria are rescued by tafazzin complementation}

To address, if tafazzin complementation in TAZ KO MEF cells rescues the MCU complex organization, MCU complexes from TAZ-FLAG expressing TAZ KO MEF cells were separated on 2D-BN-SDS-PAGE. In fact, tafazzin complementation in TAZ KO MEF cells rescued the altered MCU organization in TAZ KO and returned it to the WT MCU complex distribution. This finding highlighted, that the $500 \mathrm{kDa}$ MCU complex (MCU-3) did not require appropriate remodeling of cardiolipin for its structural integrity, whereas the stability of the $1000 \mathrm{kDa}$ MCU complex was caused by appropriate tafazzin expression and function (Figure 3.9A).

Respiratory chain complexes are remodeled in TAZ KO MEF cells and respirasomes were drastically reduced (Chowdhury et al., 2018). Expression of exogenous TAZ- 
FLAG in TAZ KO MEF cells could confirm the specific dependency of tafazzin on respiratory chain complex stability (Figure 3.9B). Respirasomes occured after tafazzin complementation in TAZ KO and the migration pattern of the single OXPHOS oligomers were comparable to WT. The different lipid/cardiolipin content in the micelles of solubilized mitochondria could alter the migration behavior on BN-PAGE. The data indicate, that MCU complex dissociation and respiratory chain remodeling in TAZ KO MEF mitochondria were rescued by tafazzin complementation.

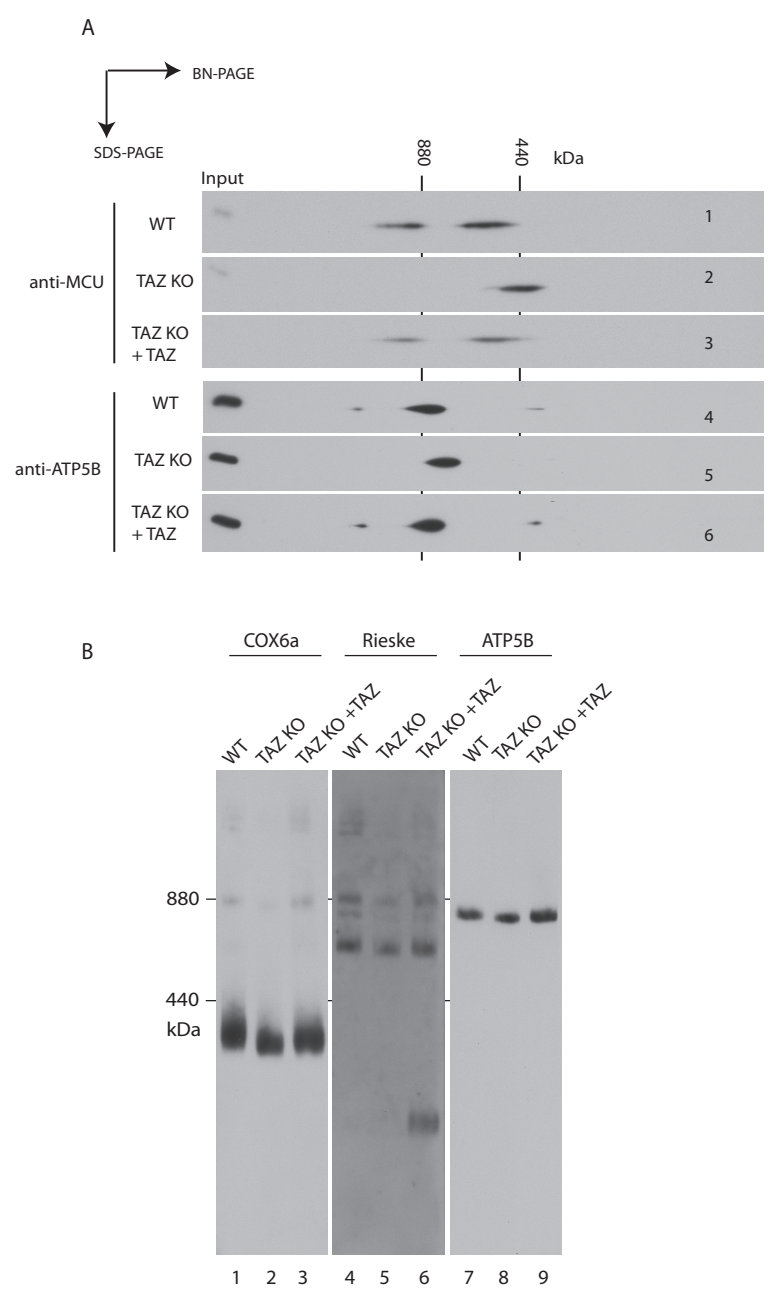

Figure 3.9: MCU complex organization and respiratory chain remodeling is tafazzin-dependent. (A) Lysates from WT, TAZ KO, and tafazzin-complemented TAZ KO MEF cells were solubilized in $1 \%$ digitonin and separated in a $4-13 \%$ BN-PAGE resolving gel. Single BN gel stripes were further separated on SDS-PAGE (16,5\% resolving gel) and immunoblotted against MCU and ATP5B.

(B) Lysates from WT, TAZ KO, and tafazzin-complemented TAZ KO MEF cells were solubilized in $1 \%$ digitonin and separated on a 4-13\% BN-PAGE resolving gel system. Respiratory chain complexes were detected by immunoblotting against Cox6a, Rieske and ATP5B 


\subsubsection{MCU complex stability in MEF cells is dependent on appropriate cardiolipin biosynthesis, but not reactive oxygen species (ROS)}

The structural integrity of MCU depends on cardiolipin remodeling (3.2.7). We asked whether MCU complex organization is dependent on taffazin Furthermore, we monitored, if impaired cardiolipin biosynthesis affects MCU complex stability. Therefore, the cardiolipin synthase (CRLS1 gen) was silenced by an RNAi approach for 3 days (Figure 3.10A) and MCU complexes were separated from cell lysates. Non-targeted control MEF cells revealed a $500 \mathrm{kDa}$ and a $1000 \mathrm{kDa}$ MCU complex. SiCRLS1-treated MEF cells just contained the $500 \mathrm{kDa}$ MCU complex (Figure 3.10B). As a consequence, higher MCU oligomers, at least in the MDa range, require appropriate cardiolipin levels to maintain structural integrity.

MCU activity and complex oligomerization were discussed to be regulated by the mitochondrial redox status via S-glutathionylation at the N-terminal domain (NTD) (Dong et al., 2017). In addition, MICU1 and MICU2 dimerization might be dependent on the redox status in the IMS (Petrungaro et al., 2015). To study the impact of reactive oxygen species (ROS) on protein complex organization mitochondrial ROS production was quenched by mitotempo for 24 hours and samples analyzed on 2DBN-SDS-PAGE (Figure 3.10C). The success of the treatment was confirmed by fluorescence-based ROS measurements via 2', 7'-dichlorofluorescin (DCFDA). ROS production in TAZ KO cells was significantly increased and were rescued by addition of mitotempo (Figure 3.10D). The phenotype of increased ROS levels in TAZ KO MEF cells went along with Chowdhury et al., 2018. Hence, reduction of ROS does not alter MCU complex integrity in both, WT and TAZ KO MEF cells. 

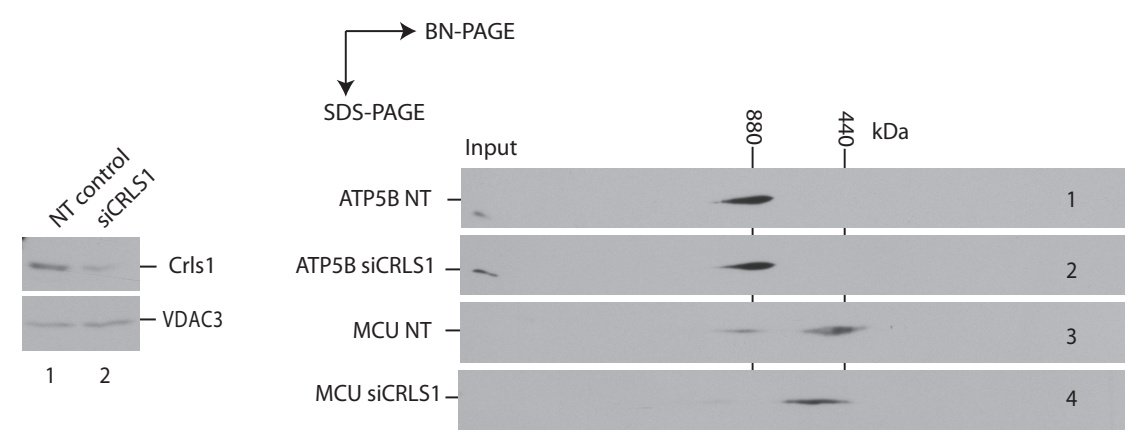

C

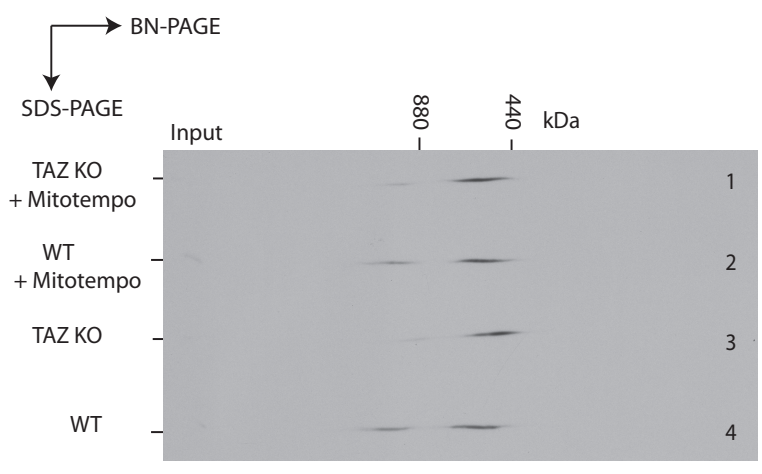

D

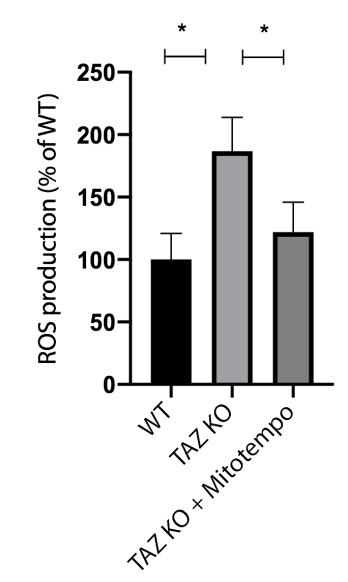

Figure 3.10: MCU complex organization depends on cardiolipin biosynthesis

(A) Cardiolipin synthase mRNA in MEF cells was silenced by siRNA for three days. After harvesting, cells were lysed and separated on 12,5\% SDS-PAGE and immunoblotted against VDAC3 and cardiolipin synthase.

(B) MEF cells from (A) were solubilized and resolved by 2D-BN-SDS-PAGE analysis. Protein complexes were detected by immunoblotting against MCU and ATP5B.

(C). MEF cells were treated with the ROS scanvenger Mitotempo (50 $\mu \mathrm{M}$ final concentration) for 24 hours. Cells were solubilized by digitonin and separated by 2D-BN-SDS-PAGE analysis. MCU complexes were detected by western blotting.

(D) Function of ROS scavenger Mitotempo was examined with isolated mitochondria by fluorimetric ROS measurements via DCFDA $\left(2^{\prime}, 7^{\prime}\right.$-dichlorofluorescin $)(n=3$, mean \pm SEM)

\subsubsection{Quantification of MCU complex ratio shift in WT and TAZ KO MEF cells}

The ratio shifts of MCU complexes in WT and TAZ KO MEF cells were quantified to address, if this phenotype is statistically relevant. The ratio shift of the $500 \mathrm{kDa}$ and $1000 \mathrm{kDa}$ MCU complexes in WT and TAZ KO MEF cells were quantified by densiometric approach via ImageJ (Figure 3.11A). MCU complexes in WT showed a ratio shift from the higher to the lower molecular weight form of around 60-to-40 percent. MCU was more distributed to the $500 \mathrm{kDa}$ form in TAZ KO and the ratio 
shift from the higher to the lower molecular weight form was approximately 10-to-90 percent. This ratio shift appeared differently after MCU separation from cell lysate (Figure 3.11C) or isolated mitochondria (Figure 3.11B). The MCU complex ratio in both genotypes was more shifted to the lower molecular weight form after cell lysate separation in comparison to isolation from mitochondria and less in the higher oligomer in tendency.

However, independently from the procedure of purifying mitochondria, the MCU complex ratio shifts in WT and TAZ KO MEF cells are statistically relevant.

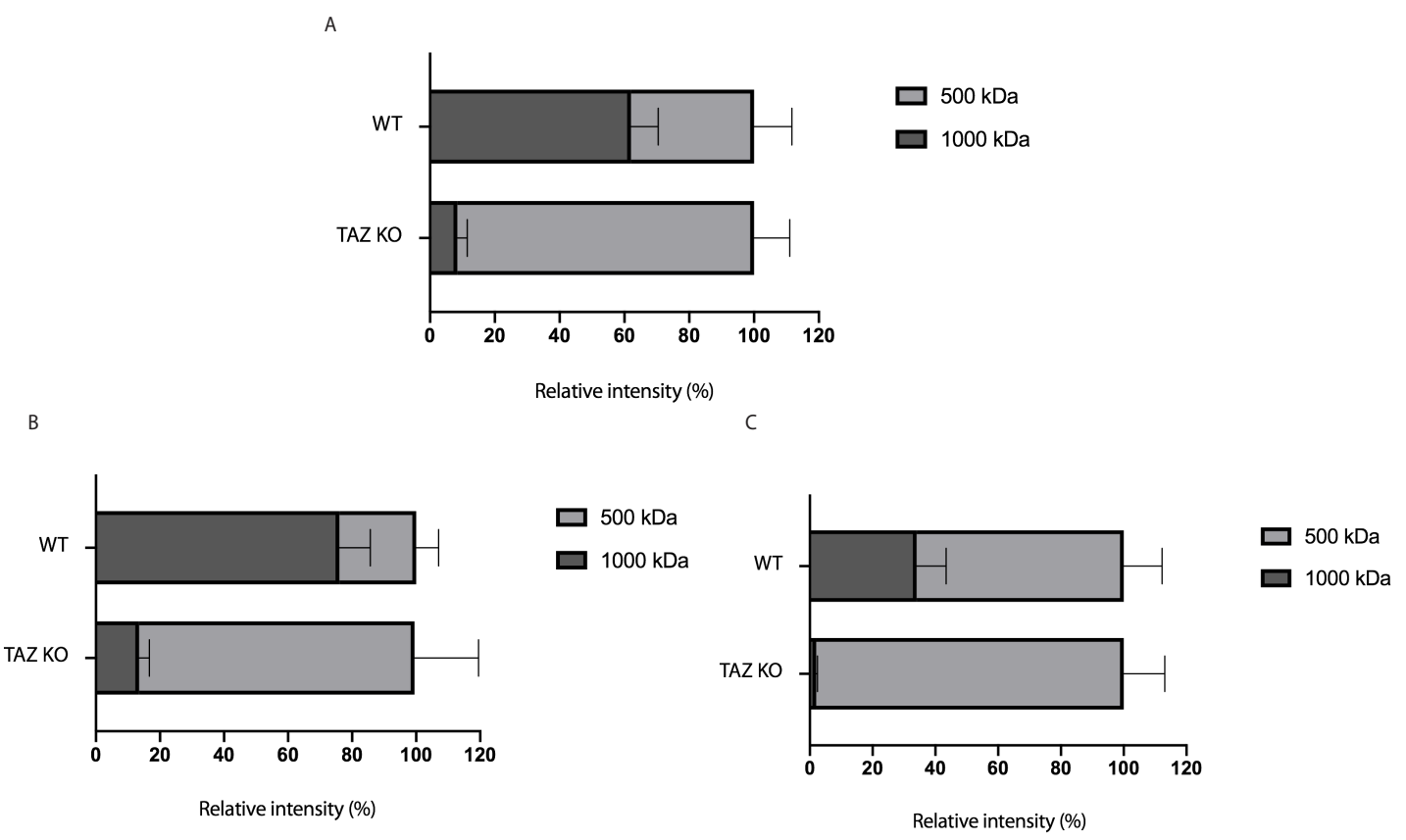

Figure 3.11: Higher-order MCU complexes shift to lower molecular weight forms in TAZ KO MEF cells

(A, B, C) MCU complexes were quantified by densiometric analysis (ImageJ). The signal intensity of the $1000 \mathrm{kDa}$ and the $500 \mathrm{kDa}$ MCU complexes were set to $100 \%$ per genotype and the shares of both complexes per genotype were compared $(n=8$, mean \pm SEM).

(A) Quantifications of MCU complex ratios in WT and TAZ KO MEF cells. Either whole cells were harvested and directly solubilized for 2D-BN-SDS-PAGE analysis or mitochondria from MEF cells were used $(n=8$, mean \pm SEM).

(B) Quantifications of MCU complex ratios in WT and TAZ KO MEF mitochondria ( $\mathrm{n}=5$, mean \pm SEM).

(C) Quantifications of MCU complex ratios in WT and TAZ KO MEF cell lysates $(\mathrm{n}=3$, mean \pm SEM).

\subsection{Mitochondrial calcium uptake is affected in TAZ KO cells}

The reorganized MCU complexes in TAZ KO MEF cells could affect mitochondrial calcium uptake. To measure mitochondrial calcium uptake, whole cells were 
transiently transfected with a mitochondrial matrix-targeted calcium sensor, called D3 circularly permuted Venus (D3cpv) (Wallace et al., 2008). MEF cells were measured in $1 \mathrm{mM}$ calcium (Krebs-Ringer) solution under a fluorescence microscope. Mitochondrial calcium could interact with a calcium-binding site of the sensor causing the donor fluorophor CFP come into Förster radius with the acceptor YFP. The signal of the donor fluorophor was substracted from the resulting FRET signal. Only MEF cells with mitochondrial imported calcium sensor were measured. This ratiometric calculation led to the finding, that the basal calcium uptake into TAZ KO MEF mitochondria was increased (Figure 3.12). This observation would fit to lack of MICU1 in MCU complexes in TAZ KO MEF mitochondria. MCU pores are constitutive active when gatekeepers are not interacting (Koenig et al., 2016; Tsai et al., 2017). The observation from figure 3.3 and 3.4 indicated, that MICU gatekeepers were reduced on the MCU pore in TAZ KO. However, the role of MCUR1 and MCUb and other unknown MCU interaction partners could not be determined in this study. The composition of the $1000 \mathrm{kDa}$ and $500 \mathrm{kDa}$ MCU complexes is still unsolved (beside MCU and EMRE) and a conclusive model of lacking gatekeepers, which indicate higher basal calcium uptake was too early at this stage of knowledge. The measurements of mitochondrial calcium uptake were performed by Zurine Bonilla del Rio in the lab of Ivan Bogeski.

Concluding, basal mitochondrial calcium uptake is increased in TAZ KO MEF mitochondria.

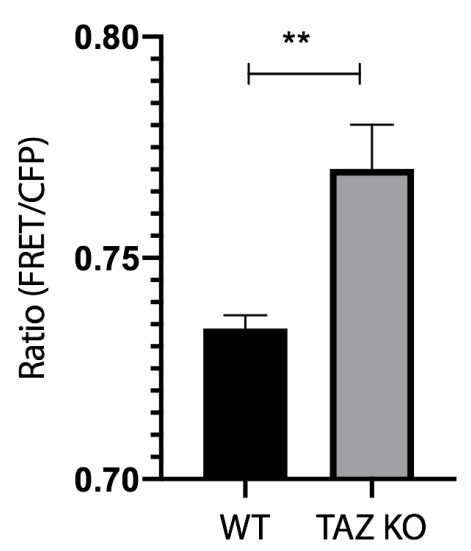

Figure 3.12 Basal mitochondrial calcium uptake is increased in TAZ KO MEF cells Mitochondrial calcium uptake of cultured WT and TAZ KO MEF cells was detected by the genetically encoded, mitochondrial matrix-targeted calcium sensor D3cpv. Cells were measured in $1 \mathrm{mM}$ KrebsRinger solution at Cell Observer High Speed microscope (Zeiss). The ratio of FRET to donor (CFP) signal was calculated by AxioVision software (Zeiss) $(\mathrm{n}=3$, mean $\pm \mathrm{SEM}$, ANOVA one-way). The 
measurements of mitochondrial calcium uptake were performed by Zurine Bonilla del Rio in the lab of Ivan Bogeski.

\subsection{Import and assembly of MCU and EMRE in WT/TAZ KO MEF mitochondria}

\subsubsection{Tafazzin deficiency does not affect translocases in MEF cells}

The altered MCU complex organization in TAZ KO MEF cells gave rise to the question, if MCU complex assembly of newly synthetized and imported MCU components is affected in TAZ KO mitochondria. In the first place, the protein steady state levels of core constituents of mitochondrial translocases TIM23, TIM22 and TOM were examined (Figure 3.13A). The protein levels of TIM23 constituents TIM21, TIM23, TIM50 were not affected in TAZ KO cells. The core components of the TOM complex TOM40 and TOM70 were not altered as well. The TIM22 complex constituent acylglycerol kinase (AGK) is involved in generation of phosphatidic acid (PA), a precursor of cardiolipin biosynthesis (Vukotic et al., 2017). AGK has a dual function as a TIM22 complex scaffold protein and it phosphorylates mono- and diacylglycerols in phospholipid biosynthesis. The protein steady state levels of AGK were examined, to address, if the lack of TAZ affected upstream cardiolipin biosynthesis pathway. However, TIM22 core components TIM22 and AGK levels were also not altered in TAZ KO (Figure 3.13A). The steady state levels of translocase complexes were examined by 2D-BN-SDS-PAGE analysis (Figure 3.13C). This method gave indications if lacking tafazzin affected the structural integrity of translocases. The core complex of TIM23 $(100 \mathrm{kDa})$ and the core complexes of TOM and TIM22 (400 kDa) were not affected in TAZ KO mitochondria. The maintenance of protein complexes indicated that core constituents were not dissociated from interaction partners. This finding was confirmed by coimmunprecipitation of TIM23 and TIM50. The interaction of both core components in TAZ KO MEF cells was not affected (Figure 3.13B).

These approaches indicate, that tafazzin deficiency in MEF cells does not affect translocases in MEF cells. 
A
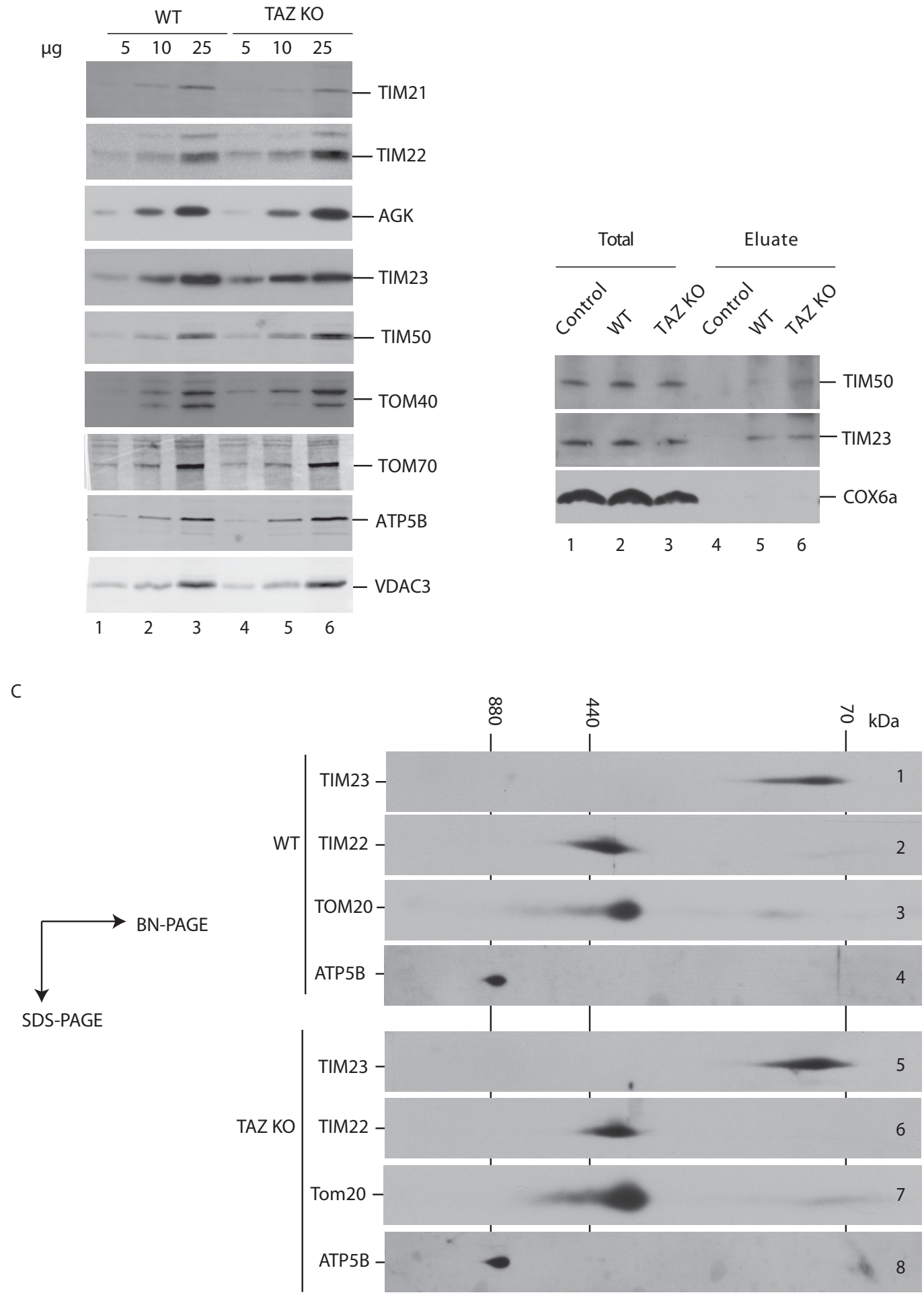

Figure 3.13: The protein levels and structural integrity of translocases are not affected in TAZ KO MEF mitochondria

(A) Mitochondria from WT and TAZ KO MEF cells were isolated by differential centrifugation and separated on a $12,5 \%$ SDS-PAGE resolving gel. Indicated proteins were detected by immunoblotting.

(B) Mitochondria from WT MEF cells were coimmunprecipitated by TIM23 and control antibody (control) in 1\% digitonin-containing solubilization buffer. Samples were separated on 12,5\% SDSPAGE running gel and immunoblotted against TIM50, TIM23 and COX6a 
(C) WT and TAZ KO MEF mitochondria were separated by 2D-BN-SDS-PAGE analysis. Protein complexes were detected by western blotting against TIM50, TIM23 and COX6a

\subsubsection{MCU and EMRE are presequence-containing mitochondrial proteins}

The reorganized MCU complexes in TAZ KO MEF cells suggested, that the assembly of MCU complexes were affected. Therefore, the import and assembly pathway of

$\mathrm{MCU}$ complexes via in-vitro import of ${ }^{35} \mathrm{~S}$ labeled MCU and EMRE precursors into isolated mitochondria was addressed. The precursors were translated from mRNA in reticulocyte lysates. Both, MCU (Figure 3.14A) and EMRE (Figure 3.14B) contained a mitochondrial presequence, which was cleaved after protein translocation into mitochondria. The presequence of MCU consisted of 50 amino acids (total weight: 39 $\mathrm{kDa}$ and presequnce: $5 \mathrm{kDa}$ ) and EMRE contained a presequence of $4 \mathrm{kDa}$ (total weight: $11 \mathrm{kDa}$ ). Newly synthetized MCU and EMRE preproteins were imported in a time-dependent manner. A cocktail of antimycin, valinomycin and oligomycin (AVO mix) dissipated the membrane potential, the driving force of mitochondrial import, thus acted as a negative control. The treatment of mitochondrial samples with proteinase $\mathrm{K}(\mathrm{PK})$ after import reactions showed that the cleaved and matured forms of MCU and EMRE were imported to PK-protected mitochondrial compartments. The non-imported precursors were degraded by PK treatment. The degradation products of the EMRE precursor covered the matured EMRE, so that EMRE import reactions in following chapters were performed without PK treatments.

A protocol of in-vitro import reactions of MCU and EMRE precursors was extablished for further analysis of MCU assembly. 
A

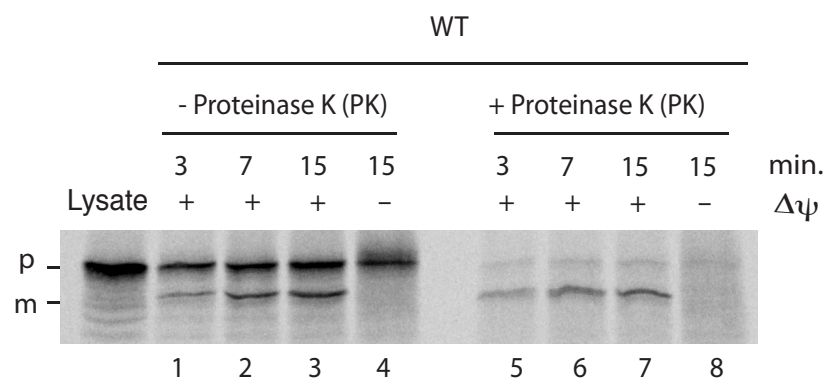

B

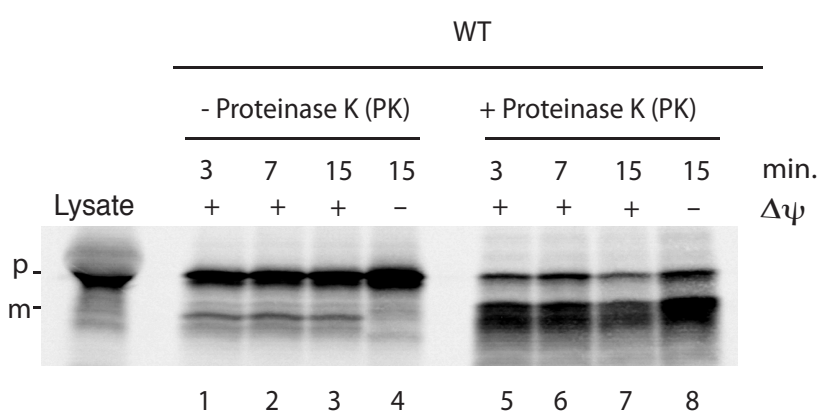

Figure 3.14: Radiolabeled human $\left[{ }^{35} \mathrm{~S}\right] \mathrm{MCU}$ and $\left[{ }^{35} \mathrm{~S}\right]$ EMRE preproteins contain a mitochondrial presequence, which is cleaved after in-vitro import into isolated mitochondria

(A) $\left[{ }^{35} \mathrm{~S}\right] \mathrm{MCU}$ and (B) $\left[{ }^{35} \mathrm{~S}\right]$ EMRE were imported into freshly prepared WT MEF mitochondria for depicted time points. Import reactions were stopped by membrane potential dissipation. Samples were treated with proteinase $\mathrm{K}(\mathrm{PK})$ where indicated. Import samples were analysed by SDS-PAGE analysis, additional western blotting and autoradiography. p, precursor; m, mature protein.

\subsubsection{Import of radiolabeled EMRE and motor-dependent import of OTC into MEF mitochondria is not affected by TAZ KO}

The mitochondrial precursor import pathway in purified TAZ KO MEF mitochondria can be tested by in-vitro import of newly synthezised mitochondrial precursors. The soluble mitochondrial protein ornithine transcarbamylase (OTC) has been used to examine if the motor- and TIM23-dependent protein translocation into TAZ KO mitochondria was affected. The import of newly synthetized radioactive OTC into isolated TAZ KO mitochondria was not changed (Figure 3.15A+B).

The EMRE protein steady state levels were not affected in TAZ KO MEF cells (Figure 3.1). This observation could correlate with unaltered mitochondrial import of EMRE preproteins. Indeed, ${ }^{35} \mathrm{~S}$ labeled EMRE import into WT and TAZ KO MEF mitochondria were not affected (Figure 3.15C+D).

In-vitro import of the mitochondrial preproteins EMRE and OTC is not altered in TAZ KO MEF mitochondria. 

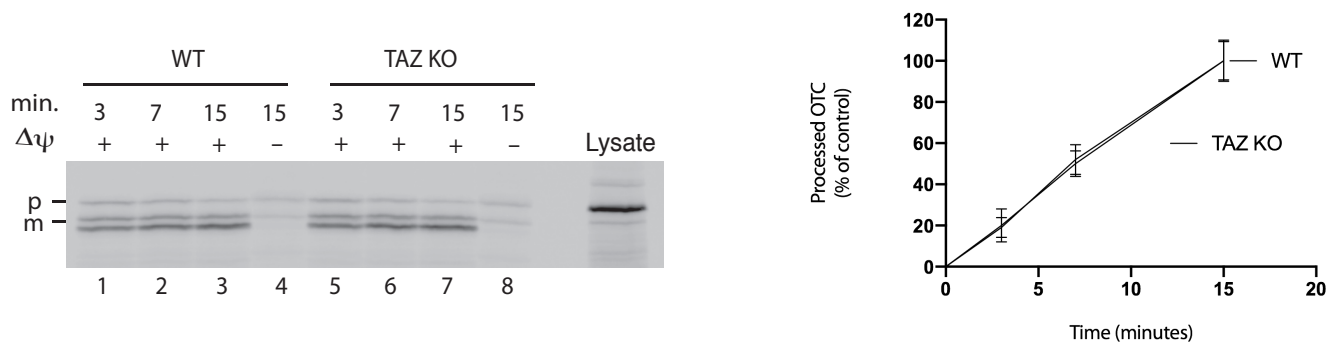

C

D
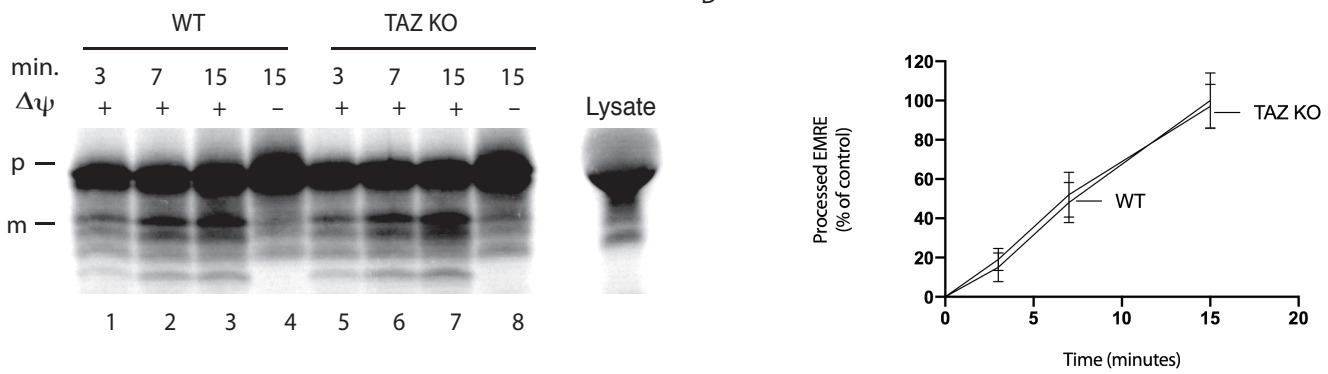

Figure 3.15: In-vitro import of $\left[{ }^{35} \mathrm{~S}\right]$ OTC from rat and human $\left[{ }^{35} \mathrm{~S}\right]$ EMRE preproteins into isolated MEF mitochondria are not affected in TAZ KO

(A) $\left[{ }^{35} \mathrm{~S}\right]$ OTC and (C) $\left[{ }^{35} \mathrm{~S}\right]$ EMRE were imported into freshly prepared WT and TAZ KO MEF mitochondria. Import reactions were stopped by membrane potential dissipation. Samples of $\left[{ }^{35}\right.$ S $]$ OTC import were treated with proteinase K (PK). Import samples were analysed by SDS-PAGE analysis, additional western blotting and autoradiography

(B) $\left[{ }^{35} \mathrm{~S}\right]$ OTC and (D) $\left[{ }^{35} \mathrm{~S}\right]$ EMRE imports were quantified densiometrically by ImageJ ( $\mathrm{n}=3$, mean \pm SEM). p, precursor; $\mathrm{m}$, mature protein.

\subsubsection{Interaction of newly imported radioactive EMRE with MCU-FLAG is not affected in TAZ KO MEF mitochondria}

The in-vitro import of EMRE preprotein was unaffected in TAZ KO MEF mitochondria (Figure 3.15) and the assembly of newly imported EMRE into MCU complex was addressed. The interaction of in-vitro imported EMRE with MCU was investigated by coimmunoprecipitation (Co-IP) of radioactive, newly imported EMRE with FLAG-tagged MCU of MCU-FLAG-overexpressing WT and TAZ KO MEF mitochondria. EMRE precursors were imported for 30 minutes instead of 15 minutes to enable its insertion into the inner mitochonrial membrane and assembly with MCU. Afterwards, these mitochondria were solubilized by digitonin and were subjected to coimmunpreticipation with FLAG-tagged MCU. The input samples of the coimmunprecipitation confirmed that the EMRE import into WT and TAZ KO mitochondria were not affected (Figure 3.16A). $50 \%$ of the Co-IP eluate was loaded 
on SDS-PAGE. The eluate showed how much of imported EMRE was pulled down in WT and TAZ KO. The interaction of newly imported EMRE with MCU-FLAG revealed no differences between both genotypes. The other $50 \%$ of the eluate were loaded on BN-PAGE to analyse, in which MCU-FLAG-containing complexes EMRE was assembled (Figure 3.16B). EMRE assembly into MCU underwent a ratio shift between WT and TAZ KO mitochondria. EMRE assembled more to the $1000 \mathrm{kDa}$ MCU complex in WT mitochondria while the majority of EMRE in TAZ KO assembled with the the $500 \mathrm{kDa}$ MCU complex. This observation confirmed the previous findings that MCU-EMRE complex organization was characterized by a ratio shift of MCU complexes in the $\mathrm{kDa}$ range (Figure 3.3) that the MCU-EMRE interaction was not affected in TAZ KO MEF cells (Figure 3.6).

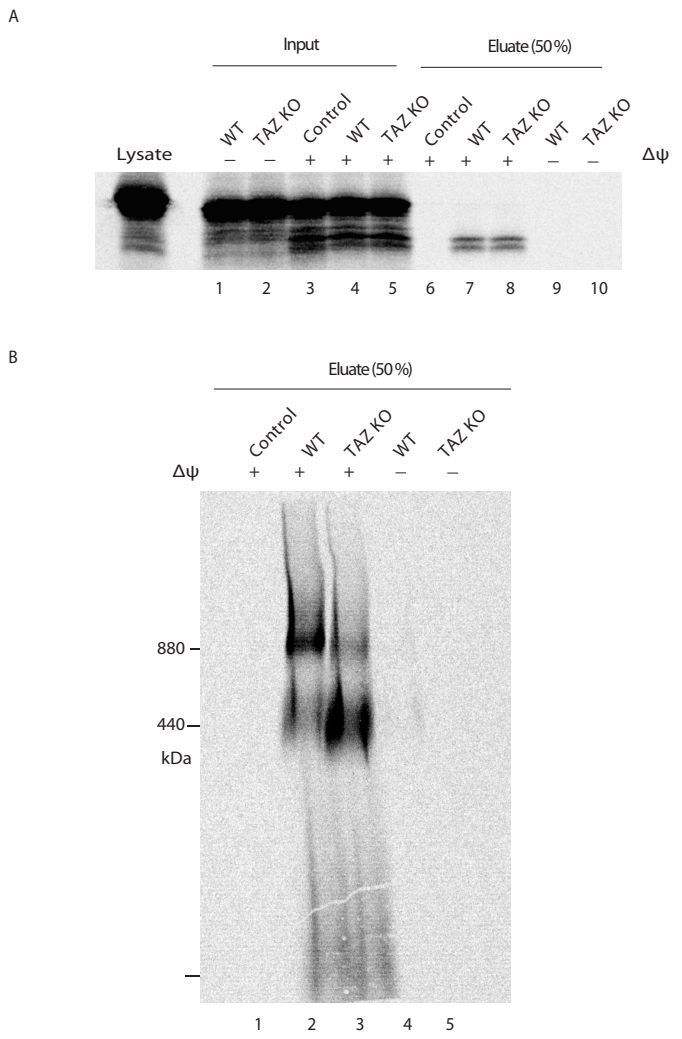

Figure 3.16: Interaction of newly imported $\left[{ }^{35} \mathrm{~S}\right] \mathrm{EMRE}$ with $\mathrm{MCU}$ is not altered in TAZ KO MEF cells

(A) Human $\left[{ }^{35} \mathrm{~S}\right]$ EMRE preproteins were imported into MCU-FLAG-containing WT and TAZ KO MEF mitochondria which were transfected with human MCU-FLAG plasmid three days before. Imports into freshly prepared WT and TAZ KO MEF mitochondria were conducted for 30 minutes. Import reactions were stopped by membrane potential dissipation. Mitochondria were solubilized by $1 \%$ digitonin-containing buffer and coimmunprecipitated by FLAG beads. $50 \%$ of the native eluate was loaded on SDS-PAGE and (B) $50 \%$ to BN-PAGE analysis. $\left[{ }^{35} \mathrm{~S}\right.$ ] EMRE import and assembly samples were analysed by SDS-PAGE analysis and autoradiography. p, precursor; m, mature protein. 


\subsubsection{In-vitro precursor import of radiolabeled MCU into isolated WT and TAZ KO MEF mitochondria}

In addition to the in vitro import and assembly of EMRE preproteins into MEF mitochondria, MCU precursor imports were established to study EMRE-MCU interaction. Therefore, the human MCU preproteins synthesized reticulocyte lysate were imported to WT and TAZ KO MEF mitochondria. Controversely, the amount of matured $\mathrm{MCU}$ in the TAZ KO import sample was decreased (Figure 3.17A+B). MCU protein steady state levels were unaltered (Figure 3.1). The time frame of the MCU import did not matter. The reduction of processed MCU in TAZ KO was observed in all time points.

A

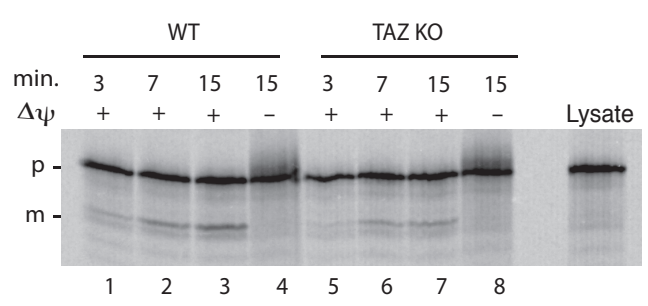

B

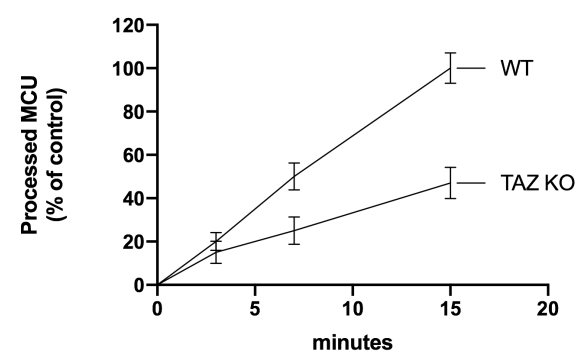

Figure 3.17: In-vitro import of $\left.{ }^{35} \mathrm{~S}\right] \mathrm{MCU}$ preproteins into isolated MEF mitochondria is affected in TAZ KO

(A) Human $\left[{ }^{35} \mathrm{~S}\right] \mathrm{MCU}$ was imported into freshly prepared WT and TAZ KO MEF mitochondria. Import reactions were stopped by membrane potential dissipation. Import samples were analysed by SDS-PAGE analysis, additional western blotting and autoradiography.

(B) $\left[{ }^{35} \mathrm{~S}\right] \mathrm{MCU}$ imports were quantified densiometrically by ImageJ $(\mathrm{n}=3$, mean $\pm \mathrm{SEM}) . \mathrm{p}$, precursor; $\mathrm{m}$, mature protein.

\subsubsection{EMRE interaction with imported MCU is not affected in TAZ KO MEF cell mitochondria}

The levels of mature MCU after in-vitro import of MCU preprotein into TAZ KO MEF mitochondria were reduced (Figure 3.17). The effect of reduced mature MCU on assembly of newly imported EMRE into MCU complex was addressed. The interaction of newly imported MCU with endogenous EMRE in WT and TAZ KO mitochondria has been investigated by Co-IP. Radioactive labeled MCU was imported to isolated mitochondria for 30 minutes and solubilized by digitonin. MCU were pulled down by EMRE antibodies coated to protein A sepharose. The input 
samples of the EMRE antibody IP showed the import reactions as well (Figure 3.18A, lanes 4 and 5). The matured MCU was reduced in comparison to imported MCU in WT. The eluate samples revealed that endogenous, pulldowned EMRE interacted with imported MCU. The interaction of MCU and EMRE seemed to be reduced in TAZ KO, but the signal of matured/imported MCU in TAZ KO was reduced to the same degree (around 78\%, Figure 3.18B). As a consequence, the amount of matured MCU in TAZ KO was decreased, so that MCU could just interact with endogenous EMRE in a lesser amount. Interaction of endogenous EMRE with imported radioactive MCU is not affected in TAZ KO MEF cell mitochondria.

A

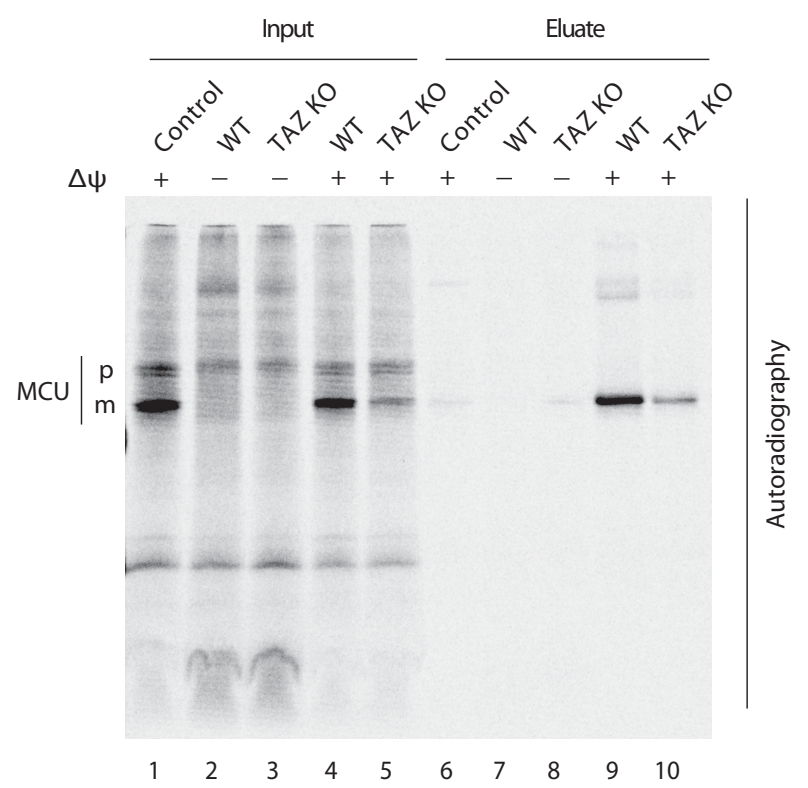

B

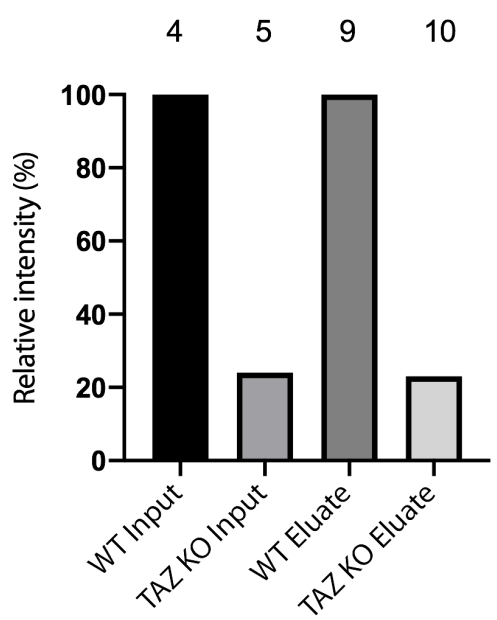

Figure 3.18: Interaction of newly imported $\left[{ }^{35} \mathrm{~S}\right] \mathrm{MCU}$ with endogenous EMRE is not altered in TAZ KO MEF cells

(A) Human $\left.{ }^{35} \mathrm{~S}\right] \mathrm{MCU}$ preproteins were imported into WT and TAZ KO MEF mitochondria. Imports into freshly prepared WT and TAZ KO MEF mitochondria were performed for 45 minutes. Import reactions were stopped by membrane potential dissipation. Mitochondria were solubilized by $1 \%$ digitonin-containing buffer and coimmunprecipitated by EMRE co-immunpreticipation (Co-IP). Tafazzin antibody acted as negative control. Import and Co-IP samples were loaded and separated on $10-18 \%$ gradient SDS-PAGE. $\left[{ }^{35}\right.$ S]MCU import and Co-IP samples were analysed by SDS-PAGE analysis, additional western blotting and autoradiography. $\mathrm{p}$, precursor; $\mathrm{m}$, mature protein.

(B) Experiment was performed twice, quantified by ImageJ and showed the same result $(n=2)$.

\subsubsection{Turnover of newly imported MCU is unchanged in WT and TAZ KO MEF}

\section{mitochondria}


The reduced amount of matured MCU in TAZ KO MEF mitochondria after in vitro import did not affect the EMRE-MCU interaction (Figure 3.18). Further analysis was required to investigate, why matured $\mathrm{MCU}$ was reduced. One possibility was a pulse/chase assay to investigate the turnover of imported MCU in WT and TAZ KO MEF mitochondria.

The hypothesis was, that the turnover of imported MCU could have been decreased in TAZ KO. MCU was imported for 15 minutes and the reduction of processed MCU in TAZ KO mitochondria was observed (Figure 3.19A, lanes 2 and 7). The import reactions were stopped by dissipating the membrane potential via adding AVO mix. In the following hour, samples were taken at the depicted time points. The turnover of matured MCU in WT and TAZ KO was unaffected (Figure 3.19B). Figure 3.17 gave already an indication that the reduced processed MCU levels in TAZ KO did not depend on the import time frame. Longer import could provoke higher turnover of MCU in TAZ KO perhaps. However, MCU import after 3, 7 and 15 minutes were decreased in comparison to WT (Figure 3.17), so that the import time of 15 minutes was not too long.

The degradation rate of newly imported, mature MCU is not altered in WT and TAZ KO MEF mitochondria.

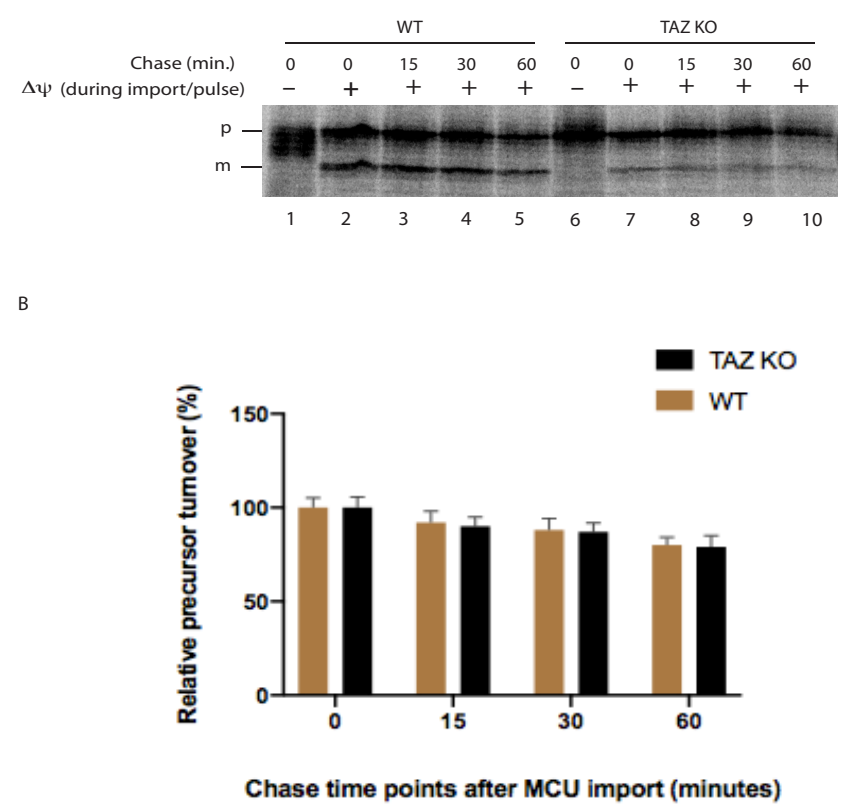


Figure 3.19: Turnover of newly imported $\left[{ }^{35} \mathrm{~S}\right] \mathrm{MCU}$ in TAZ KO MEF mitochondria is not affected

(A) Human $\left[{ }^{35} \mathrm{~S}\right] \mathrm{MCU}$ preproteins were imported into WT and TAZ KO MEF mitochondria for 15 minutes (Pulse). Import reactions were stopped by membrane potential dissipation. Single import samples were taken after 15, 30 and 45 minutes (Chase). Import samples were loaded and separated on $12,5 \%$ SDS-PAGE resolving gels. Separated samples were blotted to PVDF membranes and exposed to phosphor screens (autoradiography). p, precursor; $\mathrm{m}$, mature protein.

(B) $\left[{ }^{35} \mathrm{~S}\right] \mathrm{MCU}$ import and turnover samples were quantified densiometrically by ImageJ $(\mathrm{n}=3$, mean \pm SEM).

\subsubsection{Insertion of proteins containing 2 transmembrane domains (TMDs) is not affected in TAZ KO}

The MCU protein contains two transmembrane domains (TMDs) and insertion of both TMDs into the IMM was affected in TAZ KO mitochondria. This hypothesis was tested by an in-vitro import of yeast Pam17, which contains two TMDs as well. Pam17 import and maturation were not reduced in comparison to MCU import in TAZ KO. Mature Pam17 was even increased (Figure 3.20). Two-transmembrane domains did not seem to be the reason for the reduced amout of mature MCU in TAZ KO MEF mitochondria (Figure 3.17).

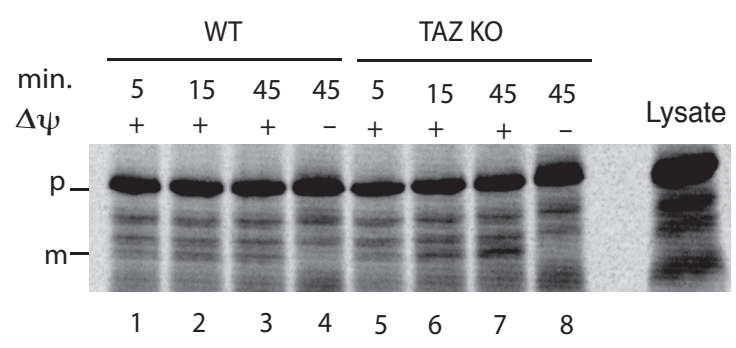

Figure 3.20: In-vitro import of yeast $\left.{ }^{35} \mathrm{~S}\right] \mathrm{Pam} 17$ preproteins into isolated MEF mitochondria is not reduced in TAZ KO in comparison to imported, mature $\mathrm{MCU}$

Yeast $\left[{ }^{35} \mathrm{~S}\right]$ Pam17 was imported into freshly prepared WT and TAZ KO MEF mitochondria. Import reactions were stopped by membrane potential dissipation. Import samples were analysed by SDSPAGE analysis, additional western blotting and autoradiography.

The experiment was performed two times with the same result $(n=2)$ p, precursor; m, mature protein.

\subsubsection{Lack of MICU1 does not affect MCU in-vitro import}

Figure 3.7 indicated, that TAZ KO MEF mitochondria contain less MICU1 bound to the MCU pore. The MICU1 steady state levels were not reduced (Figure 3.1), but the MDa MCU complex (MCU-3) in TAZ KO was lacking. The reduction of one MCU constituent on the complex, here MICU1, could influence the import of MCU. 
Therefore, MICU1 mRNA levels were silenced by specific siRNA for three days and MCU was imported into non-targeted and MICU1 knockdown MEF mitochondria (Figure 3.21). However, MICU1 knockdown did not affect MCU protein steady state levels or the in-vitro import of MCU.

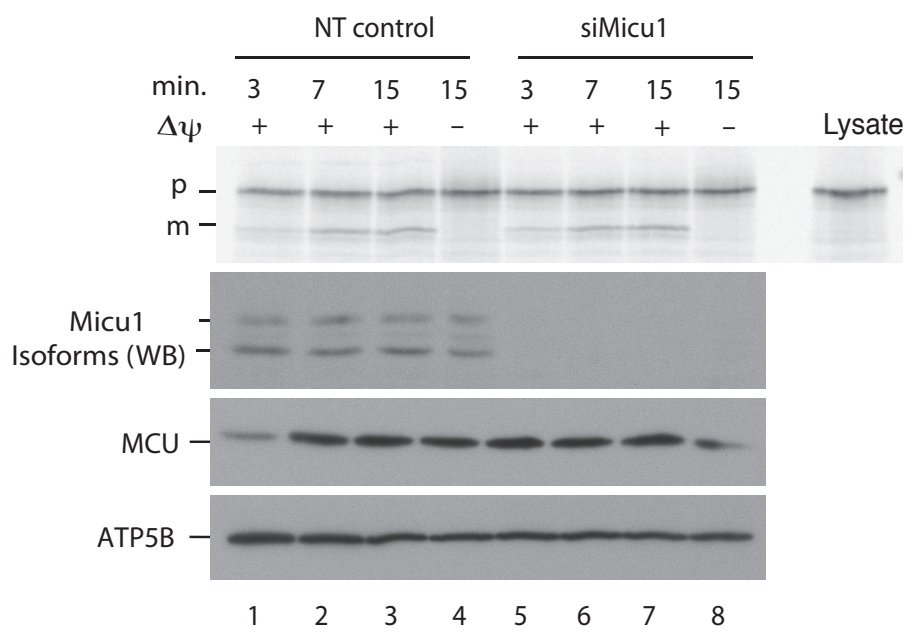

Figure 3.21: In-vitro import of $\left[{ }^{35} \mathrm{~S}\right] \mathrm{MCU}$ preproteins into Micu1-silenced MEF mitochondria is not affected

(A) Human $\left[{ }^{35} \mathrm{~S}\right] \mathrm{MCU}$ was imported into freshly prepared non-target control (NT) and MICU1 knockdown MEF mitochondria (siMICU1) 3 days after siRNA tranfection. Import reactions were stopped by membrane potential dissipation. Import samples were analysed by SDS-PAGE analysis, additional western blotting and autoradiography.

Membrane was immunoblotted with antibodies against MCU, MICU1 and ATP5B. p, precursor; m, mature protein.

\subsubsection{Membrane potential sensitivity of MCU import in TAZ KO mitochondria}

Chowdhury et al. already published, that the membrane potential of TAZ KO MEF mitochondria is reduced (Chowdhury et al., 2018). The mitochondrial membrane potential is the driving force of mitochondrial preprotein import. Decreased amount of membrane potential could impact the protein import in general or only for single precursors. Figure 3.15 has already shown, that EMRE and OTC import into TAZ KO MEF mitochondria was not reduced. Hence, the dampened membrane potential in TAZ KO MEF mitochondria did not affect the mitochondrial protein translocation of these two preproteins. However, the possibility was addressed, if mitochondrial import of MCU preprotein was more sensitive to the reduced membrane potential in TAZ KO MEF mitochondria (Figure 3.22). Therefore, radiolabeled MCU precursor was imported into CCCP-treated MEF mitochondria (CCCP: Carbonylcyanid-m- 
chlorphenylhydrazon). Final CCCP concentrations in mitochondria were titrated from 0 to $400 \mathrm{nM}$. MCU precursor was added to every single import reaction 3 minutes after adding CCCP. The motor-dependent OTC import was not affected TAZ KO MEF mitochondria (Figure 3.22A, lanes 2 and 8). Upon increasing CCCP concentrations, OTC import was reduced (Figure 3.22B). Interestingly, MCU preproteins could just imported upon $50 \mathrm{nM} \mathrm{CCCP}$, indicating that $\mathrm{MCU}$ import could require more membrane potential to be translocated to its mitochondrial localization. The direct comparison of OTC and MCU imports into CCCP-treated WT and TAZ KO mitochondria was performed once (Figure 3.22A). However, the comparison of radioactive OTC and MCU imports into CCCP-treated WT mitochondria was performed several times and was quantified. The reduced MCU import into membrane potential depleted mitochondria was statistically relevant (Figure 3.22B). The reduced amount of mature MCU could be explained by reduced membrane potential in TAZ KO MEF mitochondria.
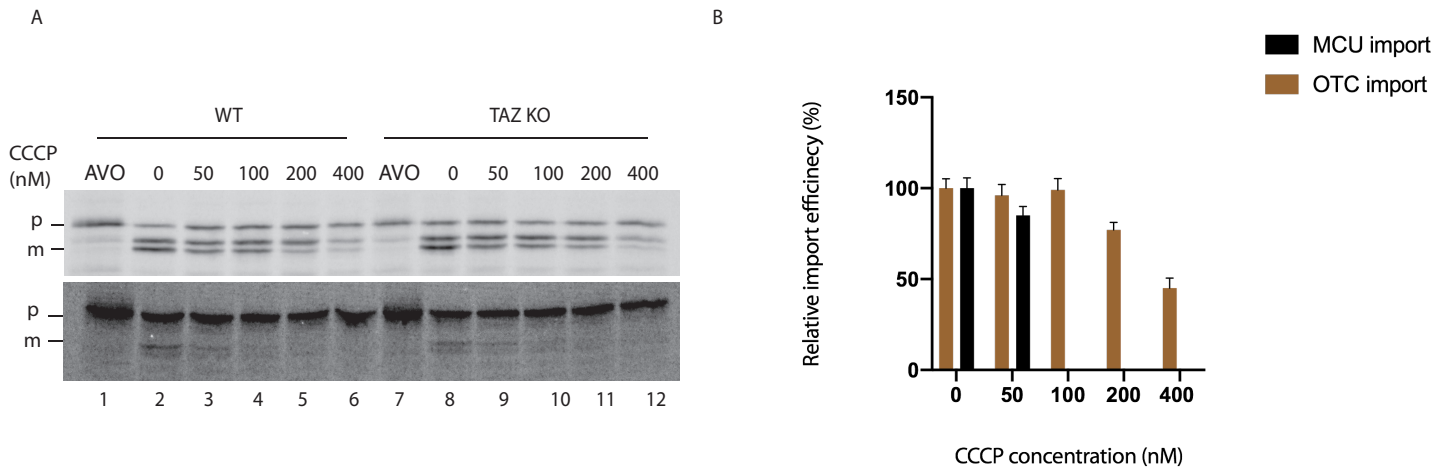

Figure 3.22: In-vitro import of $\left[{ }^{35} \mathrm{~S}\right]$ MCU preproteins is membrane potential sensitive in comparison to $\left[{ }^{35} \mathrm{~S}\right]$ OTC import into WT MEF mitochondria

(A) Human $\left[{ }^{35} \mathrm{~S}\right] \mathrm{MCU}$ and rat $\left[{ }^{35} \mathrm{~S}\right]$ OTC were imported into freshly prepared WT and TAZ KO MEF mitochondria for 15 minutes. 3 minutes, before starting each import reaction, CCCP (Carbonylcyanid$m$-chlorphenylhydrazon) in the indicated concnetrations was added. Import reactions were stopped by membrane potential dissipation. Import samples were analysed by SDS-PAGE analysis, additional western blotting and autoradiography.

$\mathrm{p}$, precursor; $\mathrm{m}$, mature protein.

(B) The comparison of $\left[{ }^{35} \mathrm{~S}\right] \mathrm{MCU}$ and $\left[{ }^{35} \mathrm{~S}\right]$ OTC import into WT cells was performed three times and was quantified by ImageJ $(n=3$, mean \pm SEM).

\subsection{MCU complexes in different Barth syndrome mouse organs}




\subsubsection{MCU complexes are tissue-specifically organized}

The finding of altered MCU complex organization in TAZ KO MEF cells brought up the question, how MCU complexes are composed or organized in other taffazindeficient model systems, such as organs from the ROSA26 shTAZ mouse (Barth Syndrome (BTHS) mouse). The tafazzin silencing in the BTHS mouse was caused by a conditional doxycyclin-dependent shTAZ expression.

To address the MCU complex organization in mouse organs, isolated mitochondria from different tissues were solubilized in a $1 \%$ digitonin-containing solution and separated on BN-PAGE in the first and on SDS-PAGE in the second dimension. Figure 3.23 displays the direct comparison of the migration pattern of $\mathrm{MCU}$ complexes in WT/TAZ KO MEF cells, WT/BTHS mouse skeletal muscle, WT liver and WT brain (Figure 3.23). The ratio shift of the $1 \mathrm{MDa}$ (MCU-2, lane 2) and 500 $\mathrm{kDa}$ (MCU-3, lane 2) MCU complexes in MEF cells were observed for WT and BTHS skeletal muscle MCU as well. After BN-PAGE separation MCU from skeletal muscle and MEF cells displayed the same migration pattern in both genotypes, respectively. MEF cell mitochondria contained the higher MDa MCU complex (MCU-1, lane 2), which was not present in skeletal muscle cells. The migration pattern of mouse skeletal muscle and MEF MCU complexes on BN-PAGE differ from the migration pattern of mouse liver and brain MCU. The predominant MCU form in mouse liver migrated at $400 \mathrm{kDa}$ and was smaller than the WT MCU complexes in MEF cells and skeletal muscle. In contrast, WT mouse brain MCU complexes occured in the MDa range, predominantly (Figure 3.23).

The direct comparison of separated MCU complexes displayed, that MCU complexes are differently organized in mouse organs. 


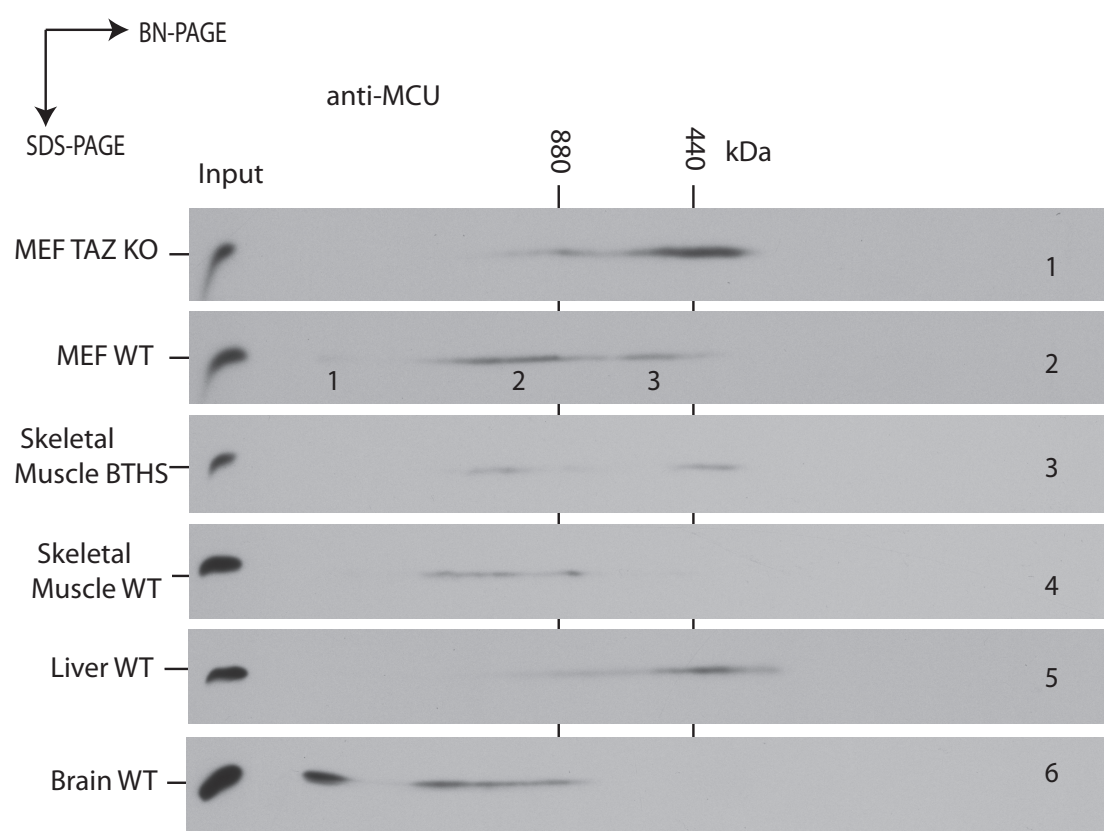

Figure 3.23: MCU complex organization is tissue- and cell type-specific.

Mitochondria isolated from WT and BTHS mouse liver, brain, skeletal muscle and MEF cells were solubilized by $1 \%$ digitonin-containing buffer and resolved by $2 \mathrm{D}-\mathrm{BN}-\mathrm{SDS}-\mathrm{PAGE}$ analysis. Protein complexes were detected by immunoblotting against MCU and ATP5B

\subsubsection{Protein steady state levels of respiratory chain components and MCU in mouse skeletal muscle}

Respiratory chain and MCU steady state levels in BTHS mouse skeletal muscle mitochondria were characterized. It is already known, that complex II levels are reduced and the respiratory chain complexes are remodeled in BTHS mouse hearts (Dudek et al., 2016). These findings were cardiac specific and were not found in liver or kidney. The protein steady state levels of CI, CIII, CIV and CV components were not affected in BTHS mouse hearts and patient-derived iPSC cardiomyocytes (Dudek et al., 2016). Thus, we monitored, if protein levels of the respiratory chain showed alterations in BTHS mouse skeletal muscle. A deeper look into the protein levels of mitochondrial proteins COX1, RIESKE and ATP5B (Figure 3.24A) showed, that tafazzin deficiency in mouse skeletal muscle did not alter the protein abundance of these core constituents. Figure 3.24B displays, that respiratory chain complexes were reorganized, which was already shown in BTHS cardiomyocytes (Dudek et al., 2016). CII (SDHA decoration) was not significantly reduced in BTHS skeletal muscle in comparison to BTHS mouse hearts. Respirasomes in BTHS skeletal muscle were reduced and CI and CIII were enriched in a mutual complex, which is also known 
from other BTHS models (Dudek et al., 2016). Interestingly, MCU and its interaction partner EMRE were slightly reduced in BTHS skeletal muscle mitochondria.

A

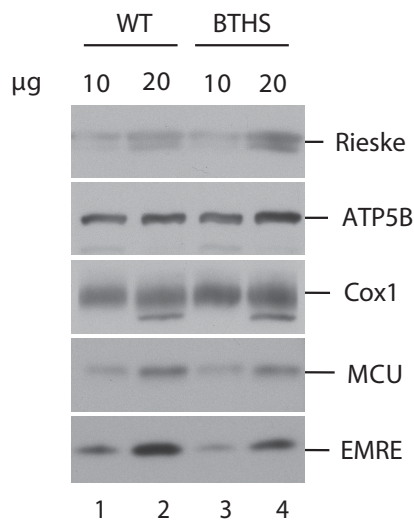

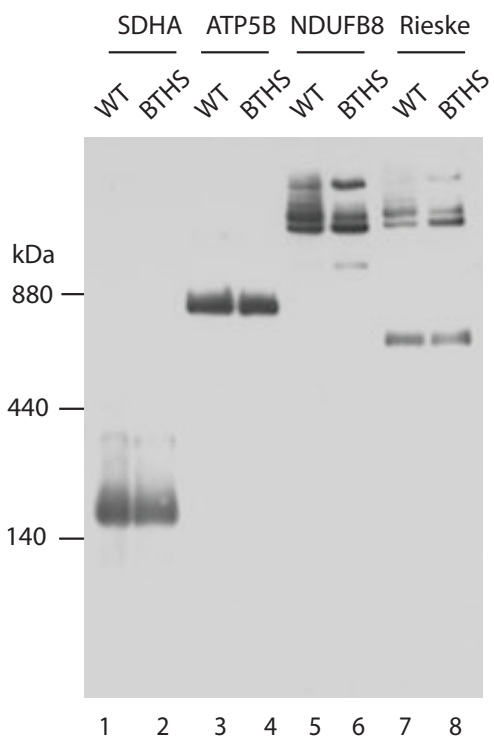

Figure 3.24: MCU and respiratory chain protein steady state levels in BTHS mouse skeletal muscle

(A) Mitochondria from WT and BTHS mouse skeletal muscle were isolated by differential centrifugation and separated on a $12,5 \%$ SDS-PAGE resolving gel. Indicated proteins were detected by immunoblotting $(\mathrm{n}=2)$

(B) Mitochondria from WT and BTHS mouse skeletal muscle were solubilized by $1 \%$ digitonin and separated on BN-PAGE resolving gel (4-13\%). Protein complexes were detected by immunoblotting against SDHA, NDUFB8, Rieske and ATP5B

\subsubsection{MCU/EMRE interaction in BTHS mouse skeletal muscle is unaffected}

The MCU complex ratio shifts in TAZ KO MEF cells and BTHS mouse skeletal muscle showed similarity in the migration pattern after 2D-BN-SDS-PAGE analysis. Furthermore, the comigration and interaction studies of MCU and EMRE in MEF cells have shown, that the MCU/EMRE interaction and steady state levels were unaffected in TAZ KO MEF cells. The interaction of MCU and EMRE in BTHS mouse skeletal muscle was addressed by coimmunprecipitation. MCU complexes comigrated with EMRE after 2D-BN-SDS-PAGE analysis (Figure 3.25A). MCU was pulled down by an EMRE antibody conjugated to protein-A sepharose (Figure 3.25B). The input of applied native lysates from WT and BTHS skeletal muscle mitochondria showed that MCU levels in BTHS skeletal muscle were slightly reduced 
in comparison to WT (Figure 3.25C, lanes 2 and 3). The levels of pulldowned MCU in BTHS (eluate) were decreased to the same extent as in the input (Figure 3.25C, lanes 5 and 6).

These results go along with the findings in MEF cells, that the integral MCU/EMRE module stays stable independently from tafazzin. MCU complexes in mouse skeletal muscle contain EMRE and the MCU/EMRE interaction in BTHS mouse skeletal muscle is unaffected. 
A

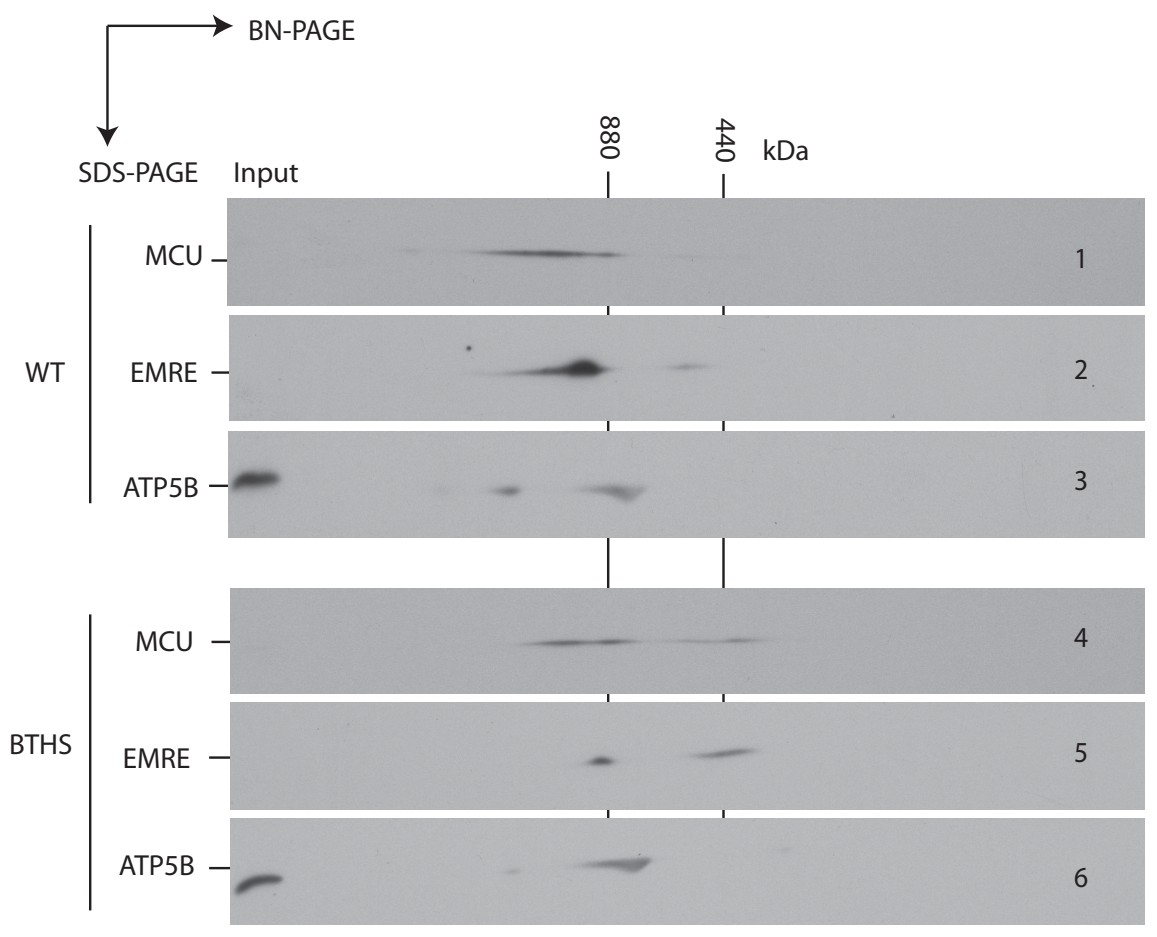

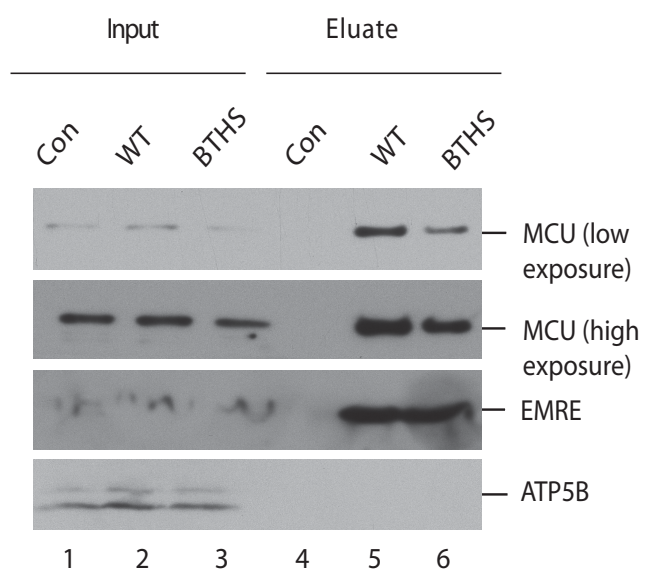

C

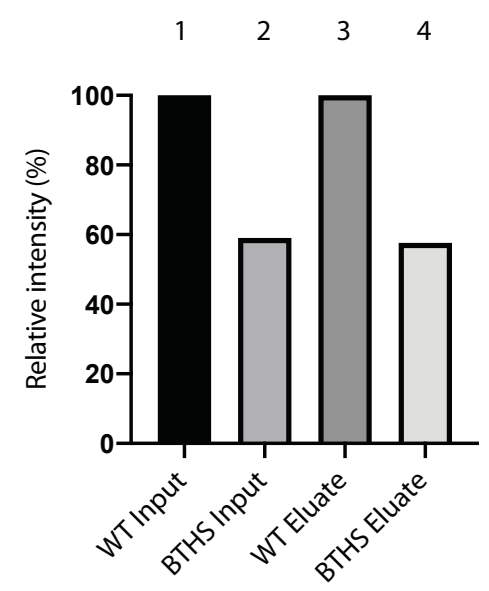

Figure 3.25: Lack of tafazzin causes reorganization of MCU complexes in mouse skeletal muscle, but does not affect MCU-EMRE interaction

(A) Mitochondria from WT and BTHS mouse skeletal muscle were solubilized by $1 \%$ digitonin and separated by 2D-BN-SDS-PAGE analysis. Indicated proteins were detected by immunoblotting.

(B) Mitochondria from WT and BTHS skeletal muscle were coimmunprecipitated by EMRE and tafazzin antibodies conjugated to protein A sepharose. Samples probed on SDS-PAGE were immunoblotted against EMRE, MCU and ATP5B

(C) Experiment (B) was quantified densiometrically by Image J $(\mathrm{n}=1)$, MCU bands in lanes 5 and 6 were normalized to the bait EMRE. 


\subsubsection{MCU and respiratory chain complexes in BTHS mouse brain are dissociated}

The composition of mouse brain MCU complexes and respiratory chain complex organization was investigated. MCU complexes and comigration of the interaction partner EMRE were detected after a subsequent second-dimensional run on SDSPAGE (Figure 3.26A). WT mouse brains express two major MCU-containing complexes in the MDa range (complexes MCU-1 and MCU-2), which are comparable to the MCU complex organisation in MEF cells (Figure 3.23). Both MCU-containing complexes comigrated with EMRE. MCU complexes in BTHS mouse brain underwent a ratio shift from the higher to the lower molecular weight forms. The MCU-1 oligomer was reduced and the MCU-2 complex became the more dominant form in BTHS brain. In addition, a lower molecular weight form of MCU at $500 \mathrm{kDa}$ (MCU-3) became more prominent (Figure 3.26A).

Mitochondria from WT and BTHS mouse brain were isolated and solubilized in $1 \%$ digitonin-containing buffer. Respiratory chain complexes were separated and visualized by BN-PAGE analysis in the first dimension (Figure 3.26B).

The respiratory chain complexes revealed a similar phenotype regarding the abundence of higher molecular weight forms in the MDa range. The respirasomes in BTHS brain mitochondria were reduced in comparison to WT. Particularly, CIV constituent COX6a resided in smaller CIV oligomers predominantly and much lesser in respiratory chain supercomplexes. The CIII dimer remained stabil in BTHS and CIand CIII-containing complexes comigrated (Figure 3.26B). Beside the alterations of OXPHOS complex organisation in BTHS brain mitochondria, the carrier translocase TIM22 and the metabolite- and ion-conducting pore VDAC3 were slightly reduced in BTHS mouse brains. 


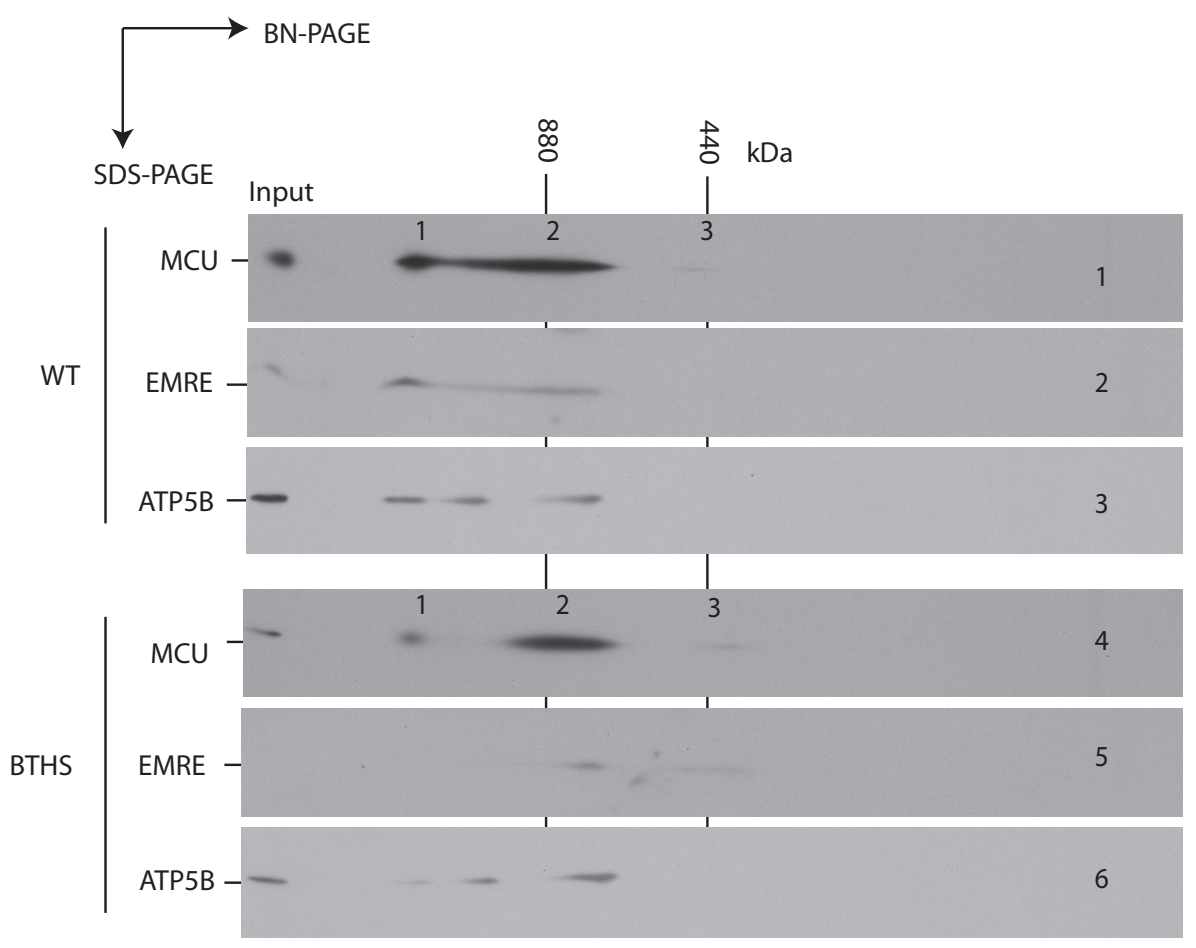

B

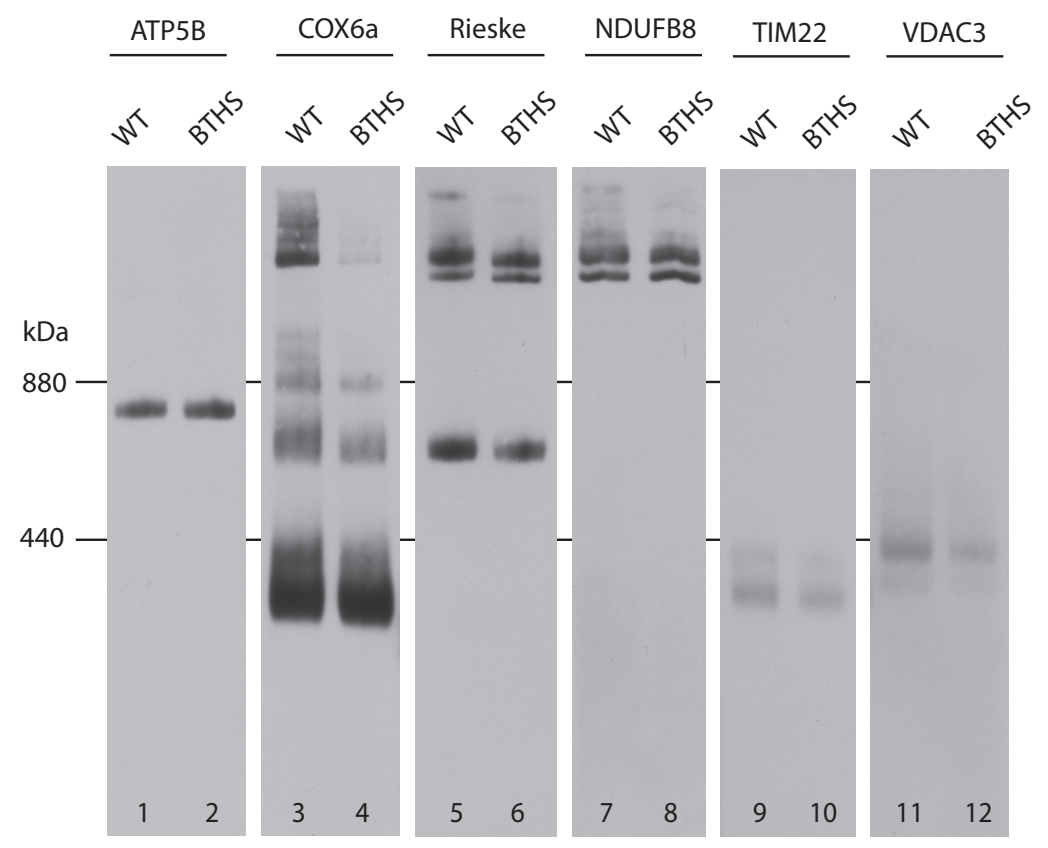

Figure 3.26: Lack of tafazzin causes reorganization of MCU and respiratory chain complexes

(A) Mitochondria from WT and BTHS mouse brains were solubilized by $1 \%$ digitonin and separated by 2D-BN-SDS-PAGE analysis. Indicated proteins were detected by immunoblotting.

(B) Mitochondria from WT and BTHS mouse brains were solubilized by $1 \%$ digitonin and separated by BN-PAGE analysis. Indicated proteins were detected by immunoblotting. 


\subsubsection{Tafazzin-deficiency does not affect MCU complex organization in BTHS mouse liver}

Figure 3.23 displayed, that MCU complexes undergo different organ-specific organizations. The MCU complex organization in WT and BTHS mouse liver was addressed. The predominant MCU complex in mouse liver mitochondria migrated at $400 \mathrm{kDa}$ in 2D-BN-SDS-PAGE analysis (Figure 3.27). Figures 3.3, 3.25, 3.26 revealed that higher molecular weight forms of MCU were more abundant in MEF cells, mouse brain and skeletal muscle. Possibly, the liver-specific cardiolipin species pool and metabolic demands define this phenotype. The lack of tafazzin expression did not seem to affect the stability of 400 - $500 \mathrm{kDa}$ MCU complexes in general, which was shown by the stability of the $500 \mathrm{kDa}$ MCU complexes in diverse BTHS models (Figures 3.3, 3.25, 3.26). This observation could explain that liver MCU complex integrity was not dependent on tafazzin activity. MCU complex steady state levels and organization were not affected in BTHS mouse liver.

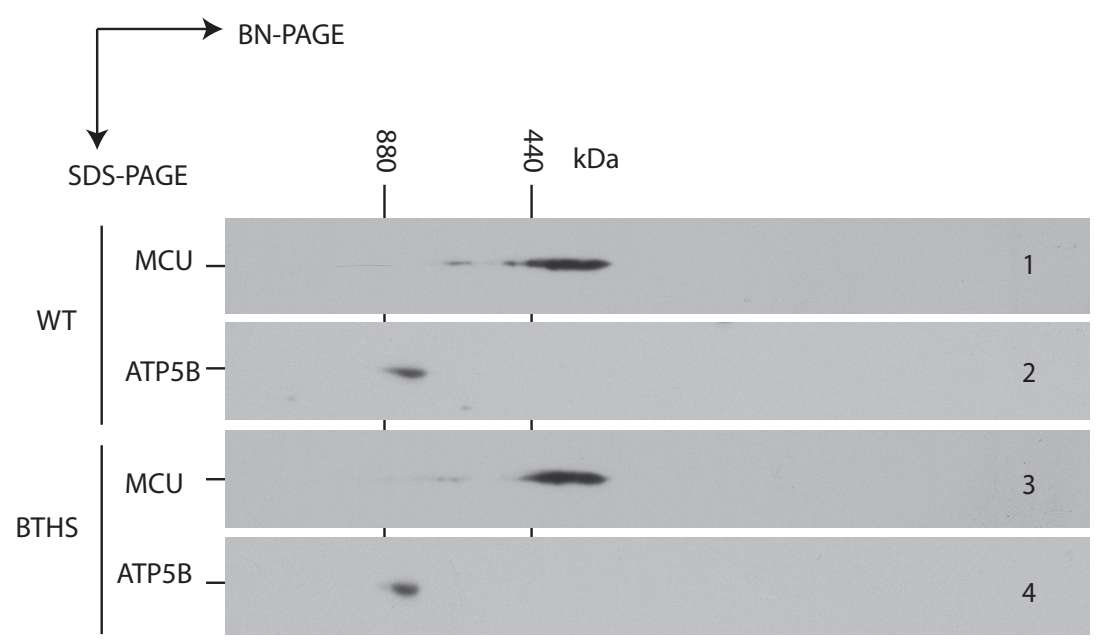

Figure 3.27: MCU complex organization in BTHS mouse liver is not affected Mitochondria isolated from WT and BTHS mouse liver were solubilized by $1 \%$ digitonin-containing buffer and resolved by 2D-BN-SDS-PAGE analysis. Protein complexes were detected by immunoblotting against MCU and ATP5B

\subsubsection{MCU protein steady state levels are reduced in mitochondria of BTHS mouse hearts}

BTHS mouse brain, skeletal muscle and TAZ KO MEF mitochondria showed tafazzin-dependent MCU complex dissociations. The MCU protein steady state levels 
in skeletal muscle were slightly reduced, but still abundant (Figure 3.24). The steady state levels of WT and BTHS mouse hearts were considered. Figure 3.28A displays the reduction of MCU protein levels in cardiac BTHS mitochondria. Mitochondria from 4 different WT and BTHS mice were isolated and the MCU protein levels were compared after SDS-PAGE separation. MCU levels were reduced in BTHS mice. The reduction or even loss of $\mathrm{MCU}$ could have consequences for cellular calcium homeostasis. Unpublished data indicated that mitochondrial calcium uptake into BTHS cardiomyocytes were depleted caused by the lack of MCU (Dudek, Maack et. al., unpublished). In addition, MCU mRNA levels in BTHS mouse hearts were not diminished (Rehling and Maack, unpublished).

The reduction of cardiac MCU in BTHS mice was compared to MCU steady state levels in both, mouse liver and brain. Both organs conduct relevant metabolic functions like heart and skeletal muscle. However, the MCU protein steady state levels in these organs were not decreased in BTHS mouse (Figure 3.28B). As a result, the reduction of MCU was heart-specific.

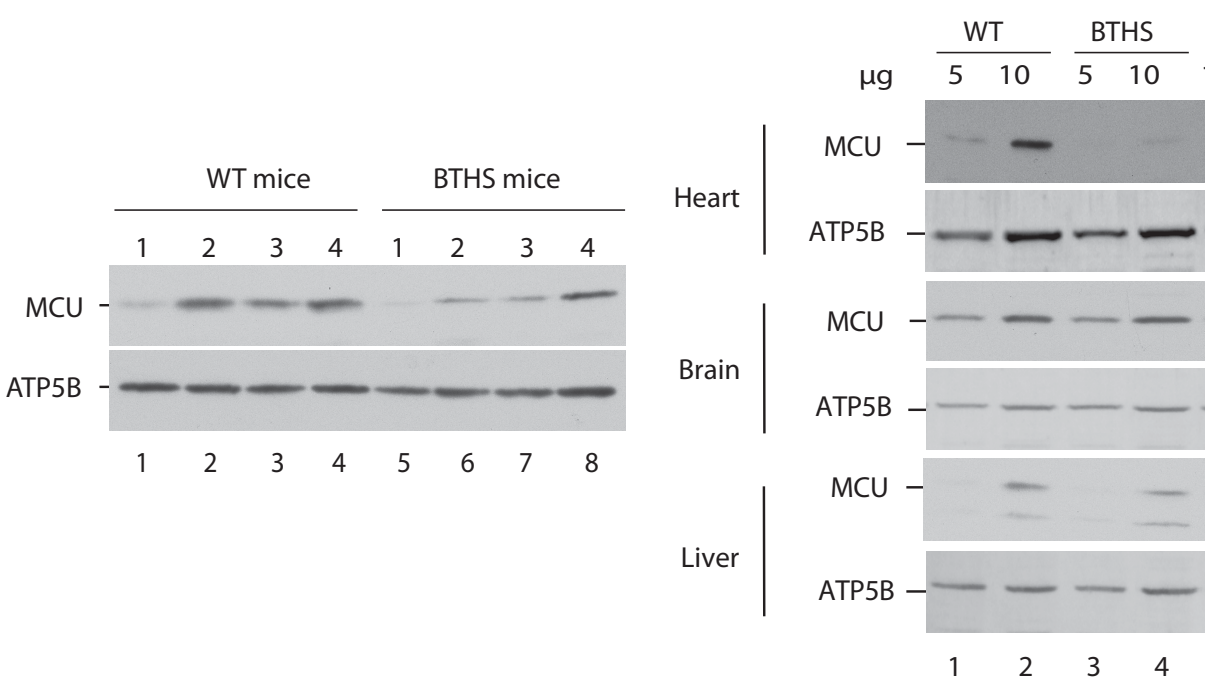

Figure 3.28: Reduction of MCU steady state levels in BTHS mouse hearts is not observed in liver and brain

(A) Cardiac mitochondria from four different WT and BTHS mice pairs were isolated by differential centrifugation and separated on a $12,5 \%$ SDS-PAGE resolving gel. Indicated proteins were detected by immunoblotting.

(B) Brain, liver and heart of a WT and BTHS mouse pair were dissected and mitochondria were isolated. Samples were separated on $12,5 \%$ SDS-PAGE running gel and immunoblotted against ATP5B and MCU 


\subsubsection{Steady state levels of MCU complexes are reduced in mitochondria of BTHS mouse hearts and BTHS patient derived cardiomyocytes}

To address to which extent the reduction of MCU in BTHS mouse hearts affect the steady state levels of MCU complexes, MCU/EMRE-containing complexes were separated via 1D- and 2D-BN-SDS-PAGE analysis. The loss of the MCU protein as one core component of the uniporter caused the loss of the total complex (Figure 3.29A). Reduced cardiac MCU complexes in BTHS did not show any indications for MDa complex dissociation or remodeling in comparison to the MCU complex ratio shifts in MEF TAZ KO cells, BTHS mouse skeletal muscle and brain. Furthermore, the respiratory chain complexes (here indicated as COX1/CIV decoration) were reorganized and respirasomes were reduced.

Further protein steady state levels of mitochondrial proteins in cardiac tissues were examined (Figure 3.30B). Respiratory chain components NDUFB8 (CI), RIESKE (CIII), COX4I1/2, COX6a (CIV) and ATP5B (CV) were not affected in BTHS mouse hearts as already published (Dudek et al., 2016). The levels of translocase constituents Tim22 and Tim50 were not altered, but LON protease 1 (LONP1) was increased in BTHS hearts. LONP1 participates in mitochondrial stress response and protein quality control (Li et al., 2019). Beside the reduction of MCU levels in BTHS cardiomyocytes (Figure 3.28), its interaction partners EMRE and MICU1 were slightly decreased as well, but not to the extent of the MCU reduction.

The reduction of MCU steady state levels in BTHS mouse hearts was also found in BTHS patient-derived induced pluripotent stem cell cardiomyocytes (iPSC cardiomyocytes) (Figure 3.29C). 


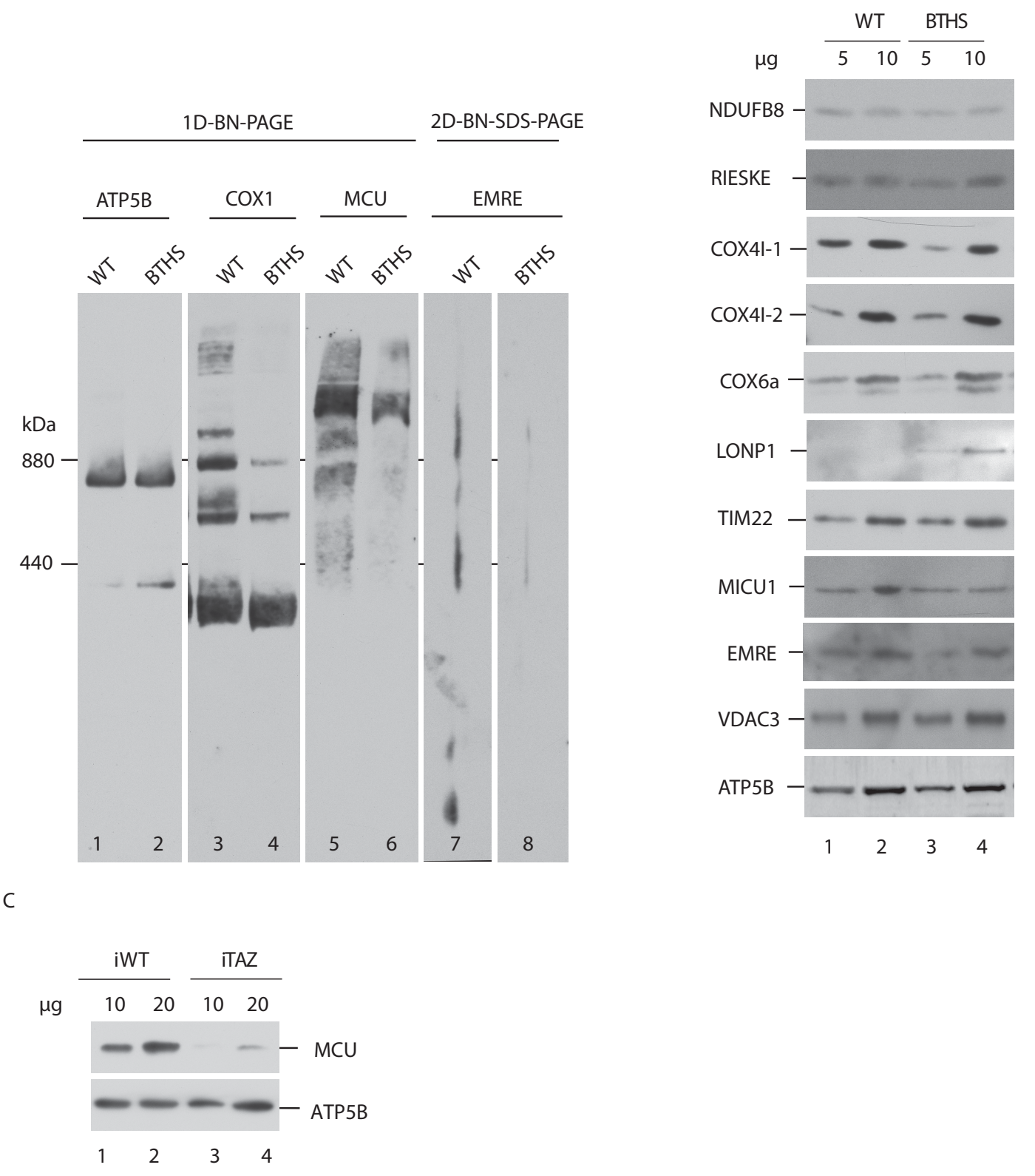

Figure 3.29: MCU protein steady state levels are reduced in BTHS mouse heart mitochondria

(A) Mitochondria from WT and BTHS mouse mitochondria were isolated by differential centrifugation and separated on a 12,5\% SDS-PAGE resolving gel. Indicated proteins were detected by immunoblotting.

(B) WT and BTHS mouse heart mitochodria were solubilized by $1 \%$ digitonin and separated on $\mathrm{BN}$ PAGE resolving gel (4-13\%). For detection of EMRE, single BN-PAGE lane was additionally separated in the second denaturing condition. Protein complexes were detected by immunoblotting against COX1, MCU, EMRE and ATP5B

(C) Steady state levels of MCU and ATP5B from BTHS patient-derived induced pluripotent stem cell cardiomyocytes (iPSC cardiomyocytes). Separation on 12,5\% SDS-PAGE and western blotting against MCU and ATP5B 


\section{Discussion}

\subsection{MCU complex organization in BTHS models}

The comparison of separated MCU complexes after 2D-BN-SDS-PAGE analysis of mouse organs displayed, that the migration patterns of MCU complexes differ in the observed organs (Figure 3.24). Liver, brain, skeletal muscle and heart are metabolically important organs, which differ in their involvement of calcium signaling in metabolic pathways and cellular processes (Paillard et al., 2017; Wescott et al., 2019). The direct comparison of MCU complex sizes among mouse organs showed that liver MCU complex distribution varies from the other organs in the size of the smallest detectable MCU complex and the complex organization (Figure 3.28). The predominant form of MCU in mouse liver is a $400 \mathrm{kDa}$ complex. In contrast, the smallest detectable form of MCU in mouse skeletal muscle, heart, brain and MEF cells is a $500 \mathrm{kDa}$ MCU complex (MCU-3). The reason for this size difference of MCU complexes between liver $(400 \mathrm{kDa})$ and of the other organs $(500 \mathrm{kDa})$ could not be clarified in this study. The presumable lack of one MCU component in liver MCU complex could cause the size difference between $500 \mathrm{kDa}$ and $400 \mathrm{kDa}$. The known MCU constituents (MCU, MCUR1, MCUb, MICU1/2) have a molecular weight ranging from 34 to $50 \mathrm{kDa}$. The lack of one of these components could explain the size shift. MCU protomers are organized as tetramers forming the calciumconducting pore (Baradaran et al., 2018; Fan et al., 2018; Oxenoid et al., 2016; Yoo et al., 2018). Therefore, two to four monomers of the known MCU interaction partners (MCUR1, MCUb, MICU1/2) would cover a size difference of $100 \mathrm{kDa}$ between a 400 and a $500 \mathrm{kDa}$ complex. For instance, it has been shown, that MCUb interacts with each MCU protomer in WT mammalian cells (Lambert et al., 2020). The same finding was made for MCU-EMRE interaction. Each MCU protomer of the MCU pore interacts with one single EMRE (Wang et al., 2019). The stoichiometry of MCU to other interaction partners can change from organ to organ (Paillard et al., 2017) and also depends on the physiological environment (Lambert et al., 2019). For instance, the MCU-to-MCUb ratio is relatively high in skeletal muscle and is reduced in cardiac tissues (Paillard et al., 2017). Another example of adapting MCU complex compositions is the MCUb-to-MCU ratio during cardiac failure. MCU and the 
gatekeepers MICU1 and MICU2 are displaced by MCUb in cardiac injury, to prevent calcium overload into cardiac mitochondria. This stress responsive mechanism has the consequence that the MCU complex size is lowered because of lacking gatekeepers, which can interact with MCU, but not with MCUb (Lambert et al., 2019).

Those considerations about adapting ratios of $\mathrm{MCU}$ to $\mathrm{MCU}$ interaction partners show that the MCU complex composition underlies a basic tetrameric structure, but the incorporation or release of MCU interaction partners go along with cellular and mitochondrial requirements. Our work confirms, that MCU-EMRE complex organization is tissue-specific.

\subsection{Increased basal mitochondrial calcium uptake and the role of MICU1 in TAZ KO MEF cells}

The investigation of MCU complexes in BTHS mouse brain, skeletal muscle and TAZ KO MEF cells showed, that the integrity of higher-order MCU complexes depends on cardiolipin (Figure 3.3). To understand the physiological relevance of altered MCU complex organization on the mitochondrial calcium homeostasis, the role of MCU gatekeepers MICU1 and MICU2 was addressed. The MCU constituents MICU1 and MICU2 play a crucial role in the regulation of MCU activity (Csordas et al., 2013; Mallilankaraman et al., 2012; Patron et al., 2014; Petrungaro et al., 2015). The loss of MCU gatekeeping function can result in mitochondrial calcium overload and mitochondrial permeability transition pore (MPTP) opening (Antony et al., 2016; Logan et al., 2014; Mallilankaraman et al., 2012; Patron et al., 2014). MCU-EMRE complexes of neuronal mitochondria, lacking MICU1/2 show increased mitochondrial calcium uptake and vulnerability to calcium overload leading to neuronal death (König et al., 2016). The results of this work show, that MCU complex organization in TAZ KO MEF cells and BTHS mouse brain and skeletal muscle is altered. The ratio of $\mathrm{MCU}$ complexes in the $\mathrm{MDa}$ range to $\mathrm{MCU}$ complexes in the $\mathrm{kDa}$ range is shifted to the $\mathrm{kDa}$ range in mitochondria from TAZ KO MEF cells and BTHS mouse skeletal muscle and brain (Figure 3.3, Figure 3.23, Figure 3.26). MCU complexes in MEF cells appear in three different molecular weight forms, called MCU-1 $(>2$ MDa), MCU-2 (1 MDa) and MCU-3 (500 kDa) (Figure 3.3). All MCU complexes consist of the integral membrane protein EMRE, an integral membrane protein, which 
interacts with the MCU pore-forming subunit and is essential to uptake cellular calcium into mitochondria of mammalian cells (Tsai et al., 2017). The gatekeeping subunit MICU1 was detected only in the MCU-1 complex by depletion of a MICU1containing MCU complex via coimmunprecipitation (Figure 3.7). The MCU-1 complex is absent in TAZ KO MEF cells, which might have physiological consequences for the cellular, or at least, the mitochondrial calcium homeostasis. Measurements of mitochondrial calcium uptake in WT and TAZ KO MEF cells displayed that the basal mitochondrial calcium uptake is increased in TAZ KO MEF mitochondria (Figure 3.12). The co-ocurrence of elevated basal mitochondrial calcium uptake and MICU1-lacking MCU complexes was observed in previous studies (König et al., 2016; Petrungaro et al., 2015). If the concentration of cellular calcium increases beyond a threshold concentration $(0,1-1 \mu \mathrm{M}$ approximately), binding of calcium to MICU subunits initiate the MCU pore opening and calcium conductance into mitochondria (Figure 4.1) (Kamer et al., 2017; Liu et al., 2016; Paillard et al., 2018). MICU1-lacking MCU complexes are constitutively active and mitochondrial calcium uptake is not regulated under these conditions (König et al., 2016; Petrungaro et al., 2015). MICU1-lacking MCU complexes in TAZ KO MEF cells could cause unregulated and increased basal mitochondrial calcium uptake in our study. This was considered as an initial working model, but missing information about the further composition of MCU complexes in MEF cells did not allow a statement on the causality between the MCU complex ratio shifts and the increased basal calcium levels in TAZ KO MEF cells. The identification of MCUb- and MCUR1-containing MCU complexes was not successful in this study. MCUb is discussed to inhibit MCU activity (Raffaello et al., 2013; Lambert et al., 2019; Paillard et al., 2017), whereas MCUR1 is a regulator of MCU required for mitochondrial calcium uptake (Adlakha et al., 2019; Mallilankaraman et al., 2012, 2015; Tomar et al., 2016; Vais et al., 2015). Both MCU subunits or additional unknown MCU components could cause the observed increased mitochondrial calcium levels in TAZ KO MEF cells.

Conclusively our hypothesis is, that the lack of tafazzin in BTHS mouse models causes the assembly and stability defect of MICU1-containing MCU complexes. Therefore, unregulated MCU-EMRE complexes are constitutively active and basal uptake of cytosolic calcium into mitochondria is increased. 


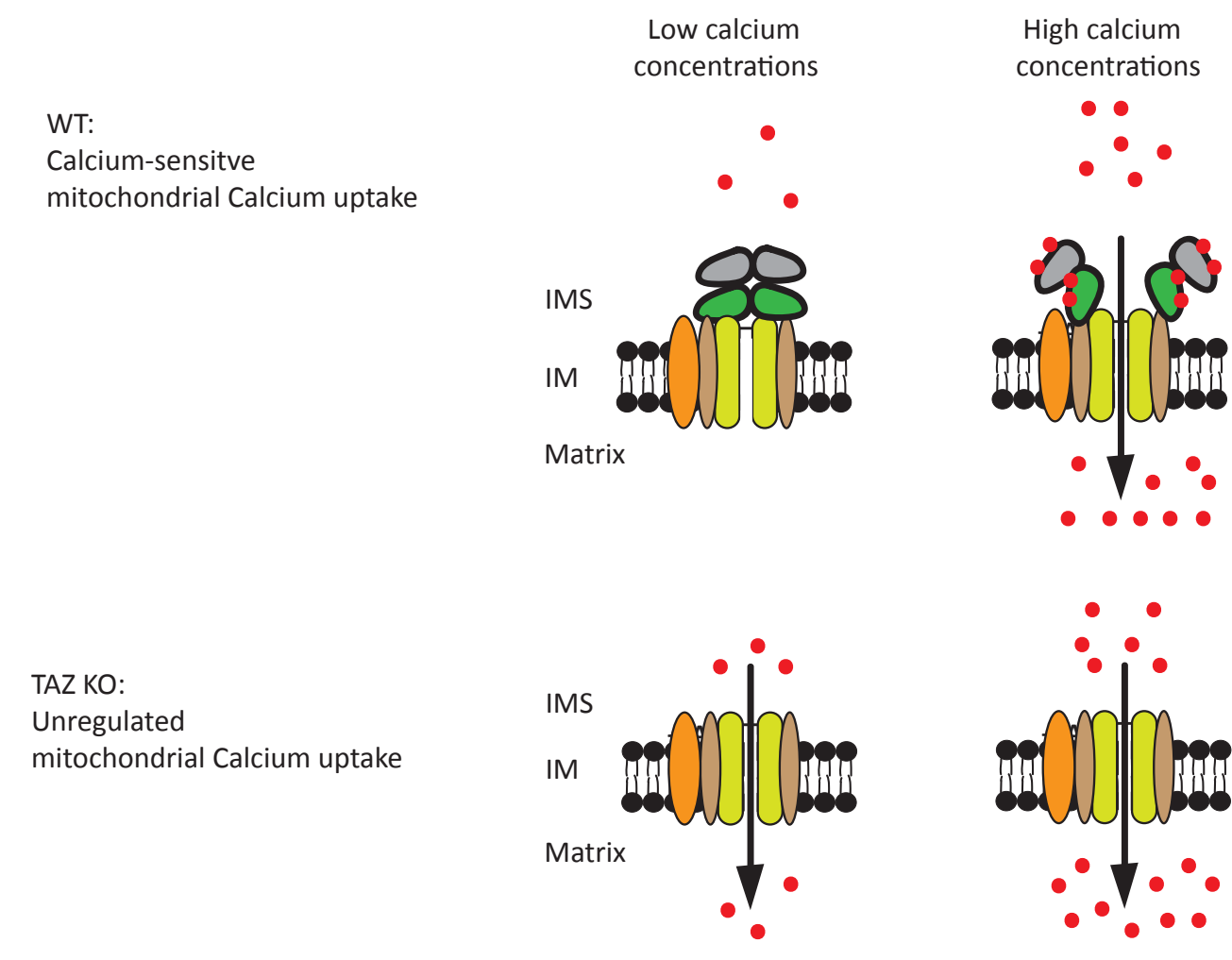

Figure 4.1: Proposed mitochondrial calcium uptake in WT and TAZ KO MEF cells

The concentration of cytosolic calcium increases beyond a threshold concentration and binding of calcium to MICU subunits can initiate calcium conductance into mitochondria via MCU pore opening. The model suggests, that less gatekeeping of MCU activity causes a constitutively active MCU-EMRE complex, which enables increased basal mitochondrial calcium uptake in TAZ KO MEF cells.

\subsection{MCU complex formation and role of reactive oxygen species (ROS)}

This work focused on how the lack of taffazin affects MCU complex organization. Protein-lipid interactions may maintain the structural integrity of MCU oligomers. In comparison to respiratory chain complexes (Sedlak and Robinson, 1999), stabilizing protein-cardiolipin interfaces in MCU are not known. MCU and respiratory chain complex integrity depend on cardiolipin remodeling (Figure 3.9). Putative MCUcardiolipin interfaces might affect the assembly and stability of MCU complexes. MCU complex assembly and organization could also be regulated by posttranslational modifications or the mitochondrial redox status (Dong et al., 2017). 
Calcium signaling and oxidative stress often act together to maintain cellular functions. The main source of ATP production is oxidative phosphorylation, but respiratory performance always holds the risk of increased reactive oxygen species (ROS) production. Production of mitochondrial ROS takes place in the respiratory chain and could cause cell damage, but is essential for regulatory functions as well (Brookes et al., 2004, Hamanaka and Chandel, 2010, Kiselyov and Muallem, 2016). Respiratory chain supercomplex formation (Figures 3.27, 3.30) and functions are impaired in Barth Syndrome models. Supercomplex formation is suggested to increase the efficiency of the electron transport within the respiratory chain and is proposed to reduce formation of ROS (Actin-Perez et al, 2008). ROS production is increased in BTHS mouse hearts and BTHS patient-derived induced pluripotent cardiomyocytes (iPSCs) (Dudek et al., 2016). It can be speculated, that ROS production and accumulation over life-time contributes to the pathophysiology of the BTHS mouse heart. The role of ROS damage and signaling in Barth Syndrome disease development remains still unclear. Beside the Barth Syndrome, it has been shown that increased ROS production could activate mitochondrial calcium uptake via MCU regulation (Dong et al., 2017). MCU undergoes several posttranslational modifications, which could affect its activity and its oligomerization state. It has been observed, that the N-terminal domain of MCU (MCU-NTD) contains a conserved cysteine (Cys-97), which could even act as a redox sensor. Oxidized Cys-97 undergoes S-glutathionylation upon oxidative stress. Fast protein liquid chromatography (FLPC) analysis showed, that oxidation or mutation of Cys-97 could promote oligomerization of a 450-600 kDa MCU complex in HEK and Hela cells. Modification of Cys-97 revealed higher mitochondrial calcium uptake through MCU pore (Dong et al., 2017). The human 450-600 kDa MCU complex resolved by FLPC analysis reminds of the $400 / 500 \mathrm{kDa}$ MCU complexes in mouse organs and MEF cells (MCU-3, Figure 3.2, 3.24). This complex may be the smallest member of MCU complex oligomers allowing regulated mitochondrial calcium uptake in mammalian cells. The observation of redox-dependent MCU oligomerization via FLPC analysis by Dong et al., 2017 initiated to address the role of ROS in MCU complex organization by BN-PAGE analysis (Figure 3.11). ROS quenching by Mitotempo could reduce the elevated ROS levels in TAZ KO MEF cells, but the reduction of mitochondrial ROS production could not show alterations in MCU oligomerization after 2-BN-SDS-PAGE analysis. Beside redox-dependent regulation of the MCU 
protein, it was quite obvious that the redox status of the intermembrane space could affect the redox-dependent MICU1 and MICU2 dimerization (Petrungaro et al., 2015). Shifts in the redox state of the MICU gatekeepers from WT to TAZ KO MEF cells should also affect the MCU complex organization. Figure 3.1 showed, that the protein steady state levels of reduced MICU1 and MICU2 (total amounts) are not altered in TAZ KO. MICU1 and MICU2 protein levels on a non-reducing SDS-PAGE were also examined, but have not shown any differences in the levels of MICU monomers (reduced form) to MICU dimers (oxidized form) (Data not shown). Thus, a redox ratio shift of MICU1 and MICU2 has not been observed, which would affect MCU complex assembly in TAZ KO MEF mitochondria.

Conclusively, this work has not shown an effect of ROS on MCU complex organization.

\subsection{Role of cardiolipin on MCU and respiratory chain complexes in cardiac tissues}

$90 \%$ of metabolic energy in cardiomyocytes are provided by the mitochondrial respiratory chain. Fatty acids are the main carbon source under physiological conditions, which explains the high oxygen demand enabling fatty acid catabolism (Grynberg and Demaison, 1996). Cardiac fatty acid catabolism is coupled to the further oxidation of reducing equivalents the respiratory chain. Respiratory chain complexes are organized to supercomplexes/respirasomes composed of complex I, complex III and complex IV in mammalian cells (Althoff et al., 2011; Schägger and Pfeiffer, 2000). Cardiolipin enables the stabilization of every single respiratory chain complex and is required for their enzymatic activity. Additionally, stabilization and function of respiratory chain supercomplexes are dependent on cardiolipin (Bazan et al., 2012; Eble et al., 1990; Sharpley et al., 2006). Thus, defect in cardiolipin maturation in cardiomyocytes affects respiratory chain activity and stability of complexes. The Barth Syndrome (BTHS) is caused by impaired remodeling of cardiolipin by its acyltranferase tafazzin. The tafazzin deficiency in BTHS models displays different organ-specific phenotypes. Respiratory chain and MCU complexes in isolated mitochondria from BTHS mouse livers do not show differences in the organization of the observed oligomeric states of mitochondrial protein complexes 
(Figure 3.28, Dudek et al., 2016). Instead, cardiac respiratory chain supercomplexes are dissociated and respiratory chain is remodeled (Figure 3.30, Dudek et al., 2016). This study reveals that MCU protein steady state levels in BTHS mouse hearts are significantly reduced (Figure 3.29). MCU and EMRE containing complexes in BTHS mouse hearts are reduced, but they are not differently organized or display a ratio shift of higher to lower molecular weight forms from WT to BTHS (Figure 3.30). Instead, MCU complexes from TAZ KO MEF cells, BTHS mouse brain and skeletal muscle show the phenotype of dissociated MDa MCU complexes and a reorganized distribution of the MCU protein from MDa to $\mathrm{kDa} M C U$ complexes. The cardiac specificity of remodeled respiratory chain in comparison to kidney and liver and the reduction of MCU complexes in BTHS mouse hearts were explained by a cardiacspecific cardiolipin species (Dudek et al., 2018). The predominant cardiolipin (CL) species in the heart is tetra-linoleoyl-CL (CL 72:8). Lack of tafazzin causes an increase of the precursor monolysocardiolipin (MLCL) in all mouse tissues, but the cardiolipin pools in various mouse organs are different. It is still unknown in general, which cardiolipin species promote protein complex stability or how cardiolipin species are distributed over respirasomes, translocases, and other mitochondrial protein complexes. In addition, it is not documented, how accumulated MLCL in BTHS models affect protein complex stability. However, the symptoms of the human BTHS disease show that cardiolipin deficiency is more deleterious in cardiac tissues as in other organs. For instance, the heart contains cardiac-specific isoforms of respiratory chain constituents (Grossman and Lomax, 1997). COX4a is a cardiacspecific and cardiolipin-dependent subunit of complex VI and applies regulatory functions (Dudek et al., 2016). Perhaps, the cardiac tissue-specificity of cardiolipin species could explain the drastic reduction of MCU protein steady state levels in BTHS mouse hearts (Figure 3.29). Complex II deficiency was observed in BTHS mouse hearts and BTHS patient-derived iPSC cardiomyocytes (Dudek et al., 2016). The absence of protein complexes in cardiac BTHS mouse mitochondria could refer to an import defect of one of its subunits or an increased turnover of one of the complex constituents. The following chapter focuses on these two possibilities to explain the absence of protein complexes in BTHS mouse hearts. 


\subsection{Mitochondrial import and stability of MCU precursor protein}

MCU and SDHA steady state levels in mitochondria from BTHS mouse hearts are reduced (Figure 3.28; Dudek et al., 2016). The reduction of both mitochondrial proteins might underlie the same cardiolipin-dependent mechanism. The precursor import of selected mitochondrial proteins could be affected in BTHS mouse hearts. In-vitro preprotein imports of MCU and the complex II subunit SDHA into purified mouse heart mitochondria could not be established. The in-vitro import of MCU was established in purified WT and TAZ KO MEF mitochondria (Figure 3.14). The invitro import of MCU preprotein into MEF TAZ KO MEF mitochondria displays reduced mature MCU (Figure 3.17), whereas the MCU protein steady state levels are unaffected in TAZ KO MEF cells (Figure 3.1). In fact, the precursor import machinery (Figure 3.13) and the in-vitro import of the mitochondrial control preproteins EMRE and OTC are unaffected in TAZ KO MEF mitochondria (Figure 3.15). In-vitro import of MCU preprotein into purified CCCP-treated mitochondria displays that the MCU import is more sensitive to reduced membrane potential than the import of the control precursor OTC (Figure 3.22). The impaired import of MCU preprotein into MEF TAZ KO mitochondria and the reduced MCU levels in BTHS mouse hearts might underlie the same taffazin-dependent mechanism. In this scenario, TAZ KO MEF cells are capable to compensate the impaired MCU import and MCU protein steady state levels are not reduced. Instead, reduced MCU levels in BTHS mouse heart mitochondria are not compensated. The second speculation for reduced MCU in BTHS mouse hearts is accelerated turnover of the MCU protein. Turnover assays of endogenous MCU in iWT and iTAZ iPSC cardiomyocytes have not clarified yet, if newly translated MCU degrades faster in iTAZ iPSC cardiomyocytes than in WT iPSC cardiomyocytes. The pulse-chase assay of newly imported MCU precursor into purified WT and TAZ KO MEF mitochondria indicates, that MCU does not degrade faster in mitochondria lacking taffazin (Figure 3.20). In general, the MCU protein is more stable, whereas EMRE degrades within 4 hours after inhibition of cytosolic translation (Tsai et al., 2017). The half-life of the MCU protein is more than 24 hours, whereas MICU1 has a half-life of approximately 5 hours (Petrungaro et al., 2015). 
Conclusively, in-vitro import of MCU precursors into TAZ KO MEF mitochondria displays reduced mature $\mathrm{MCU}$, but not an increased turnover rate of newly imported MCU. It is speculative, if these findings in TAZ KO MEF cells can explain the observed phenotype of reduced MCU in BTHS mouse hearts.

\section{Conclusion of results}

This work has shown that WT MEF cells contain 3 different molecular weight forms of MCU, which undergo a complex ratio shift from WT to TAZ KO MEF mitochondria (Figure 3.4). The MCU complex ratio shift is specifically dependent on the phospholipid composition of mitochondrial membranes and not on regulation by ROS or other stimuli (Figure 3.11). The tafazzin complementation of TAZ KO MEF cells has shown that remodeled cardiolipin is required for the structural integrity of MCU complexes in the MDa range, but MCU oligomers with a size of 400-500 kDa do not require sufficient cardiolipin synthesis and remodeling (Figure 3.11). The MCU protein is distributed approximately $60 \%$ to the $1000 \mathrm{kDa}$ (MCU-2) and $40 \%$ to the $500 \mathrm{kDa}(\mathrm{MCU}-3)$ form, whereas $90 \%$ of the MCU protein is distributed to the $500 \mathrm{kDa}$ form in TAZ KO MEF cells (Figure 3.12). The composition of those MCU complexes could not be solved completely in this study. The MICU gatekeepers reside in the higher MDa MCU complex (Figure 3.7), whereas information about the published MCU interaction partners MCUb and MCUR1 could not be provided. However, EMRE always interacts with the MCU pore-forming subunit, no matter, how the integral module of the uniporter is shifted between the MCU complexes, dependent on the phospholipid environment. Thus, the interaction of the pore-forming subunit MCU and its regulator EMRE is not affected in TAZ KO MEF mitochondria (Figure 3.8). In addition, the interaction of newly in-vitro imported MCU with endogenous EMRE is not affected in TAZ KO mitochondria (Figure 3.19). The same finding has been observed by in-vitro import of EMRE with exogenous, FLAGtagged MCU (Figure 3.17). Imported EMRE assembles with MCU and forms the two $\mathrm{kDa} \mathrm{MCU}$ complexes with the characteristic complex ratio pattern in WT and TAZ KO MEF cells. The TIM23 complex-dependent protein import machinery is not affected in TAZ KO MEF cells. The protein steady state levels of core translocase 
constituents (Tim23, Tom and Tim22 complex) are not altered in TAZ KO MEF cells as well as the structural integrity of translocase complexes (Figure 3.14). The in-vitro import of the preproteins EMRE and the motor-dependent, matrix-targeted OTC are not affected in isolated TAZ KO mitochondria (Figure 3.16), whereas the mature form of in-vitro imported MCU in TAZ KO is reduced (Figure 3.18). The paradox is, that the protein steady state levels of MCU, EMRE and other uniporter constituents are not affected in TAZ KO MEF cells (Figure 3.1), whereas the MCU import seems to show a reduction (Figure 3.18). The reduced amount of matured MCU after in-vitro import cannot be explained by an increased turnover of newly imported MCU (Figure 3.20). The insertion of 2-TMD-containing proteins into the inner mitochondrial membrane in general (Figure 3.21) and the lack of one constituent on the MCU pore in TAZ KO (MICU1) cannot explain the reduced amount of mature MCU (Figure 3.22). However, MCU imports into CCCP-titrated WT mitochondria showed, that MCU imports are highly membrane potential-sensitive in comparison to the model precursor import of OTC (Figure 3.23). The reduced MCU import into TAZ KO mitochondria does not fit to the unaltered MCU steady state levels in WT and TAZ KO MEF cells. MCU protein steady state levels in BTHS mouse heart mitochondria are reduced instead (Figure 3.29). This phenotype is not observed in BTHS mouse liver or brain (Figure 3.29). MCU complexes in cardiac BTHS mouse mitochondria are reduced, but do not display a changed complex organization in tafazzin-lacking mitochondria (Figure 3.30). MCU complexes in BTHS mouse brain and skeletal muscle undergo a MCU complex ratio shift from WT to BTHS, comparable to WT and TAZ KO MEF cells (Figure 3.26).

\section{Future perspectives}

\subsection{MCU reduction in BTHS cardiac mitochondria}

The reduction of MCU protein levels in BTHS mouse hearts could be caused by an increased turnover of newly translated $\mathrm{MCU}$ protein or an import defect into mitochondria. The decreased levels of MCU are also found in BTHS patient-derived induced pluripotent stem cell (iPSC) cardiomyocytes. To study the turnover of MCU 
protein in iWT and iTAZ cardiomyocytes, they can be treated with emetine, which inhibits cytosolic translation. iPSC cardiomyocytes are a suitable model to study mitochondrial calcium uptake by ratiometric calcium sensors, which is difficult to perform with isolated mitochondria from mouse tissues. It would be interesting to investigate, if cardiac BTHS mitochondria show decreased mitochondrial calcium uptake.

\subsection{MCU interaction partners and complex composition}

The MCU complex composition of the MCU-2 and MCU-3 in MEF cells could not be fully clarified. The putative inhibitory function of MCUb could affect calcium uptake into mitochondria. Downregulation of MCUb and MCUR1 via RNAi would give more information about MCUb- and MCUR1-containing MCU complexes on 2DBN-SDS-PAGE. MCUb and MCUR1 antibodies will be tested for native Co-IP to investigate both, the interaction with other MCU constituents in TAZ KO and the determination of MCUb/MCUR1-containing MCU complexes by depletion coimmunprecipitation. The importance of this question was explained by several findings of the last years, that the MCU complex composition and presence/absence of MCU components always determine the uniporter activity directly. Altered mitochondrial calcium homeostasis in BTHS could affect putative calcium-dependent processes, such as apoptotic signaling, Krebs cycle regulation and respiratory performance.

\section{Bibliography}

Acehan, D., Vaz, F., Houtkooper, R.H., James, J., Moore, V., Tokunaga, C., Kulik, W., Wansapura, J., Toth, M.J., Strauss, A., et al. (2011). Cardiac and skeletal muscle defects in a mouse model of human Barth syndrome. J. Biol. Chem. 286, 899-908.

Acín-Pérez, R., Fernández-Silva, P., Peleato, M.L., Pérez-Martos, A., and Enriquez, J.A. (2008). Respiratory active mitochondrial supercomplexes. Mol. Cell 32, 529539.

Adlakha, J., Karamichali, I., Sangwallek, J., Deiss, S., Bär, K., Coles, M., Hartmann, M.D., Lupas, A.N., and Hernandez Alvarez, B. (2019). Characterization of MCU- 
Binding Proteins MCUR1 and CCDC90B - Representatives of a Protein Family Conserved in Prokaryotes and Eukaryotic Organelles. Struct. Lond. Engl. 1993 27, 464-475.e6.

Althoff, T., Mills, D.J., Popot, J.-L., and Kühlbrandt, W. (2011). Arrangement of electron transport chain components in bovine mitochondrial supercomplex I1III2IV1. EMBO J. 30, 4652-4664.

Antón, Z., Landajuela, A., Hervás, J.H., Montes, L.R., Hernández-Tiedra, S., Velasco, G., Goñi, F.M., and Alonso, A. (2016). Human Atg8-cardiolipin interactions in mitophagy: Specific properties of LC3B, GABARAPL2 and GABARAP. Autophagy 12, 2386-2403.

Antony, A.N., Paillard, M., Moffat, C., Juskeviciute, E., Correnti, J., Bolon, B., Rubin, E., Csordás, G., Seifert, E.L., Hoek, J.B., et al. (2016). MICU1 regulation of mitochondrial $\mathrm{Ca}(2+)$ uptake dictates survival and tissue regeneration. Nat. Commun. $7,10955$.

Baradaran, R., Wang, C., Siliciano, A.F., and Long, S.B. (2018). Cryo-EM structures of fungal and metazoan mitochondrial calcium uniporters. Nature 559, 580-584.

Barth, P.G., Scholte, H.R., Berden, J.A., Van der Klei-Van Moorsel, J.M., LuytHouwen, I.E., Van 't Veer-Korthof, E.T., Van der Harten, J.J., and Sobotka-Plojhar, M.A. (1983). An X-linked mitochondrial disease affecting cardiac muscle, skeletal muscle and neutrophil leucocytes. J. Neurol. Sci. 62, 327-355.

Barth, P.G., Wanders, R.J., Vreken, P., Janssen, E.A., Lam, J., and Baas, F. (1999). $\mathrm{X}$-linked cardioskeletal myopathy and neutropenia (Barth syndrome) (MIM 302060). J. Inherit. Metab. Dis. 22, 555-567.

Baughman, J.M., Perocchi, F., Girgis, H.S., Plovanich, M., Belcher-Timme, C.A., Sancak, Y., Bao, X.R., Strittmatter, L., Goldberger, O., Bogorad, R.L., et al. (2011). Integrative genomics identifies MCU as an essential component of the mitochondrial calcium uniporter. Nature 476, 341-345.

Bazán, S., Mileykovskaya, E., Mallampalli, V.K.P.S., Heacock, P., Sparagna, G.C., and Dowhan, W. (2013). Cardiolipin-dependent reconstitution of respiratory supercomplexes from purified Saccharomyces cerevisiae complexes III and IV. J. Biol. Chem. 288, 401-411.

Belosludtsev, K.N., Dubinin, M.V., Belosludtseva, N.V., and Mironova, G.D. (2019). Mitochondrial Ca2+ Transport: Mechanisms, Molecular Structures, and Role in Cells. Biochem. Biokhimiia 84, 593-607.

Bers, D.M., and Weber, C.R. (2002). Na/Ca exchange function in intact ventricular myocytes. Ann. N. Y. Acad. Sci. 976, 500-512.

Bertero, E., and Maack, C. (2018). Calcium Signaling and Reactive Oxygen Species in Mitochondria. Circ. Res. 122, 1460-1478. 
Bione, S., D’Adamo, P., Maestrini, E., Gedeon, A.K., Bolhuis, P.A., and Toniolo, D. (1996). A novel X-linked gene, G4.5. is responsible for Barth syndrome. Nat. Genet. $12,385-389$.

Bissler, J.J., Tsoras, M., Göring, H.H.H., Hug, P., Chuck, G., Tombragel, E., McGraw, C., Schlotman, J., Ralston, M.A., and Hug, G. (2002). Infantile dilated Xlinked cardiomyopathy, G4.5 mutations, altered lipids, and ultrastructural malformations of mitochondria in heart, liver, and skeletal muscle. Lab. Investig. J. Tech. Methods Pathol. 82, 335-344.

Boynton, T.O., and Shimkets, L.J. (2015). Myxococcus CsgA, Drosophila Sniffer, and human HSD10 are cardiolipin phospholipases. Genes Dev. 29, 1903-1914.

Brandner, K., Mick, D.U., Frazier, A.E., Taylor, R.D., Meisinger, C., and Rehling, P. (2005). Taz1, an outer mitochondrial membrane protein, affects stability and assembly of inner membrane protein complexes: implications for Barth Syndrome. Mol. Biol. Cell 16, 5202-5214.

Buckland, A.G., Kinkaid, A.R., and Wilton, D.C. (1998). Cardiolipin hydrolysis by human phospholipases A2. The multiple enzymatic activities of human cytosolic phospholipase A2. Biochim. Biophys. Acta 1390, 65-72.

Bunse, M., Bit-Avragim, N., Riefflin, A., Perrot, A., Schmidt, O., Kreuz, F.R., Dietz, R., Jung, W.-I., and Osterziel, K.J. (2003). Cardiac energetics correlates to myocardial hypertrophy in Friedreich's ataxia. Ann. Neurol. 53, 121-123.

Chacinska, A., Koehler, C.M., Milenkovic, D., Lithgow, T., and Pfanner, N. (2009). Importing mitochondrial proteins: machineries and mechanisms. Cell 138, 628-644.

Chang, S.C., Heacock, P.N., Mileykovskaya, E., Voelker, D.R., and Dowhan, W. (1998). Isolation and characterization of the gene (CLS1) encoding cardiolipin synthase in Saccharomyces cerevisiae. J. Biol. Chem. 273, 14933-14941.

Chaudhuri, D., Artiga, D.J., Abiria, S.A., and Clapham, D.E. (2016). Mitochondrial calcium uniporter regulator 1 (MCUR1) regulates the calcium threshold for the mitochondrial permeability transition. Proc. Natl. Acad. Sci. U. S. A. 113, E18721880 .

Checchetto, V., and Szabò, I. (2019). MCU Regulation in Lipid Bilayer and Electrophysiological Recording. Methods Mol. Biol. Clifton NJ 1925, 59-63.

Chen, D., Zhang, X.-Y., and Shi, Y. (2006). Identification and functional characterization of hCLS1, a human cardiolipin synthase localized in mitochondria. Biochem. J. 398, 169-176.

Chowdhury, A., Aich, A., Jain, G., Wozny, K., Lüchtenborg, C., Hartmann, M., Bernhard, O., Balleiniger, M., Alfar, E.A., Zieseniss, A., et al. (2018). Defective Mitochondrial Cardiolipin Remodeling Dampens HIF-1 $\alpha$ Expression in Hypoxia. Cell Rep. 25, 561-570.e6.

Chu, C.T., Ji, J., Dagda, R.K., Jiang, J.F., Tyurina, Y.Y., Kapralov, A.A., Tyurin, V.A., Yanamala, N., Shrivastava, I.H., Mohammadyani, D., et al. (2013). Cardiolipin 
externalization to the outer mitochondrial membrane acts as an elimination signal for mitophagy in neuronal cells. Nat. Cell Biol. 15, 1197-1205.

Clarke, S.L.N., Bowron, A., Gonzalez, I.L., Groves, S.J., Newbury-Ecob, R., Clayton, N., Martin, R.P., Tsai-Goodman, B., Garratt, V., Ashworth, M., et al. (2013). Barth syndrome. Orphanet J. Rare Dis. 8, 23.

Claypool, S.M., and Koehler, C.M. (2012). The complexity of cardiolipin in health and disease. Trends Biochem. Sci. 37, 32-41.

Claypool, S.M., McCaffery, J.M., and Koehler, C.M. (2006). Mitochondrial mislocalization and altered assembly of a cluster of Barth syndrome mutant tafazzins. J. Cell Biol. 174, 379-390.

Cole, L.K., Mejia, E.M., Vandel, M., Sparagna, G.C., Claypool, S.M., Dyck-Chan, L., Klein, J., and Hatch, G.M. (2016). Impaired Cardiolipin Biosynthesis Prevents Hepatic Steatosis and Diet-Induced Obesity. Diabetes 65, 3289-3300.

Colina-Tenorio, L., Horten, P., Pfanner, N., and Rampelt, H. (2020). Shaping the mitochondrial inner membrane in health and disease. J. Intern. Med.

Connerth, M., Tatsuta, T., Haag, M., Klecker, T., Westermann, B., and Langer, T. (2012). Intramitochondrial transport of phosphatidic acid in yeast by a lipid transfer protein. Science 338, 815-818.

Cosson, L., Toutain, A., Simard, G., Kulik, W., Matyas, G., Guichet, A., Blasco, H., Maakaroun-Vermesse, Z., Vaillant, M.-C., Le Caignec, C., et al. (2012). Barth syndrome in a female patient. Mol. Genet. Metab. 106, 115-120.

Csordás, G., Golenár, T., Seifert, E.L., Kamer, K.J., Sancak, Y., Perocchi, F., Moffat, C., Weaver, D., de la Fuente Perez, S., Bogorad, R., et al. (2013). MICU1 controls both the threshold and cooperative activation of the mitochondrial $\mathrm{Ca}^{2+}$ uniporter. Cell Metab. 17, 976-987.

De Stefani, D., Raffaello, A., Teardo, E., Szabò, I., and Rizzuto, R. (2011a). A fortykilodalton protein of the inner membrane is the mitochondrial calcium uniporter. Nature 476, 336-340.

De Stefani, D., Raffaello, A., Teardo, E., Szabò, I., and Rizzuto, R. (2011b). A fortykilodalton protein of the inner membrane is the mitochondrial calcium uniporter. Nature 476, 336-340.

Dudek, J., Cheng, I.-F., Balleininger, M., Vaz, F.M., Streckfuss-Bömeke, K., Hübscher, D., Vukotic, M., Wanders, R.J.A., Rehling, P., and Guan, K. (2013). Cardiolipin deficiency affects respiratory chain function and organization in an induced pluripotent stem cell model of Barth syndrome. Stem Cell Res. 11, 806-819.

Dudek, J., Cheng, I.-F., Chowdhury, A., Wozny, K., Balleininger, M., Reinhold, R., Grunau, S., Callegari, S., Toischer, K., Wanders, R.J., et al. (2016). Cardiac-specific succinate dehydrogenase deficiency in Barth syndrome. EMBO Mol. Med. 8, 139154. 
Dudek, J., Hartmann, M., and Rehling, P. (2019). The role of mitochondrial cardiolipin in heart function and its implication in cardiac disease. Biochim. Biophys. Acta Mol. Basis Dis. 1865, 810-821.

Eble, K.S., Coleman, W.B., Hantgan, R.R., and Cunningham, C.C. (1990). Tightly associated cardiolipin in the bovine heart mitochondrial ATP synthase as analyzed by 31P nuclear magnetic resonance spectroscopy. J. Biol. Chem. 265, 19434-19440.

Elbaz-Alon, Y., Rosenfeld-Gur, E., Shinder, V., Futerman, A.H., Geiger, T., and Schuldiner, M. (2014). A dynamic interface between vacuoles and mitochondria in yeast. Dev. Cell 30, 95-102.

Eshima, H., Tamura, Y., Kakehi, S., Nakamura, K., Kurebayashi, N., Murayama, T., Kakigi, R., Sakurai, T., Kawamori, R., and Watada, H. (2019). Dysfunction of muscle contraction with impaired intracellular $\mathrm{Ca} 2+$ handling in skeletal muscle and the effect of exercise training in male $\mathrm{db} / \mathrm{db}$ mice. J. Appl. Physiol. Bethesda Md 1985 $126,170-182$.

Fan, C., Fan, M., Orlando, B.J., Fastman, N.M., Zhang, J., Xu, Y., Chambers, M.G., Xu, X., Perry, K., Liao, M., et al. (2018). X-ray and cryo-EM structures of the mitochondrial calcium uniporter. Nature 559, 575-579.

Ferri, L., Dionisi-Vici, C., Taurisano, R., Vaz, F.M., Guerrini, R., and Morrone, A. (2016). When silence is noise: infantile-onset Barth syndrome caused by a synonymous substitution affecting TAZ gene transcription. Clin. Genet. 90, 461-465.

Fiedorczuk, K., Letts, J.A., Degliesposti, G., Kaszuba, K., Skehel, M., and Sazanov, L.A. (2016). Atomic structure of the entire mammalian mitochondrial complex I. Nature 538, 406-410.

Fieni, F., Lee, S.B., Jan, Y.N., and Kirichok, Y. (2012). Activity of the mitochondrial calcium uniporter varies greatly between tissues. Nat. Commun. 3, 1317.

Friedman, J.R., Lackner, L.L., West, M., DiBenedetto, J.R., Nunnari, J., and Voeltz, G.K. (2011). ER Tubules Mark Sites of Mitochondrial Division. Science 334, 358362.

Gebert, N., Joshi, A.S., Kutik, S., Becker, T., McKenzie, M., Guan, X.L., Mooga, V.P., Stroud, D.A., Kulkarni, G., Wenk, M.R., et al. (2009). Mitochondrial cardiolipin involved in outer-membrane protein biogenesis: implications for Barth syndrome. Curr. Biol. CB 19, 2133-2139.

Geissler, A., Chacinska, A., Truscott, K.N., Wiedemann, N., Brandner, K., Sickmann, A., Meyer, H.E., Meisinger, C., Pfanner, N., and Rehling, P. (2002). The mitochondrial presequence translocase: an essential role of Tim50 in directing preproteins to the import channel. Cell 111, 507-518.

Giorgi, C., Danese, A., Missiroli, S., Patergnani, S., and Pinton, P. (2018). Calcium Dynamics as a Machine for Decoding Signals. Trends Cell Biol. 28, 258-273.

Gonzalvez, F., D’Aurelio, M., Boutant, M., Moustapha, A., Puech, J.-P., Landes, T., Arnauné-Pelloquin, L., Vial, G., Taleux, N., Slomianny, C., et al. (2013). Barth 
syndrome: cellular compensation of mitochondrial dysfunction and apoptosis inhibition due to changes in cardiolipin remodeling linked to tafazzin (TAZ) gene mutation. Biochim. Biophys. Acta 1832, 1194-1206.

Grossman, L.I., and Lomax, M.I. (1997). Nuclear genes for cytochrome c oxidase. Biochim. Biophys. Acta 1352, 174-192.

Grynberg, A., and Demaison, L. (1996). Fatty acid oxidation in the heart. J. Cardiovasc. Pharmacol. 28 Suppl 1, S11-17.

Hamanaka, R.B., and Chandel, N.S. (2010). Mitochondrial reactive oxygen species regulate cellular signaling and dictate biological outcomes. Trends Biochem. Sci. 35, 505-513.

Hatch, G.M., and McClarty, G. (2004). C. trachomatis-infection accelerates metabolism of phosphatidylcholine derived from low density lipoprotein but does not affect phosphatidylcholine secretion from hepatocytes. BMC Microbiol. 4, 8 .

von Heijne, G. (1986). Mitochondrial targeting sequences may form amphiphilic helices. EMBO J. 5, 1335-1342.

von Heijne, G. (1989). Control of topology and mode of assembly of a polytopic membrane protein by positively charged residues. Nature $341,456-458$.

Hoch, F.L. (1992a). Cardiolipins and biomembrane function. Biochim. Biophys. Acta $1113,71-133$.

Hoch, F.L. (1992b). Cardiolipins and biomembrane function. Biochim. Biophys. Acta $1113,71-133$.

Horvath, S.E., and Daum, G. (2013). Lipids of mitochondria. Prog. Lipid Res. 52, 590-614.

Hostetler, K.Y., Van den Bosch, H., and Van Deenen, L.L. (1971). Biosynthesis of cardiolipin in liver mitochondria. Biochim. Biophys. Acta 239, 113-119.

Houtkooper, R.H., Rodenburg, R.J., Thiels, C., van Lenthe, H., Stet, F., Poll-The, B.T., Stone, J.E., Steward, C.G., Wanders, R.J., Smeitink, J., et al. (2009a). Cardiolipin and monolysocardiolipin analysis in fibroblasts, lymphocytes, and tissues using high-performance liquid chromatography-mass spectrometry as a diagnostic test for Barth syndrome. Anal. Biochem. 387, 230-237.

Houtkooper, R.H., Turkenburg, M., Poll-The, B.T., Karall, D., Pérez-Cerdá, C., Morrone, A., Malvagia, S., Wanders, R.J., Kulik, W., and Vaz, F.M. (2009b). The enigmatic role of tafazzin in cardiolipin metabolism. Biochim. Biophys. Acta 1788, 2003-2014.

Hovius, R., Lambrechts, H., Nicolay, K., and de Kruijff, B. (1990). Improved methods to isolate and subfractionate rat liver mitochondria. Lipid composition of the inner and outer membrane. Biochim. Biophys. Acta 1021, 217-226. 
Hovius, R., Thijssen, J., van der Linden, P., Nicolay, K., and de Kruijff, B. (1993). Phospholipid asymmetry of the outer membrane of rat liver mitochondria. Evidence for the presence of cardiolipin on the outside of the outer membrane. FEBS Lett. 330, $71-76$.

Huang, H., Gao, Q., Peng, X., Choi, S.-Y., Sarma, K., Ren, H., Morris, A.J., and Frohman, M.A. (2011). piRNA-associated germline nuage formation and spermatogenesis require MitoPLD profusogenic mitochondrial-surface lipid signaling. Dev. Cell 20, 376-387.

Hurst, S., Baggett, A., Csordas, G., and Sheu, S.-S. (2019). SPG7 targets the m-AAA protease complex to process MCU for uniporter assembly, $\mathrm{Ca} 2+$ influx, and regulation of mitochondrial permeability transition pore opening. J. Biol. Chem. 294, $10807-10818$.

Kagan, V.E., Chu, C.T., Tyurina, Y.Y., Cheikhi, A., and Bayir, H. (2014). Cardiolipin asymmetry, oxidation and signaling. Chem. Phys. Lipids 179, 64-69.

Kamer, K.J., Grabarek, Z., and Mootha, V.K. (2017). High-affinity cooperative Ca2+ binding by MICU1-MICU2 serves as an on-off switch for the uniporter. EMBO Rep. $18,1397-1411$.

Kamer, K.J., Sancak, Y., Fomina, Y., Meisel, J.D., Chaudhuri, D., Grabarek, Z., and Mootha, V.K. (2018). MICU1 imparts the mitochondrial uniporter with the ability to discriminate between $\mathrm{Ca} 2+$ and $\mathrm{Mn} 2+$. Proc. Natl. Acad. Sci. U. S. A. 115, E7960E7969.

Kirichok, Y., Krapivinsky, G., and Clapham, D.E. (2004). The mitochondrial calcium uniporter is a highly selective ion channel. Nature 427, 360-364.

Kiselyov, K., and Muallem, S. (2016). ROS and intracellular ion channels. Cell Calcium 60, 108-114.

König, T., Tröder, S.E., Bakka, K., Korwitz, A., Richter-Dennerlein, R., Lampe, P.A., Patron, M., Mühlmeister, M., Guerrero-Castillo, S., Brandt, U., et al. (2016). The mAAA Protease Associated with Neurodegeneration Limits MCU Activity in Mitochondria. Mol. Cell 64, 148-162.

Kovács-Bogdán, E., Sancak, Y., Kamer, K.J., Plovanich, M., Jambhekar, A., Huber, R.J., Myre, M.A., Blower, M.D., and Mootha, V.K. (2014). Reconstitution of the mitochondrial calcium uniporter in yeast. Proc. Natl. Acad. Sci. U. S. A. 111, 89858990.

Koval, O.M., Nguyen, E.K., Santhana, V., Fidler, T.P., Sebag, S.C., Rasmussen, T.P., Mittauer, D.J., Strack, S., Goswami, P.C., Abel, E.D., et al. (2019). Loss of MCU prevents mitochondrial fusion in G1-S phase and blocks cell cycle progression and proliferation. Sci. Signal. 12.

Kutik, S., Rissler, M., Guan, X.L., Guiard, B., Shui, G., Gebert, N., Heacock, P.N., Rehling, P., Dowhan, W., Wenk, M.R., et al. (2008). The translocator maintenance protein Tam41 is required for mitochondrial cardiolipin biosynthesis. J. Cell Biol. 183, 1213-1221. 
Kwong, J.Q. (2017). The mitochondrial calcium uniporter in the heart: energetics and beyond. J. Physiol. 595, 3743-3751.

van der Laan, M., Meinecke, M., Dudek, J., Hutu, D.P., Lind, M., Perschil, I., Guiard, B., Wagner, R., Pfanner, N., and Rehling, P. (2007). Motor-free mitochondrial presequence translocase drives membrane integration of preproteins. Nat. Cell Biol. 9, $1152-1159$.

van der Laan, M., Bohnert, M., Wiedemann, N., and Pfanner, N. (2012). Role of MINOS in mitochondrial membrane architecture and biogenesis. Trends Cell Biol. 22, 185-192.

Laemmli, U.K. (1970). Cleavage of structural proteins during the assembly of the head of bacteriophage T4. Nature 227, 680-685.

Laird, D.M., Parce, J.W., Montgomery, R.I., and Cunningham, C.C. (1986). Effect of phospholipids on the catalytic subunits of the mitochondrial F0.F1-ATPase. J. Biol. Chem. 261, 14851-14856.

Lambert, J.P., Luongo, T.S., Tomar, D., Jadiya, P., Gao, E., Zhang, X., Lucchese, A.M., Kolmetzky, D.W., Shah, N.S., and Elrod, J.W. (2019). MCUB Regulates the Molecular Composition of the Mitochondrial Calcium Uniporter Channel to Limit Mitochondrial Calcium Overload During Stress. Circulation 140, 1720-1733.

Lambert, J.P., Murray, E.K., and Elrod, J.W. (2020). MCUB and mitochondrial calcium uptake - modeling, function, and therapeutic potential. Expert Opin. Ther. Targets 24, 163-169.

Lenaz, G., and Genova, M.L. (2009). Structural and functional organization of the mitochondrial respiratory chain: a dynamic super-assembly. Int. J. Biochem. Cell Biol. 41, 1750-1772.

Li, L., Li, S., Jones, M.K., and Segrest, J.P. (2012). Rotational and hinge dynamics of discoidal high density lipoproteins probed by interchain disulfide bond formation. Biochim. Biophys. Acta 1821, 481-489.

Lill, R., and Mühlenhoff, U. (2008). Maturation of iron-sulfur proteins in eukaryotes: mechanisms, connected processes, and diseases. Annu. Rev. Biochem. 77, 669-700.

Liu, J.C., Liu, J., Holmström, K.M., Menazza, S., Parks, R.J., Fergusson, M.M., Yu, Z.-X., Springer, D.A., Halsey, C., Liu, C., et al. (2016). MICU1 Serves as a Molecular Gatekeeper to Prevent In Vivo Mitochondrial Calcium Overload. Cell Rep. 16, 15611573 .

Logan, C.V., Szabadkai, G., Sharpe, J.A., Parry, D.A., Torelli, S., Childs, A.-M., Kriek, M., Phadke, R., Johnson, C.A., Roberts, N.Y., et al. (2014). Loss-of-function mutations in MICU1 cause a brain and muscle disorder linked to primary alterations in mitochondrial calcium signaling. Nat. Genet. 46, 188-193.

Lu, B., Xu, F.Y., Jiang, Y.J., Choy, P.C., Hatch, G.M., Grunfeld, C., and Feingold, K.R. (2006). Cloning and characterization of a cDNA encoding human cardiolipin synthase (hCLS1). J. Lipid Res. 47, 1140-1145. 
Lutter, M., Fang, M., Luo, X., Nishijima, M., Xie, X., and Wang, X. (2000). Cardiolipin provides specificity for targeting of tBid to mitochondria. Nat. Cell Biol. 2, 754-761.

Maguire, J.J., Tyurina, Y.Y., Mohammadyani, D., Kapralov, A.A., Anthonymuthu, T.S., Qu, F., Amoscato, A.A., Sparvero, L.J., Tyurin, V.A., Planas-Iglesias, J., et al. (2017). Known unknowns of cardiolipin signaling: The best is yet to come. Biochim. Biophys. Acta Mol. Cell Biol. Lipids 1862, 8-24.

Malhotra, A., Edelman-Novemsky, I., Xu, Y., Plesken, H., Ma, J., Schlame, M., and Ren, M. (2009). Role of calcium-independent phospholipase A2 in the pathogenesis of Barth syndrome. Proc. Natl. Acad. Sci. U. S. A. 106, 2337-2341.

Mallilankaraman, K., Cárdenas, C., Doonan, P.J., Chandramoorthy, H.C., Irrinki, K.M., Golenár, T., Csordás, G., Madireddi, P., Yang, J., Müller, M., et al. (2015). MCUR1 is an essential component of mitochondrial $\mathrm{Ca}(2+)$ uptake that regulates cellular metabolism. Nat. Cell Biol. 17, 953.

Mancuso, D.J., Sims, H.F., Han, X., Jenkins, C.M., Guan, S.P., Yang, K., Moon, S.H., Pietka, T., Abumrad, N.A., Schlesinger, P.H., et al. (2007). Genetic ablation of calcium-independent phospholipase A2gamma leads to alterations in mitochondrial lipid metabolism and function resulting in a deficient mitochondrial bioenergetic phenotype. J. Biol. Chem. 282, 34611-34622.

Martin, J., Mahlke, K., and Pfanner, N. (1991). Role of an energized inner membrane in mitochondrial protein import. Delta psi drives the movement of presequences. J. Biol. Chem. 266, 18051-18057.

Martinez-Caballero, S., Grigoriev, S.M., Herrmann, J.M., Campo, M.L., and Kinnally, K.W. (2007). Tim $17 p$ regulates the twin pore structure and voltage gating of the mitochondrial protein import complex TIM23. J. Biol. Chem. 282, 3584-3593.

McKenzie, M., Lazarou, M., Thorburn, D.R., and Ryan, M.T. (2006). Mitochondrial respiratory chain supercomplexes are destabilized in Barth Syndrome patients. J. Mol. Biol. 361, 462-469.

Mejia, E.M., Cole, L.K., and Hatch, G.M. (2014). Cardiolipin metabolism and the role it plays in heart failure and mitochondrial supercomplex formation. Cardiovasc. Hematol. Disord. Drug Targets. 14, 98-106.

Merante, F., Tein, I., Benson, L., and Robinson, B.H. (1994). Maternally inherited hypertrophic cardiomyopathy due to a novel T-to-C transition at nucleotide 9997 in the mitochondrial tRNA(glycine) gene. Am. J. Hum. Genet. 55, 437-446.

Milenkovic, D., Blaza, J.N., Larsson, N.-G., and Hirst, J. (2017). The Enigma of the Respiratory Chain Supercomplex. Cell Metab. 25, 765-776.

Morgenstern, M., Stiller, S.B., Lübbert, P., Peikert, C.D., Dannenmaier, S., Drepper, F., Weill, U., Höß, P., Feuerstein, R., Gebert, M., et al. (2017). Definition of a HighConfidence Mitochondrial Proteome at Quantitative Scale. Cell Rep. 19, 2836-2852. 
Nemani, N., Shanmughapriya, S., and Madesh, M. (2018). Molecular regulation of MCU: Implications in physiology and disease. Cell Calcium 74, 86-93.

Neupert, W., and Herrmann, J.M. (2007). Translocation of proteins into mitochondria. Annu. Rev. Biochem. 76, 723-749.

Ng, D.S. (2005). The role of statins in oxidative stress and cardiovascular disease. Curr. Drug Targets Cardiovasc. Haematol. Disord. 5, 165-175.

Nicolay, K., Rojo, M., Wallimann, T., Demel, R., and Hovius, R. (1990). The role of contact sites between inner and outer mitochondrial membrane in energy transfer. Biochim. Biophys. Acta 1018, 229-233.

Opalińska, M., and Jańska, H. (2018). AAA Proteases: Guardians of Mitochondrial Function and Homeostasis. Cells 7.

Orstavik, K.H., Orstavik, R.E., Naumova, A.K., D’Adamo, P., Gedeon, A., Bolhuis, P.A., Barth, P.G., and Toniolo, D. (1998). X chromosome inactivation in carriers of Barth syndrome. Am. J. Hum. Genet. 63, 1457-1463.

Paillard, M., Csordás, G., Szanda, G., Golenár, T., Debattisti, V., Bartok, A., Wang, N., Moffat, C., Seifert, E.L., Spät, A., et al. (2017). Tissue-Specific Mitochondrial Decoding of Cytoplasmic Ca2+ Signals Is Controlled by the Stoichiometry of MICU1/2 and MCU. Cell Rep. 18, 2291-2300.

Paillard, M., Csordás, G., Huang, K.-T., Várnai, P., Joseph, S.K., and Hajnóczky, G. (2018). MICU1 Interacts with the D-Ring of the MCU Pore to Control Its Ca2+ Flux and Sensitivity to Ru360. Mol. Cell 72, 778-785.e3.

Patron, M., Checchetto, V., Raffaello, A., Teardo, E., Vecellio Reane, D., Mantoan, M., Granatiero, V., Szabò, I., De Stefani, D., and Rizzuto, R. (2014). MICU1 and MICU2 finely tune the mitochondrial $\mathrm{Ca} 2+$ uniporter by exerting opposite effects on MCU activity. Mol. Cell 53, 726-737.

Paupe, V., and Prudent, J. (2018). New insights into the role of mitochondrial calcium homeostasis in cell migration. Biochem. Biophys. Res. Commun. 500, 75-86.

Paupe, V., Prudent, J., Dassa, E.P., Rendon, O.Z., and Shoubridge, E.A. (2015). CCDC90A (MCUR1) is a cytochrome c oxidase assembly factor and not a regulator of the mitochondrial calcium uniporter. Cell Metab. 21, 109-116.

Penna, E., Espino, J., De Stefani, D., and Rizzuto, R. (2018). The MCU complex in cell death. Cell Calcium 69, 73-80.

Petrungaro, C., Zimmermann, K.M., Küttner, V., Fischer, M., Dengjel, J., Bogeski, I., and Riemer, J. (2015). The Ca(2+)-Dependent Release of the Mia40-Induced MICU1MICU2 Dimer from MCU Regulates Mitochondrial $\mathrm{Ca}(2+)$ Uptake. Cell Metab. 22, $721-733$.

Peyta, L., Jarnouen, K., Pinault, M., Guimaraes, C., Pais de Barros, J.-P., Chevalier, S., Dumas, J.-F., Maillot, F., Hatch, G.M., Loyer, P., et al. (2016). Reduced 
cardiolipin content decreases respiratory chain capacities and increases ATP synthesis yield in the human HepaRG cells. Biochim. Biophys. Acta 1857, 443-453.

Phillips, C.B., Tsai, C.-W., and Tsai, M.-F. (2019). The conserved aspartate ring of MCU mediates MICU1 binding and regulation in the mitochondrial calcium uniporter complex. ELife 8 .

Raffaello, A., De Stefani, D., Sabbadin, D., Teardo, E., Merli, G., Picard, A., Checchetto, V., Moro, S., Szabò, I., and Rizzuto, R. (2013). The mitochondrial calcium uniporter is a multimer that can include a dominant-negative pore-forming subunit. EMBO J. 32, 2362-2376.

Richter-Dennerlein, R., Korwitz, A., Haag, M., Tatsuta, T., Dargazanli, S., Baker, M., Decker, T., Lamkemeyer, T., Rugarli, E.I., and Langer, T. (2014). DNAJC19, a mitochondrial cochaperone associated with cardiomyopathy, forms a complex with prohibitins to regulate cardiolipin remodeling. Cell Metab. 20, 158-171.

Roise, D., Horvath, S.J., Tomich, J.M., Richards, J.H., and Schatz, G. (1986). A chemically synthesized pre-sequence of an imported mitochondrial protein can form an amphiphilic helix and perturb natural and artificial phospholipid bilayers. EMBO J. $5,1327-1334$.

Ronvelia, D., Greenwood, J., Platt, J., Hakim, S., and Zaragoza, M.V. (2012). Intrafamilial variability for novel TAZ gene mutation: Barth syndrome with dilated cardiomyopathy and heart failure in an infant and left ventricular noncompaction in his great-uncle. Mol. Genet. Metab. 107, 428-432.

Saitoh, T., Igura, M., Obita, T., Ose, T., Kojima, R., Maenaka, K., Endo, T., and Kohda, D. (2007). Tom 20 recognizes mitochondrial presequences through dynamic equilibrium among multiple bound states. EMBO J. 26, 4777-4787.

Saric, A., Andreau, K., Armand, A.-S., Møller, I.M., and Petit, P.X. (2015). Barth Syndrome: From Mitochondrial Dysfunctions Associated with Aberrant Production of Reactive Oxygen Species to Pluripotent Stem Cell Studies. Front. Genet. 6, 359.

Schägger, H. (2002). Respiratory chain supercomplexes of mitochondria and bacteria. Biochim. Biophys. Acta 1555, 154-159.

Schlame, M., and Haldar, D. (1993). Cardiolipin is synthesized on the matrix side of the inner membrane in rat liver mitochondria. J. Biol. Chem. 268, 74-79.

Schlame, M., and Ren, M. (2006). Barth syndrome, a human disorder of cardiolipin metabolism. FEBS Lett. 580, 5450-5455.

Schlame, M., Kelley, R.I., Feigenbaum, A., Towbin, J.A., Heerdt, P.M., Schieble, T., Wanders, R.J.A., DiMauro, S., and Blanck, T.J.J. (2003). Phospholipid abnormalities in children with Barth syndrome. J. Am. Coll. Cardiol. 42, 1994-1999.

Schlame, M., Ren, M., Xu, Y., Greenberg, M.L., and Haller, I. (2005). Molecular symmetry in mitochondrial cardiolipins. Chem. Phys. Lipids 138, 38-49. 
Schulz, C., Lytovchenko, O., Melin, J., Chacinska, A., Guiard, B., Neumann, P., Ficner, R., Jahn, O., Schmidt, B., and Rehling, P. (2011). Tim50's presequence receptor domain is essential for signal driven transport across the TIM23 complex. J. Cell Biol. 195, 643-656.

Schwall, C.T., Greenwood, V.L., and Alder, N.N. (2012). The stability and activity of respiratory Complex II is cardiolipin-dependent. Biochim. Biophys. Acta 1817, 15881596.

Sedlák, E., and Robinson, N.C. (1999). Phospholipase A(2) digestion of cardiolipin bound to bovine cytochrome c oxidase alters both activity and quaternary structure. Biochemistry 38, 14966-14972.

Sedlák, E., Panda, M., Dale, M.P., Weintraub, S.T., and Robinson, N.C. (2006). Photolabeling of cardiolipin binding subunits within bovine heart cytochrome $\mathrm{c}$ oxidase. Biochemistry 45, 746-754.

Serricchio, M., Vissa, A., Kim, P.K., Yip, C.M., and McQuibban, G.A. (2018). Cardiolipin synthesizing enzymes form a complex that interacts with cardiolipindependent membrane organizing proteins. Biochim. Biophys. Acta Mol. Cell Biol. Lipids $1863,447-457$.

Shariff, K., Ghosal, S., and Matouschek, A. (2004). The force exerted by the membrane potential during protein import into the mitochondrial matrix. Biophys. J. $86,3647-3652$.

Sharpley, M.S., and Hirst, J. (2006). The inhibition of mitochondrial complex I (NADH:ubiquinone oxidoreductase) by Zn2+. J. Biol. Chem. 281, 34803-34809.

Shen, H., and Dowhan, W. (1998). Regulation of phosphatidylglycerophosphate synthase levels in Saccharomyces cerevisiae. J. Biol. Chem. 273, 11638-11642.

Shen, H., Heacock, P.N., Clancey, C.J., and Dowhan, W. (1996). The CDS1 gene encoding CDP-diacylglycerol synthase in Saccharomyces cerevisiae is essential for cell growth. J. Biol. Chem. 271, 789-795.

Soustek, M.S., Falk, D.J., Mah, C.S., Toth, M.J., Schlame, M., Lewin, A.S., and Byrne, B.J. (2011). Characterization of a transgenic short hairpin RNA-induced murine model of Tafazzin deficiency. Hum. Gene Ther. 22, 865-871.

Spencer, C.T., Byrne, B.J., Gewitz, M.H., Wechsler, S.B., Kao, A.C., Gerstenfeld, E.P., Merliss, A.D., Carboni, M.P., and Bryant, R.M. (2005). Ventricular arrhythmia in the X-linked cardiomyopathy Barth syndrome. Pediatr. Cardiol. 26, 632-637.

Spencer, C.T., Bryant, R.M., Day, J., Gonzalez, I.L., Colan, S.D., Thompson, W.R., Berthy, J., Redfearn, S.P., and Byrne, B.J. (2006). Cardiac and clinical phenotype in Barth syndrome. Pediatrics 118, e337-346.

Sproule, D.M., and Kaufmann, P. (2008). Mitochondrial encephalopathy, lactic acidosis, and strokelike episodes: basic concepts, clinical phenotype, and therapeutic management of MELAS syndrome. Ann. N. Y. Acad. Sci. 1142, 133-158. 
Szeto, H.H. (2014). First-in-class cardiolipin-protective compound as a therapeutic agent to restore mitochondrial bioenergetics. Br. J. Pharmacol. 171, 2029-2050.

Tamura, Y., and Endo, T. (2013). Unveiling the last missing link of the cardiolipin synthetic pathway in mitochondria. Aging 5, 392-393.

Tamura, Y., Harada, Y., Yamano, K., Watanabe, K., Ishikawa, D., Ohshima, C., Nishikawa, S., Yamamoto, H., and Endo, T. (2006). Identification of Tam41 maintaining integrity of the TIM23 protein translocator complex in mitochondria. J. Cell Biol. 174, 631-637.

Tamura, Y., Endo, T., Iijima, M., and Sesaki, H. (2009). Ups1p and Ups2p antagonistically regulate cardiolipin metabolism in mitochondria. J. Cell Biol. 185, $1029-1045$.

Tatsuta, T., and Langer, T. (2017). Intramitochondrial phospholipid trafficking. Biochim. Biophys. Acta Mol. Cell Biol. Lipids 1862, 81-89.

Taylor, W.A., and Hatch, G.M. (2009). Identification of the human mitochondrial linoleoyl-coenzyme A monolysocardiolipin acyltransferase (MLCL AT-1). J. Biol. Chem. 284, 30360-30371.

Tohyama, S., Hattori, F., Sano, M., Hishiki, T., Nagahata, Y., Matsuura, T., Hashimoto, H., Suzuki, T., Yamashita, H., Satoh, Y., et al. (2013). Distinct metabolic flow enables large-scale purification of mouse and human pluripotent stem cellderived cardiomyocytes. Cell Stem Cell 12, 127-137.

Tomar, D., Dong, Z., Shanmughapriya, S., Koch, D.A., Thomas, T., Hoffman, N.E., Timbalia, S.A., Goldman, S.J., Breves, S.L., Corbally, D.P., et al. (2016). MCUR1 Is a Scaffold Factor for the MCU Complex Function and Promotes Mitochondrial Bioenergetics. Cell Rep. 15, 1673-1685.

Tsai, C.-W., Wu, Y., Pao, P.-C., Phillips, C.B., Williams, C., Miller, C., Ranaghan, M., and Tsai, M.-F. (2017). Proteolytic control of the mitochondrial calcium uniporter complex. Proc. Natl. Acad. Sci. U. S. A. 114, 4388-4393.

Tsai, M.-F., Phillips, C.B., Ranaghan, M., Tsai, C.-W., Wu, Y., Willliams, C., and Miller, C. (2016a). Dual functions of a small regulatory subunit in the mitochondrial calcium uniporter complex. ELife 5.

Tsai, M.-F., Phillips, C.B., Ranaghan, M., Tsai, C.-W., Wu, Y., Willliams, C., and Miller, C. (2016b). Dual functions of a small regulatory subunit in the mitochondrial calcium uniporter complex. ELife 5.

Tyurina, Y.Y., Domingues, R.M., Tyurin, V.A., Maciel, E., Domingues, P., Amoscato, A.A., Bayir, H., and Kagan, V.E. (2014). Characterization of cardiolipins and their oxidation products by LC-MS analysis. Chem. Phys. Lipids 179, 3-10.

Vais, H., Tanis, J.E., Müller, M., Payne, R., Mallilankaraman, K., and Foskett, J.K. (2015). MCUR1, CCDC90A, Is a Regulator of the Mitochondrial Calcium Uniporter. Cell Metab. 22, 533-535. 
Valianpour, F., Wanders, R.J.A., Overmars, H., Vreken, P., Van Gennip, A.H., Baas, F., Plecko, B., Santer, R., Becker, K., and Barth, P.G. (2002). Cardiolipin deficiency in X-linked cardioskeletal myopathy and neutropenia (Barth syndrome, MIM 302060): a study in cultured skin fibroblasts. J. Pediatr. 141, 729-733.

Vecellio Reane, D., Vallese, F., Checchetto, V., Acquasaliente, L., Butera, G., De Filippis, V., Szabò, I., Zanotti, G., Rizzuto, R., and Raffaello, A. (2016). A MICU1 Splice Variant Confers High Sensitivity to the Mitochondrial Ca2+ Uptake Machinery of Skeletal Muscle. Mol. Cell 64, 760-773.

Vögtle, F.-N., Burkhart, J.M., Gonczarowska-Jorge, H., Kücükköse, C., Taskin, A.A., Kopczynski, D., Ahrends, R., Mossmann, D., Sickmann, A., Zahedi, R.P., et al. (2017). Landscape of submitochondrial protein distribution. Nat. Commun. 8, 290.

Wallace, D.J., Meyer zum Alten Borgloh, S., Astori, S., Yang, Y., Bausen, M., Kügler, S., Palmer, A.E., Tsien, R.Y., Sprengel, R., Kerr, J.N.D., et al. (2008). Singlespike detection in vitro and in vivo with a genetic Ca2+ sensor. Nat. Methods 5, 797804.

Wang, G., McCain, M.L., Yang, L., He, A., Pasqualini, F.S., Agarwal, A., Yuan, H., Jiang, D., Zhang, D., Zangi, L., et al. (2014). Modeling the mitochondrial cardiomyopathy of Barth syndrome with induced pluripotent stem cell and heart-onchip technologies. Nat. Med. 20, 616-623.

Wang, Y., Nguyen, N.X., She, J., Zeng, W., Yang, Y., Bai, X.-C., and Jiang, Y. (2019). Structural Mechanism of EMRE-Dependent Gating of the Human Mitochondrial Calcium Uniporter. Cell 177, 1252-1261.e13.

Weber, T.A., Koob, S., Heide, H., Wittig, I., Head, B., van der Bliek, A., Brandt, U., Mittelbronn, M., and Reichert, A.S. (2013). APOOL is a cardiolipin-binding constituent of the Mitofilin/MINOS protein complex determining cristae morphology in mammalian mitochondria. PloS One 8 , e63683.

Wenz, T., Hielscher, R., Hellwig, P., Schägger, H., Richers, S., and Hunte, C. (2009). Role of phospholipids in respiratory cytochrome bc(1) complex catalysis and supercomplex formation. Biochim. Biophys. Acta 1787, 609-616.

Wescott, A.P., Kao, J.P.Y., Lederer, W.J., and Boyman, L. (2019). Voltage-energized Calcium-sensitive ATP Production by Mitochondria. Nat. Metab. 1, 975-984.

Wettmarshausen, J., Goh, V., Huang, K.-T., Arduino, D.M., Tripathi, U., Leimpek, A., Cheng, Y., Pittis, A.A., Gabaldón, T., Mokranjac, D., et al. (2018). MICU1 Confers Protection from MCU-Dependent Manganese Toxicity. Cell Rep. 25, 14251435.e7.

Xiao, J., Engel, J.L., Zhang, J., Chen, M.J., Manning, G., and Dixon, J.E. (2011). Structural and functional analysis of PTPMT1, a phosphatase required for cardiolipin synthesis. Proc. Natl. Acad. Sci. U. S. A. 108, 11860-11865.

Xu, Y., and Schlame, M. (2014). The turnover of glycerol and acyl moieties of cardiolipin. Chem. Phys. Lipids 179, 17-24. 
Xu, Y., Phoon, C.K.L., Berno, B., D’Souza, K., Hoedt, E., Zhang, G., Neubert, T.A., Epand, R.M., Ren, M., and Schlame, M. (2016). Loss of protein association causes cardiolipin degradation in Barth syndrome. Nat. Chem. Biol. 12, 641-647.

Yamamoto, H., Esaki, M., Kanamori, T., Tamura, Y., Nishikawa, S. ichi, and Endo, T. (2002). Tim50 is a subunit of the TIM23 complex that links protein translocation across the outer and inner mitochondrial membranes. Cell 111, 519-528.

Yamamoto, T., Yamagoshi, R., Harada, K., Kawano, M., Minami, N., Ido, Y., Kuwahara, K., Fujita, A., Ozono, M., Watanabe, A., et al. (2016). Analysis of the structure and function of EMRE in a yeast expression system. Biochim. Biophys. Acta $1857,831-839$.

Yamano, K., Yatsukawa, Y.-I., Esaki, M., Hobbs, A.E.A., Jensen, R.E., and Endo, T. (2008). Tom 20 and Tom 22 share the common signal recognition pathway in mitochondrial protein import. J. Biol. Chem. 283, 3799-3807.

Yoda, E., Hachisu, K., Taketomi, Y., Yoshida, K., Nakamura, M., Ikeda, K., Taguchi, R., Nakatani, Y., Kuwata, H., Murakami, M., et al. (2010). Mitochondrial dysfunction and reduced prostaglandin synthesis in skeletal muscle of Group VIB Ca2+independent phospholipase A2gamma-deficient mice. J. Lipid Res. 51, 3003-3015.

Yoo, J., Wu, M., Yin, Y., Herzik, M.A., Lander, G.C., and Lee, S.-Y. (2018). CryoEM structure of a mitochondrial calcium uniporter. Science 361, 506-511.

Youle, R.J., and van der Bliek, A.M. (2012). Mitochondrial fission, fusion, and stress. Science 337, 1062-1065.

Zhang, J., Guan, Z., Murphy, A.N., Wiley, S.E., Perkins, G.A., Worby, C.A., Engel, J.L., Heacock, P., Nguyen, O.K., Wang, J.H., et al. (2011). Mitochondrial phosphatase PTPMT1 is essential for cardiolipin biosynthesis. Cell Metab. 13, 690700 .

Zhang, L., Li, Z., Quinn, B., Yu, L., and Yu, C.-A. (2002). Nonoxidizable ubiquinol derivatives that are suitable for the study of the ubiquinol oxidation site in the cytochrome bc1 complex. Biochim. Biophys. Acta 1556, 226-232.

Zheng, X., Chen, M., Meng, X., Chu, X., Cai, C., and Zou, F. (2017). Phosphorylation of dynamin-related protein 1 at Ser616 regulates mitochondrial fission and is involved in mitochondrial calcium uniporter-mediated neutrophil polarization and chemotaxis. Mol. Immunol. 87, 23-32.

Zhong, Q., and Greenberg, M.L. (2003). Regulation of phosphatidylglycerophosphate synthase by inositol in Saccharomyces cerevisiae is not at the level of PGS1 mRNA abundance. J. Biol. Chem. 278, 33978-33984.

Mitochondrial contact sites. Lipid composition and dynamics. - PubMed - NCBI.

Lipids in membrane protein structures. - PubMed - NCBI.

The non-glycosylated isoform of MIC26 is a constituent of the mammalian MICOS complex and promotes formation of crista junctions. - PubMed - NCBI. 
Architecture of the mitochondrial calcium uniporter. - PubMed - NCBI.

\section{Acknowledgements}

First, I would like to remark my gratefulness to my first supervisor Peter Rehling, who streamlined me and gave me the opportunity to work on this challenging and interesting project. I have benefited from his great supervision and I want to thank Peter for all the helpful discussions to develop this project and the guidance trough all the steps of my work. Peter had a very motivating leadership style to conduct me as a PhD student.

I want to thank Doerthe Katschinski for all the helpful advices in the thesis committee meetings. Beside this project, Doerthe Katschinski gave me the opportunity to collaborate with her lab members on aspects of MCU in hypoxic environments. I profited from her expertise in physiology, calcium metabolism and hypoxia. I could follow my ideas on questions of MCU complex organization in hypoxia in her lab and I could give back my expertise in BN-PAGE analysis.

I benefited from the great expertise in molecular biochemistry of Blanche Schwappach, who gave me crucial ideas and suggestions for experiments, which found their way into my thesis. In general, I really appreciate, that we had very productive and innovative thesis committee meetings, which gave me new helpful directions in my projects.

I want to say my gratitude to Detlef Doenecke, Henning Urlaub and Ralf Kehlenbach for serving on my extended thesis examination board.

Jan Dudek supervised me on a daily bases before he left to Würzburg. We were continuously in contact and we met regularly and had productive discussions. I want to thank him for his positive and motivating approach on supervising me and his 
suggestions, which always gave me a new view on my projects. To be honest, I was really looking forward on seeing Jan again on the late Friday afternoons we met.

I want to show my appreciation to all members of the Department of Cellular Biochemistry. In particular, I want to thank Arpita and Abhishek for supervision and inspiring discussion on my work.

I benefited from the expertise in calcium metabolism and calcium measurements by Ivan Bogeski and Zurine Bonilla del Rio. I really enjoyed the time and discussions with you.

\section{Curriculum Vitae}

Personal Information

Name:

Magnus Hartmann

Adress:

Von-Ossietzky-Straße 42, 37085 Göttingen; Germany

Nationality:

German

Date of birth:

01/01/1989

Gender:

Male

\section{Education}

$10 / 2012-12 / 2014$

Hannover Medical School (MHH) and University of Oxford

Studying Biochemistry (Master of Science)

Final mark: 1.2

10/2009 - 09/2012 Gottfried Leibniz University Hannover (LUH) and MHH

Studying Biochemistry (Bachelor of Science)

Final mark: 1.7

$08 / 2001-07 / 2008$

Michelsenschule Hildesheim (grammar school)

Final mark of A-levels: 1.7

Main subjects in A-level: math, chemistry, biology

Research experiences

04-2016- 2020

Department of Cellular Biochemistry, UMG

Research assistant, PhD student

"Characterization of Mitochondrial Calcium Uniporter in Barth Syndrome Models" 
$02-12 / 2015$

$08-12 / 2014$

$01-07 / 2014$

$10-12 / 2013$

$07-08 / 2012$
German Institute of Human Nutrition Potsdam-Rehbruecke

Department of Experimental Diabetology, Research Assistant

"Influence of scaffold proteins at the Golgi apparatus on protein sorting to the lipid droplet"

Methods: Cell culture and differentiation (3T3-L1 adipocytes, Caco-2 cells), electroporation, immuncytochemistry, live-cell imaging, confocal fluorescence microscopy, qPCR, cell fractionation/ultracentrifugation, Western Blotting supervised by Prof Annette Schuermann

University of Oxford - Department of Physiology, Anatomy and Genetics ERASMUS+ funded internship abroad:

"Investigation the role of acetyl-CoA carboxylase-1 and de novo lipogenesis in the control of insulin secretion and beta cell size"

Methods: Cell culture with clonal beta cells (MIN6), Western Blotting supervised by PhD James Cantley (see references)

Institute of Clinical Biochemistry, MHH; Master's thesis on the topic: "Investigation of lipid droplet associated proteins regarding lipotoxicity in insulinproducing cells"

Methods: Cell culture with clonal beta cells (RINm5F, INS-1E), qPCR, Western Blotting, fluorescence microscopy, plasmid cloning, Oil-red-O staining, Cell viability assays (MTT), Lentiviral transfection of ShRNA/RNAi

\section{Final mark: 1.0}

supervised by PD Dr Matthias Elsner (see references), Prof Lenzen

Institute of Neuroanatomy, MHH; voluntary practical course:

"EKAR - a FRET-based sensor of ERK activity in neuronal cells"

Methods: Cell culture, Western Blotting, epi/konfocal fluorescence microscopy

supervised by Prof Peter Claus (2. Examiner of master's thesis, see references)

Institute of Clinical Biochemistry, MHH; Bachelor's thesis on the topic:

"Expression analysis at important T-cell genes in the LEW.1AR1-iddm rat - an animal model for human Type 1 Diabetes Mellitus"

Methods: RNA isolation, qPCR 
Final mark: 1.0;

supervised by Dr Tanja Arndt (see references), Prof Lenzen

\section{Publications}

The role of mitochondrial cardiolipin in heart function and its implication in cardiac disease.

Dudek J, Hartmann M, Rehling P.

Biochim Biophys Acta Mol Basis Dis. 2019 Apr 1;1865(4):810-821. doi: 10.1016/j.bbadis.2018.08.025. Epub 2018 Aug 26. Review.

Defective Mitochondrial Cardiolipin Remodeling Dampens HIF-1 $\alpha$ Expression in Hypoxia. Chowdhury A, Aich A, Jain G, Wozny K, Lüchtenborg C, Hartmann M, Bernhard O, Balleiniger M, Alfar EA, Zieseniss A, Toischer K, Guan K, Rizzoli SO, Brügger B, Fischer A, Katschinski DM, Rehling P, Dudek J. Cell Rep. 2018 Oct 16;25(3):561-570.e6. doi: 10.1016/j.celrep.2018.09.057.

The role of lipid droplet formation in the protection of unsaturated fatty acids against palmitic acid induced lipotoxicity to rat insulin-producing cells.

Plötz T, Hartmann M, Lenzen S, Elsner M.

Nutr Metab (Lond). 2016 Feb 25;13:16. doi: 10.1186/s12986-016-0076-z. eCollection 2016.

\section{Teaching skills}

$04 / 2016$ to date

Department of Cellular Biochemistry, University Medical Center Göttingen, Germany

Giving seminar lectures and supervision of practical courses in biochemistry for

medical students

$04 / 2017$ to date

Department of Cellular Biochemistry, University Medical Center Göttingen, Germany

Giving method courses (Blue-native PAGE analysis) for PhD student of the

GGNB program (Göttinger Graduiertenzentrum für Neurowisenschaften,

Biophysik und Molekulare Biocwissenschaften)

\section{Skills}

Selection of my molecular biological skills:

FELASA-B course (practical and theoretical part),

Certificate for handling radioactive isotopes (Fachkundegruppe S4.2)

Certificate for gene technology, biosafety and biosecurity

(§15 Abs. 2 Satz 1 Nr.3 GenTSV)

Cell culture, qPCR, Western Blotting, cell fractionation/ultracentrifugation, confocal fluorescence microscopy, FACS

EDP: $\quad$ Microsoft Office Word, Excel, Powerpoint

Languages: German - native language

English - fluently; from 5th to 12 th class,

Research internship at University of Oxford in August-October 2014, supported by James Cantley, ERASMUS+ funded 
Latin - Latin proficiency certificate within A-levels (2008)

Successful participation in a competition of old languages in Lower Saxony

Finaly mark of skilled work: 12 of 15 points;

corrected by Union of classical philologists Lower Saxony, Germany

Spanish - A1 level course at University of Göttingen

Driving licences: $\mathrm{B}, \mathrm{BE}$ and $\mathrm{T}$

First aid: $\quad$ Education in "first aid at the scene of the accident" 2018

\section{Personal interests and activities}

Engine powered flight: Light aircraft pilot licence (LAPL(A)) at Aeroclub Hildesheim/Hannover Justification to fly single-engine airplanes up to $2000 \mathrm{~kg}$ in Europe (since 2009)

Private investing, Active member of Akademischer Börsenverein Göttingen eV (abvg) Stock exchange activities Management of familar asset portfolio following long-term buy and hold strategies

Annual Cycling tours: Pilgrimage on the French and Spanish Camino de Santiago from Paris to Santiago de Compostella, Alpine cross from Munich to Verona,

Italy

Jogging: Annual participation in several half marathons in Hannover, Potsdam and Berlin (since 2014)

Playing piano: $\quad$ Piano education from 09/2003 - 09/2009

Accompaniment of children's choir of Michelsenschule Hildesheim (2006)

Ballroom dance: Dancesport activity (since 2003)

Several successful participations in German championships

\section{References}

PhD James Cantley (beta cell function, obesity and type 2 diabetes)

University of Oxford - Department of Physiology, Anatomy and Genetics

james.cantley@dpag.ox.ac.uk

PD Dr Matthias Elsner (Lipotoxicity in beta cells, obesity, T2DM);

Institute of Clinical Biochemistry, MHH; Elsner.Matthias@mh-hannover.de

Prof Peter Claus (Neuroanatomy, SMA/ALS); Institute of Neuroanatomy, MHH

Claus.Peter@mh-hannover.de 
Prof Annette Schuermann (Genetic and pathophysiological basis of insulin resistance and type 2 diabetes)

German Institute of Human Nutrition Potsdam-Rehbruecke - Department of Experimental Diabetology

Dr Tanja Arndt (Genetical backrounds regarding Type I Diabetes Mellitus);

Institute of Clinical Biochemistry, MHH; arndt.tanja@mh-hannover.de 